\title{
Resource Allocation Under Delay-Guarantee Constraints for Heterogeneous Visible-Light and RF Femtocell
}

\author{
Fan Jin, Rong Zhang, Member, IEEE, and Lajos Hanzo, Fellow, IEEE
}

\begin{abstract}
The resource-allocation (RA) problems of mobile terminals (MTs) are investigated in a heterogeneous wireless network, where both a visible light communication system and an RF femtocell system are deployed in a room. We consider diverse quality-of-service requirements in terms of the data rate, fairness, and statistical delay requirements. Inspired by the concept of effective capacity, we formulate our optimization problems applying $\alpha$-proportional fairness while satisfying specific statistical delay constraints. Two types of MTs, namely, multihoming MTs and multimode MTs, are considered, where multihoming MTs have the capability of aggregating resources from different networks, whereas the multimode MTs always select a single network for their connection. Our optimization procedure solves the RA probability problem for multihoming MTs with the aid of a decentralized algorithm. By contrast, our optimization problem involves both network selection and RA probability optimization for multimode MTs, which may be regarded as a mixed-integer nonlinear problem. Since this problem is computationally intractable, a suboptimal decentralized method is proposed for solving it. Simulation results are also presented for clarifying the performance of the proposed algorithm. It is shown that the multimode MTs are capable of achieving similar performance to that of the multihoming MTs when the statistical delay requirements are loose. However, as expected, the multihoming MTs attain a better performance when we tighten the delay requirements.
\end{abstract}

Index Terms-Resource allocation, heterogeneous networks, VLC system, femtocell system, effective capacity.

\section{INTRODUCTION}

$\mathbf{N}$ EXT-GENERATION wireless networks are expected to be heterogeneous, integrating diverse radio access technologies (RAT) such as UMTS Terrestrial Radio Access Networks (UTRAN), GSM/EDGE Radio Access and Network (GERAN) [1], Wireless Local Area Network (WLAN) as well as millimeter-wave and possibly Visible Light Communications (VLC) networks [2], [3]. All the above networks have a coverage area overlapping with one another, hence forming a

Manuscript received February 12, 2014; revised July 17, 2014 and September 24, 2014; accepted October 10, 2014. This work was supported in part by the European Research Council under the Advanced Fellow Grant, by the RC-U.K. under the auspices of the India-U.K. Advanced Technology Centre, and by the EU's Concerto Project. The associate editor coordinating the review of this paper and approving it for publication was S. Valaee.

The authors are with the School of Electronics and Computer Science, University of Southampton, Southampton SO17 1BJ, U.K. (e-mail: fj1g10@ecs. soton.ac.uk; rz@ecs.soton.ac.uk; lh@ecs.soton.ac.uk).

Color versions of one or more of the figures in this paper are available online at http://ieeexplore.ieee.org.

Digital Object Identifier 10.1109/TWC.2014.2363451 hybrid network for wireless access, which is typically termed as a heterogeneous network (HetNet). A heterogeneous cellular system may consist of regular macro-cell base stations (BS) that typically transmit at a high power level and overlaid pico-cells, femtocells as well as relays, which transmit at substantially lower power levels [4].

Indoor cells may also form part of a HetNet. Femtocells have the potential of providing high-quality network access for indoor users at a low cost [5], [6]. As another recent addition to the wireless landscape, optical wireless systems using visible light exhibit several advantages, including license-free operation, immunity to electro-magnetic interference, network security and a high bandwidth potential [7]. The optical access point is referred to as an attocell in [3] However, the performance of VLC systems is degraded in the absence of line-ofsight (LOS) propagation. Hence we may combine VLC with a complementary network for providing a seamless data service. An optical attocell not only improves indoor coverage, it is able to enhance the capacity of the RF wireless networks, since it does not generate any additional interference. In this paper, we consider an indoor scenario, where a VLC system is combined with a classic RF femtocell system [8] and investigate the associated resource allocation (RA) problems.

For a HetNet, an important component of the integrated architecture is its radio resource management (RRM) mechanism. To access the Internet through a HetNet, the terminals such as laptops and cellphones are usually equipped with multiple wireless access network interfaces. There are two basic types of terminals. The conventional multi-mode mobile terminals are unable to support IP mobility or multi-homing. Hence, these multi-mode terminals typically opt for the specific network providing the best radio-coverage. By contrast, the family of more sophisticated terminals is equipped with IP-based mobility and multi-homing functionalities, which have the capability of aggregating radio resources from different networks. Numerous contributions have studied the RA problem in a HetNet scenario [9]-[12]. Multi-homing RA mechanisms are studied in [9], which allocate the radio bandwidth to different tele-traffic types based on the specific utility of the service supported. However, this required a central resource manager for controlling the RA process. On the other hand, the authors of [10]-[12] propose a distributed algorithm for solving the RA problem. However, these contributions only focus on maximizing the data rate, while the quality-of-service (QoS) requirements formulated in terms of the delay requirements of mobile terminals were not 
considered in these contributions. Furthermore, these contributions considered a pure RF HetNet model, while our paper considered the RA problem of a combined VLC and RF Femtocell HetNet.

Future broadband wireless networks are expected to support a wide variety of communication services having diverse QoS requirements. Applications such as voice transmission as well as real-time lip-synchronized video streaming are delaysensitive and they require a minimum guaranteed throughput. On the other hand, applications such as file transfer and email services are relatively delay-tolerant. As a result, it is important to consider the delay as a performance metrics in addition to the classic Physical (PHY) layer performance metric in crosslayer optimization. Diverse approaches may be conceived for delay-aware resource control in wireless networks [13]-[19]. For example, the average delay-constraint may be converted into an equivalent average rate-constraint using the large deviation theory approach of [13]-[17]. In order to satisfy the QoS requirements, we apply the effective capacity [13] based approach for deriving the optimal RA algorithm, which guarantees meeting the statistical delay target of HetNets.

\section{A. Main Contributions}

In this paper, we address the optimal RA in the DL of a HetNet, while meeting both the bit rate and statistical delay targets of delay-sensitive traffic. The contributions of this paper are summarized as follows:

- We consider an indoor scenario, where a combined VLC attocell and RF femtocell system is employed for providing indoor coverage. For a given position of the mobile terminal (MT), the received optical power of a MT is constituted by the sum of the direct LOS optical power and of the first reflected optical power. Hence the resultant VLC channel has two different rates, a higher one for the non-blocked LOS channel-scenario, and a reduced rate in the blocked LOS channel-scenario, when only the reflected ray is received. The RF channel is modeled as a classic Rayleigh fading channel. We investigate the VLC vs. RF activation and RA problem in this HetNet scenario.

- A limited-delay RA problem is formulated for the indoor HetNet considered. In contrast to the RA solutions of [11], [12], [15], [16], we apply the effective capacity approach of [13] for converting the statistical delay constraints into equivalent average rate constraints. Furthermore, we formulate our fairness-problem as an $\alpha$-proportional fairness utility function, as defined in [20]-[23].

- Our RA problem is formulated as a non-linear programming (NLP) problem for the multi-homing MTs. We show mathematically that this NLP problem is concave with respect to the RA probability matrix $\boldsymbol{\beta}$, which hence can be solved by convex optimization techniques, such as the barrier method of [24]. A distributed algorithm using the dual decomposition approach of [25] is proposed for RA in HetNets. It is demonstrated that this distributed algorithm approaches the optimal solution within a low number of iterations, where the optimal solution is that found by a centralized controller.
TABLE I

The Main Symbol Notations

\begin{tabular}{|c|c|}
\hline \multicolumn{2}{|r|}{ Symbol definition } \\
\hline $\mathcal{M}$ & The network set \\
\hline $\mathcal{N}$ & The mobile terminal (MT) set \\
\hline$x_{m, n}$ & The network assignment index from network $m$ to MT $n$ \\
\hline$\beta_{m, n}$ & The instantaneous probability that network $m$ transmits to MT $n$ \\
\hline$r_{m, n}$ & The instantaneous transmission bit rate \\
\hline$\psi_{i r}$ & Angle of irradiation of LED lights \\
\hline$\psi_{\text {in }}$ & Angle of incidence \\
\hline$D_{d}$ & Distance between a transmitter and a receiver \\
\hline$l$ & The order of Lambertian emission \\
\hline$\phi_{1 / 2}$ & The semi-angle at half illuminant of an LED \\
\hline$A$ & The physical area of the detector in a PD \\
\hline$T_{s}$ & The gain of an optical filter \\
\hline$g$ & The gain of an optical concentrator \\
\hline$\Psi_{c}$ & The width of the field of vision at a receiver \\
\hline$\kappa$ & A random variable with the Bernoulli distribution \\
\hline$P_{t}$ & The transmitted optical power \\
\hline$P_{r}$ & The received optical power \\
\hline$D_{1}$ & The distance between an LED light and a reflecting surface \\
\hline$D_{2}$ & The distance between a reflective point and a receiver \\
\hline$\rho$ & The reflectance factor \\
\hline$d A_{\text {wall }}$ & Reflective area \\
\hline$\omega_{1}$ & The angle of irradiance to a reflective point \\
\hline & The angle of irradiance to a MT's receiver \\
\hline$\sigma_{\text {shot }}^{2}$ & The variance of the short noise \\
\hline$\sigma_{\text {thermal }}^{2}$ & The variance of the thermal noise \\
\hline$q$ & The electronic charge \\
\hline & The detector's responsivity \\
\hline$B$ & The equivalent noise bandwidth \\
\hline$I_{b g}$ & The background current \\
\hline$\varrho$ & The Boltzmann's constant \\
\hline$T_{K}$ & The absolute temperature \\
\hline$G$ & The open-loop voltage gain \\
\hline$g_{m}$ & The FET transconductance \\
\hline$Z$ & The number of constellation points \\
\hline$\iota$ & The path-loss exponent \\
\hline$P_{f}$ & The transmitted RF power \\
\hline$\Pi$ & The recived SNR discrepancy \\
\hline$B_{f}$ & The bandwidth of the RF femtocell \\
\hline$f_{c}$ & RF carrier frequency \\
\hline$h$ & RF Rayleigh-distributed channel gain \\
\hline$\sigma^{2}$ & The variance of the AWGN \\
\hline$\theta$ & Delay-rated QoS statistical paramenter \\
\hline $\mathcal{R}_{1, n}$ & The achievable rate with VLC LOS reception \\
\hline $\mathcal{R}_{2, n}$ & The achievable rate without VLC LOS reception \\
\hline$\lambda$ & The Lagrange multiplier \\
\hline$\mu$ & The Lagrange multiplier \\
\hline$\xi$ & The step-size in the iteration algorithm \\
\hline
\end{tabular}

- We also demonstrate that the RA problem is formulated as a mixed-integer non-linear programming (MINLP) problem for multi-mode MTs, which is mathematically intractable. In order to make the problem more tractable, a relaxation of the integer variables is introduced. Then, we formally prove that the relaxed problem is concave with respect to both the relaxed network selection matrix $\boldsymbol{x}$ and to the RA probability matrix $\boldsymbol{\beta}$. Finally, a distributed algorithm is conceived using the dual decomposition approach for RA in our HetNet for the specific family of MTs having a multi-mode capability, but no multi-homing facility.

The paper is organized as follows. In Section II, we describe our system model, including both the VLC and the RF femtocell system. The formulation of our RA problems for both multi-homing MTs and multi-mode MTs is described in Section III. The proposed distributed algorithms are outlined in Section IV. Finally, our results are provided in Section V and our conclusions are offered in Section VI. The notations used in the paper are summarized in Table I. 


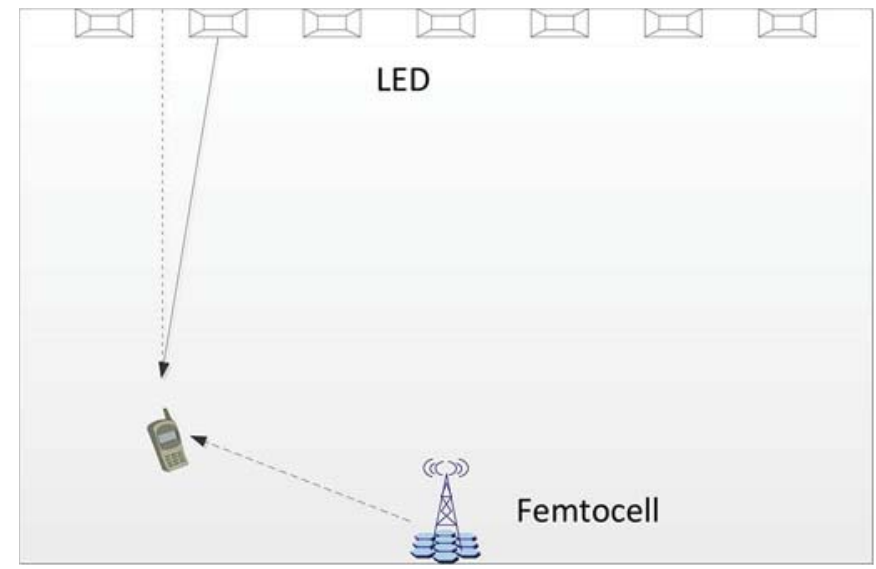

Fig. 1. The 2D model of the room using both the VLC system and the RF femtocell system.

\section{SySTEM MOdEL}

We consider a heterogeneous indoor network, where a combined VLC and a RF femtocell network are employed in a room. We denote the network set as: $\mathcal{M}=\{1,2\}$, where $m=1$ and $m=2$ represent the VLC and the RF femtocell network, respectively. It is assumed that both the VLC and the RF femtocell system are supported either by an optical fiber or powerline backbone for exchanging their roaming-related signaling information, which is unproblematic in a indoor scenario.

The set of MTs located or roaming in the indoor region is denoted by $\mathcal{N}=\{1, \cdots, n, \cdots, N\}$. We assume that the $N$ MTs are uniformly distributed in the room. Each MT for $n \in \mathcal{N}$, is capable of accessing both the VLC and the femtocell network with the same priority. Again, we consider two types of MTs, namely multi-mode MTs and multi-homing MTs. Again, the multi-mode MTs are assigned to a single network at a time, hence each multi-mode MT has to appropriately set its network selection index $x_{m, n}$, where $x_{m, n}=\{1,0\}$ indicates whether the MT $n$ is assigned to the network $m$. By contrast, for the multi-homing MTs, both the VLC and the femtocell provide resource blocks simultaneously. We denote the instantaneous transmission probability of the network $m$ transmitting to MT $n$ as $\beta_{m, n} \cdot{ }^{1}$ Furthermore, we use $\boldsymbol{x}$ and $\boldsymbol{\beta}$ to represent the network assignment matrix and RA probability matrix, respectively. The achievable throughput of MT $n$ supported by network $m$ is expressed as: $\beta_{m, n} r_{m, n}$, where $r_{m, n}$ denotes the instantaneous transmission bit rate in the DL of network $m$ to MT $n$. The VLC system has a rather different transceiver model and channel model than the traditional RF communication networks. We will now briefly introduce the achievable instantaneous transmission bit rate of both the VLC and of the RF femtocell network.

\section{A. VLC Using LED Lights}

A typical 2D-indoor VLC system using LED lights is illustrated in Fig. 1, where the LED lights are uniformly distributed on the ceiling of the room. Let us assume that $\psi_{i r}$ is the angle of

\footnotetext{
${ }^{1}$ Here $\beta_{m, n}$ may also be interpreted as the specific fraction of the normalized serving duration, during which network $m$ supports MT $n$.
}

irradiation of the LED lights, $\psi_{i n}$ is the angle of incidence, and $D_{d}$ is the distance between a transmitter and a receiver. In an optical link, the channel's direct current (DC) gain on directed path is given by [26]:

$$
H(0)= \begin{cases}\frac{(l+1) A T_{s} \kappa}{2 \pi D_{d}^{2}} \mathcal{G}, & \text { if } \psi_{\text {in }} \leq \Psi_{c}, \\ 0, & \text { if } \psi_{\text {in }}>\Psi_{c},\end{cases}
$$

where $l$ is the order of Lambertian emission, which is given by the semi-angle $\phi_{1 / 2}$ at half illumination of an LED as $l=$ $\frac{\ln 2}{\ln \left(\cos \phi_{1 / 2}\right)}$. Furthermore, $A$ is the physical area of the photodetector (PD), $T_{s}$ is the gain of the optical filter used, and $\Psi_{c}$ denotes the width of the field of view (FOV) at the receiver. Owing to the obstructions, the LOS propagation of the VLC system might be blocked. Here, the random variable $\kappa$ denotes the VLC LOS blocking event. This event is random and unpredictable. Here we assume that the event obeys the Bernoulli distribution, which indicates whether the VLC LOS reception is blocked or not. Its probability mass function may be expressed as:

$$
f(\kappa)= \begin{cases}1-p, & \text { if } \kappa=1 \\ p, & \text { if } \kappa=0\end{cases}
$$

where $p$ denotes the VLC LOS blocking probability. Equation (1) indicates that once the angle of irradiation of a receiver is higher than the Field of View (FOV), the receiver's LOS would be blocked. We assume that $\mathcal{G}=\cos ^{l}\left(\psi_{i r}\right) g\left(\psi_{\text {in }}\right) \cos \left(\psi_{\text {in }}\right)$, where $g\left(\psi_{i n}\right)$ is the gain of an optical concentrator [27] and can be characterized as [28]:

$$
g\left(\psi_{i n}\right)= \begin{cases}\frac{l^{2}}{\sin ^{2} \Psi_{c}}, & \text { if } \psi_{i n} \leq \Psi_{c} \\ 0, & \text { if } \psi_{i n}>\Psi_{c} .\end{cases}
$$

Apart from the VLC LOS reception, the MTs may also receive the VLC reflected path owing to the wall. The received optical power is given by the channel's DC gain on the direct LOS path $H_{d}(0)$ and reflected path $H_{r e f}(0)$, which may be written as:

$$
P_{r}=\sum^{\text {LEDs }}\left[P_{t} H_{d}(0)+\int_{\text {walls }} P_{t} d H_{r e f}(0)\right],
$$

where $P_{t}$ and $P_{r}$ denotes the transmitted and received optical power, respectively. The channel DC gain on the first reflection $d H_{\text {ref }}$ is given by [27]:

$$
d H_{\text {ref }}(0)= \begin{cases}\frac{(l+1) A T_{s} \rho}{2 \pi^{2} D_{1}^{2} D_{2}^{2}} \mathcal{J} \mathcal{G} d A_{\text {wall }}, & \text { if } \psi_{\text {in }} \leq \Psi_{c}, \\ 0, & \text { if } \psi_{\text {in }}>\Psi_{c}\end{cases}
$$

where $D_{1}$ is the distance between an LED light and a reflecting surface, $D_{2}$ denotes the distance between a reflective point and a MT receiver, $\rho$ is the reflectance factor, whilst $d A_{\text {wall }}$ is a small reflective area. Furthermore, we assume that $\mathcal{J}=\cos \left(\omega_{1}\right) \cos \left(\omega_{2}\right)$, where $\omega_{1}$ and $\omega_{2}$ denote the angle of irradiance to a reflective point and the angle of irradiance to a MT's receiver, respectively.

The dominant noise contribution is assumed to be the shot noise due to ambient light from windows. We also take thermal noise into account. Hence, the receiver filter's output contains Gaussian noise having a total variance of $\sigma_{\mathrm{VLC}}^{2}$ given by the 
sum of contributions from both the shot noise and the thermal noise, which is expressed as:

$$
\sigma_{\mathrm{VLC}}^{2}=\sigma_{\text {shot }}^{2}+\sigma_{\text {thermal }}^{2}
$$

According to [27], the variance of shot noise $\sigma_{\text {shot }}^{2}$ is given by:

$$
\sigma_{\text {shot }}^{2}=2 q \varsigma P_{r} B+2 q I_{b g} I_{1} B,
$$

where $q$ is the electronic charge, $\varsigma$ is the detector's responsivity. $B$ is the equivalent noise bandwidth and $I_{b g}$ is the background current caused by the background light. Furthermore, the variance of thermal noise is given by [27]:

$$
\sigma_{\text {thermal }}^{2}=\frac{8 \pi \varrho T_{K}}{G} \eta A I_{1} B^{2}+\frac{16 \pi^{2} \varrho T_{k} \Gamma}{g_{m}} \eta^{2} A^{2} I_{2} B^{3},
$$

where $\varrho$ is the Boltzmann's constant, $T_{K}$ is the absolute temperature, $G$ is the open-loop voltage gain, $\eta$ is the fixed capacitance of the PD per unit area, $\Gamma$ is the FET channel noise factor, and finally, $g_{m}$ is the FET transconductance. The noise bandwidth factors $I_{1}$ and $I_{2}$ are constant experimental values. Here, we opt for the typical values used in the literature [27], [28], namely for $I_{1}=0.562$ and $I_{2}=0.0868$.

Let us define the receiver's SNR in the VLC system's MT $n$ as $\gamma_{1, n}$, which is given by:

$$
\gamma_{1, n}=\frac{P_{r}}{\sigma_{\mathrm{VLC}}^{2}} .
$$

We assume that the VLC system relies on M-PAM based transmission. According to [7], the relationship between the bit error ratio (BER) and SNR for M-PAM signals is approximated as:

$$
\mathrm{BER}_{\mathrm{M}-\mathrm{PAM}} \approx \frac{Z-1}{Z} \frac{2}{\log _{2} Z} Q\left(\frac{\sqrt{\gamma_{1, n} / 2}}{Z-1}\right),
$$

which $Z$ is the number of constellation points. As a result, the maximum affordable $Z$ value may be found for a given BER target. Then, the achievable transmission rate of the VLC system is given by:

$$
R=B \log _{2} Z
$$

\section{B. Channel Model of the RF Femtocell}

In this paper, we assume that the DL channel of the femtocell to a MT is subject to uncorrelated Rayleigh fading ${ }^{2}$ obeying a unity average power constraint and inverse- $\iota$ th power pathloss. Hence the instantaneous transmission bit rate of the RF femtocell is given by the classical Shannon capacity of:

$$
R=B_{f} \log _{2}\left(1+\frac{P_{f}}{\sigma^{2}} \Pi A_{f} d^{-\iota} h\right),
$$

where $B_{f}$ denotes the bandwidth of the RF femtocell, $P_{f}$ denotes the DL transmission power of the femtocell's radio port. Let us now employ continuous rate adaptation and let $\Pi$

\footnotetext{
${ }^{2}$ Since we may not be able to guarantee line of sight transmission for the $\mathrm{RF}$ femtocell system, here we consider the worst-case scenario, where the DL channel of the RF femtocell system is modeled as the widely used Rayleigh channel.
}

be the received SNR discrepancy from the continuous input memoryless channel's capacity at the target BER, which is given by: $\Pi=-\frac{1.5}{\log (5 \mathrm{BER})}$ [29]. Furthermore, $A_{f}$ is a constant that depends on the carrier frequency $f_{c}, d$ denotes the distance of this link, while $\iota$ is the pathloss exponent and finally, $h$ is the Rayleigh-distributed channel gain. Let us define the receiver's $S N R$ in the DL of the femtocell to MT $n$ as $\gamma_{2, n}$, which is given by:

$$
\gamma_{2, n}=\frac{P_{f}}{\sigma^{2}} A_{f} d^{-\iota} h,
$$

where $\sigma^{2}$ denotes the variance of the AWGN. The probability density of function (PDF) of the SNR $\gamma_{2, n}$ of a Rayleighchannel obeys the exponential-distribution of:

$$
f_{\gamma_{2, n}}(x)=\frac{1}{\Gamma} e^{-\frac{1}{\Gamma} x},
$$

where we have $\Gamma=\frac{P_{f} \Pi A_{f} d^{-\iota}}{\sigma^{2}}$.

\section{Effective Capacity}

In next-generation networks, it is essential to consider a range of QoS metrics, such as the achievable data rate, the tolerable delay and the delay-violation probability. During the early 90's, statistical QoS guarantees have been extensively studied in the context of effective bandwidth theory of [30]-[32]. The theory states that for a dynamic queuing system having stationary ergodic arrival and service processes, the probability of exceeding a certain queue length decays exponentially. Inspired by the effective bandwidth theory, the authors of [13] proposed a link-layer metric termed as the effective capacity, which characterizes the effect of delay on the system. Owing to its advantages, the effective capacity has been widely adopted [13]-[17] for studying the steady-state delay-target violation probability. According to [13], for a dynamic queuing system where the arrival and service processes are stationary and ergodic, the probability that the queue length $D(t)$ at an instant $t$ of a service exceeds the maximum tolerable delay bound $D_{\max }$ is given by:

$$
\operatorname{Pr}\left\{D(t) \geq D_{\max }\right\} \approx \gamma(t) e^{-\theta D_{\max }},
$$

where $\gamma(t)=\operatorname{Pr}\{D(t) \geq 0\}$ is the probability that the transmission queuing buffer is non-empty at a randomly selected instant $t$. Note from (15) that $\theta \geq 0$ is a crucial parameter, directly characterizing the exponential decaying rate of the probability that the delay exceeds $D_{\max }$. As a result, $\theta$ may be referred to as delay-related QoS exponent of a connection.

The effective capacity may also be interpreted as the maximum constant packet-arrival rate that the system is capable of supporting, without violating a given delay-related QoS requirement indicated by the $\mathrm{QoS}$ exponent $\theta$. For uncorrelated block fading channels wherein the service process is uncorrelated, the effective capacity is defined as [13], [33]:

$$
\Delta(\theta)=-\frac{1}{\theta} \ln \mathbb{E}[\exp (-\theta r)],
$$

where $\mathbb{E}(\cdot)$ is the expectation operator and $r$ denotes the throughput. 
1) Effective Capacity of VLC: Given the position of a MT $n$, if the angle of irradiation of a receiver is higher than the FOV, the effective capacity of VLC $\Delta_{1, n}$ is equal to 0 . However, if the angle of irradiation of a receiver is lower than the FOV, the instantaneous transmission rate $r_{1, n}$ obeys a Bernoulli distribution, with the probability mass function of:

$$
f\left(r_{1, n}\right)= \begin{cases}1-p, & \text { if } r_{1, n}=\mathcal{R}_{1, n} \\ p, & \text { if } r_{1, n}=\mathcal{R}_{2, n},\end{cases}
$$

where $\mathcal{R}_{1, n}$ and $\mathcal{R}_{2, n}$ denote the achievable transmission rate from VLC system to MT $n$ with and without VLC LOS reception, respectively. As a result, the VLC transmission channel is modeled as a two-rates channel.

Based on the above discussions, the VLC system's effective capacity, which is again the maximum constant packet-arrival rate that the service is capable of supporting under the statistical delay limit of the MT $n$ specified by $\theta_{n}$, may be expressed as:

$$
\Delta_{1, n}= \begin{cases}-\frac{1}{\theta_{n}} \ln \mathcal{Q}, & \text { if } \psi_{i n} \leq \Psi_{c}, \\ 0, & \text { if } \psi_{i n}>\Psi_{c}\end{cases}
$$

where we have $\mathcal{Q}=p e^{-\theta_{n} \beta_{1, n} \mathcal{R}_{2, n}}+(1-p) e^{-\theta_{n} \beta_{1, n} \mathcal{R}_{1, n}}$

2) Effective Capacity of the RF Femtocell: According to (12), (14), and (16), the effective capacity of the RF femtocell system to MT $n$ is given by:

$$
\begin{aligned}
\Delta_{2, n} & =-\frac{1}{\theta_{n}} \ln \int_{0}^{\infty} e^{-\theta_{n} \beta_{2, n} B_{f} \log _{2}(1+x)} f_{\gamma_{2, n}}(x) d x \\
& =-\frac{1}{\theta_{n}} \ln \left[\frac{1}{\Gamma} \int_{0}^{\infty}(1+x)^{-\frac{\theta_{n} \beta_{2, n} B_{f}}{\ln 2}} e^{-\frac{x}{\Gamma}} d x\right] \\
& =-\frac{1}{\theta_{n}} \ln \left(\Gamma^{-\frac{\theta_{n} \beta_{2, n} B_{f}}{2 \ln 2}} e^{\left.\frac{1}{2 \Gamma} \mathcal{T}\right)}\right. \\
& =\frac{\beta_{2, n} B_{f}}{2 \ln 2} \ln \Gamma-\frac{1}{2 \theta_{n} \Gamma}-\frac{1}{\theta_{n}} \ln \mathcal{T},
\end{aligned}
$$

where $\mathcal{T}=\mathcal{W}_{-} \frac{\theta_{n} \beta_{2, n} B_{f}}{2 \ln 2}, \frac{\ln 2-\theta_{n} \beta_{2, n} B_{f}}{2 \ln 2}\left(\frac{1}{\Gamma}\right)$, and $\mathcal{W}$.,. (.) represents the Whittaker functions [34].

Note from the (18) and (19), the effective capacity of the network $m$ to MT $n$ is the function of $\beta_{m, n}$. In the following sections, we will use $\Delta_{m, n}$ to represent $\Delta_{m, n}\left(\beta_{m, n}\right)$.

\section{PROBLEM FORMULATION}

In this section, the RA problem formulation is presented for both multi-homing MTs and multi-mode MTs in the indoor HetNet considered. Let us now adopt a utility function based perspective, assuming that the MT $n$ obtains utility $U_{m, n}\left(\beta_{m, n}\right)$ from network $m$.

\section{A. Utility Function: $\alpha$-Proportional Fairness Approach}

In this paper, we consider the RA problem under the consideration of the concept fairness. The mathematical concept of fairness is formulated as an optimization problems. The fairness optimization problem may be interpreted as a throughput maximization problem [35], as a max-min fairness problem [35] and as a proportional fairness problem [36]. Here a generic fairness notion referred to $\alpha$-proportional fairness [20]-[23] is introduced, which embodies a number of fairness concepts, including the above-mentioned three problems by appropriately adjusting the values of the parameter $\alpha$. We define the utility $U_{m, n}\left(\beta_{m, n}\right)$ as:

$$
U_{m, n}\left(\beta_{m, n}\right)=\varphi_{\alpha}\left(\Delta_{m, n}\right),
$$

where $\varphi_{\alpha}(\cdot)$ denotes the $\alpha$-proportional fairness defined in [21], where $\varphi_{\alpha}(\cdot)$ is a monotonically increasing, strictly concave and continuously differentiable function, which may be expressed as:

$$
\varphi_{\alpha}(x)= \begin{cases}\log (x), & \text { if } \alpha=1 \\ \frac{x^{1-\alpha}}{1-\alpha}, & \text { if } \alpha \geq 0, \alpha \neq 1 .\end{cases}
$$

We note that for different values of $\alpha$, maximizing our utility function $U_{m, n}\left(\beta_{m, n}\right)$ reduces to several well-known fairness concepts. For example, the maximum effective capacity is achieved for $\alpha=0$ [35], proportional fairness is achieved for $\alpha=1$ [36] and max-min fairness [35] is obtained, when we have $\alpha \rightarrow \infty$.

\section{B. Problem Formulation for Multi-Homing MTs}

When the MTs are capable of multi-homing, both the VLC and the RF femtocell networks may allocate resource blocks for simultaneously supporting the MTs. We set out to maximize the overall effective capacity of all indoor MTs. As a result, the corresponding RA problem may be formulated as:

$$
\begin{aligned}
\text { Problem 1: } & \underset{\boldsymbol{\beta}}{\operatorname{maximize}} \sum_{n \in \mathcal{N}} \varphi_{\alpha}\left(\sum_{m \in \mathcal{M}} \Delta_{m, n}\right) \\
\text { subject to : } & \sum_{m \in \mathcal{M}} \Delta_{m, n} \geq R_{n}, \forall n \in \mathcal{N}, \\
& \sum_{n \in \mathcal{N}} \beta_{m, n} \leq 1, \forall m \in \mathcal{M}, \\
& 0 \leq \beta_{m, n} \leq 1 .
\end{aligned}
$$

The effective capacity $\Delta_{1, n}$ of the VLC and $\Delta_{2, n}$ of the femtocell is given by (18) and (19), respectively. Physically, the constraint (23) ensures that the HetNet is capable of satisfying the bit-rate $R_{n}$ of the MT $n$, while the constraint (24) guarantees that the total transmission probability for each of the networks should always be less than 1. Finally, the constraint (25) describes the feasible region of the optimization variables.

Lemma 1: The RA problem described by (22)-(25) is a concave optimization problem.

Proof: See Appendix A.

While the Problem 1 of (22)-(25) may be solved in a centralized manner with the aid of a central resource manager, this is not a viable practical solution, when the available networks are operated by different service providers.

\section{Problem Formulation for Multi-Mode MTs}

When multi-mode MTs are considered, only a single network supports the MT at a time. Then, the RA problem may be formulated as that of maximizing the total utility $U_{m, n}\left(\beta_{m, n}\right)$ 
under the constraint of the QoS requirements expressed in terms of each MT's overall effective capacity, fairness and delay as follows:

$$
\begin{aligned}
\text { Problem 2: } & \max _{\boldsymbol{x}, \boldsymbol{\beta}} \sum_{n \in \mathcal{N}} \varphi_{\alpha}\left(\sum_{m \in \mathcal{M}} x_{m, n} \Delta_{m, n}\right) \\
\text { subject to : } & \sum_{m \in \mathcal{M}} x_{m, n} \Delta_{m, n} \geq R_{n}, \forall n \in \mathcal{N}, \\
& \sum_{n \in \mathcal{N}} x_{m, n} \beta_{m, n} \leq 1, \forall m \in \mathcal{M}, \\
& \sum_{m \in \mathcal{M}} x_{m, n}=1, \\
& x_{m, n}=\{1,0\}, \quad 0 \leq \beta_{m, n} \leq 1 .
\end{aligned}
$$

The effective capacity of VLC $\Delta_{1, n}$ and femtocell $\Delta_{2, n}$ is given by (18) and (19), respectively.

Under this formulation, the variables to be optimized are $x_{m, n}$ and $\beta_{m, n}, \forall m, n$. Physically, the constraint (27) ensures that the HetNet is capable of satisfying the bit-rate $R_{n}$ of the MT $n$, while the constraint (28) guarantees that the total transmission probability for each of the networks should always be less than 1. Furthermore, the constraint (29) guarantees that each MT should always select only one of the networks for its transmissions. Finally, the constraint (30) describes the feasible region of the optimization variables.

We note that Problem 2 is a mixed integer non-linear programming (MINLP) problem that involves both binary variables $x_{m, n}$ and real-valued positive variables $\beta_{m, n}$ during optimization. In general, MINLP problems are mathematically intractable. Nonetheless, recently several optimization tools have been developed for solving MINLP problems. The BONMIN solver [37] is for example capable of solving smooth, twice differentiable, mixed integer nonlinear programs, which was deployed for providing the upper-bound benchmark solution.

\section{Decentralized Sub-Optimal Resource ALLOCATION SCHEMES}

Problem 1 and Problem 2 may be solved with the aid of centralized optimization tools. However, in order to reduce the computational complexity and to make the problem tractable, in this section we propose decentralized sub-optimal RA schemes.

\section{A. Decentralized Solution for Multi-Homing MTs}

Since the problem is a concave one, convex duality implies that the optimal solution to this problem may be found from the Lagrangian formulation [24]. The Lagrangian function for (22) under the constraints of (23)-(25) can be expressed as:

$$
\begin{aligned}
L(\boldsymbol{\beta}, \boldsymbol{\lambda}, \boldsymbol{\mu})=\sum_{n \in \mathcal{N}}[ & \varphi_{\alpha}\left(\sum_{m \in \mathcal{M}} \Delta_{m, n}\right)-\sum_{m \in \mathcal{M}} \lambda_{m} \beta_{m, n} \\
& \left.+\sum_{m \in \mathcal{M}} \mu_{n} \Delta_{m, n}\right]+\sum_{m \in \mathcal{M}} \lambda_{m}-\sum_{n \in \mathcal{N}} \mu_{n} R_{n},
\end{aligned}
$$

where we have $\mathbf{0} \leq \boldsymbol{\beta} \leq \mathbf{1}$, while $\mu_{n}$ and $\lambda_{m}$ are the Lagrange multipliers or prices associated with the $n$th inequality constraint (23) and with the $n$th inequality constraint (24), respectively. The dual objective function $g(\boldsymbol{\lambda}, \boldsymbol{\mu})$ is defined as the maximum value of the Lagrangian over $\boldsymbol{\beta}$, which is expressed as:

$$
g(\boldsymbol{\lambda}, \boldsymbol{\mu})=\sup _{\boldsymbol{\beta}} L(\boldsymbol{\beta}, \boldsymbol{\lambda}, \boldsymbol{\mu}) .
$$

The dual variables $(\boldsymbol{\lambda}, \boldsymbol{\mu})$ are dual feasible if we have $\boldsymbol{\lambda} \geq$ $0, \boldsymbol{\mu} \geq 0$. The dual function can then be maximized for finding an upper bound on the optimal value of the original problem (22):

$$
\begin{array}{cc} 
& \min _{\boldsymbol{\lambda}, \boldsymbol{\mu}} g(\boldsymbol{\lambda}, \boldsymbol{\mu}) \\
\text { sub } \quad \boldsymbol{\lambda} \geq 0, \boldsymbol{\mu} \geq 0,
\end{array}
$$

which is always a convex optimization problem. The difference between the optimal primal objective and the optimal dual objective is referred to as the duality gap, which is always nonnegative. A central result in convex analysis showed that when the problem is convex, the duality gap reduces to zero at the optimum [24], [38]. Hence, the primal problem of (22) can be equivalently solved by solving the dual problem of (33). In principle, the dual problem may be readily solved using standard routines, such as the Newton method and the barrier method [24]. However, these algorithms generally involve centralized computation and require global knowledge of all parameters. Hence, we propose an optimal decentralized RA algorithm for solving the problem using full dual decomposition [25].

Recall that in (32) we defined a dual objective function $g_{n}\left(\lambda_{m}, \mu_{n}\right)$ for MT $n$, which may be written as:

$$
\begin{aligned}
g_{n}\left(\lambda_{m}, \mu_{n}\right)= & \varphi_{\alpha}\left(\sum_{m \in \mathcal{M}} \Delta_{m, n}\right)-\sum_{m \in \mathcal{M}} \lambda_{m} \beta_{m, n} \\
& +\sum_{m \in \mathcal{M}} \mu_{n} \Delta_{m, n} .
\end{aligned}
$$

Our primal problem described by (22)-(25) may be separated into two levels of optimization. At the lower level, we decouple the problem of (32) into $N$ subproblems, where the $n t h$ subproblem may be written as:

$$
\beta_{m, n}^{\star}=\arg \max _{0 \leq \beta_{m, n} \leq 1} g_{n}\left(\lambda_{m}, \mu_{n}\right), \forall m \in \mathcal{M} .
$$

It may be shown that $g_{n}\left(\lambda_{m}, \mu_{n}\right)$ is concave with respect to the variable $\beta_{m, n}$. Hence the maximization of $g_{n}\left(\lambda_{m}, \mu_{n}\right)$ may be achieved by finding the partial derivative of $g_{n}\left(\lambda_{m}, \mu_{n}\right)$ with respect to $\beta_{m, n}$, which is given by:

$$
\frac{\partial g_{n}\left(\lambda_{m}, \mu_{n}\right)}{\partial \beta_{m, n}}=\frac{\Delta_{m, n}^{\prime}}{\sum_{m \in \mathcal{M}} \Delta_{m, n}}+\mu_{n} \Delta_{m, n}^{\prime}-\lambda_{m},
$$


where $\Delta_{m, n}^{\prime}=\frac{d \Delta_{m, n}}{d \beta_{m, n}}$. Furthermore, the second partial derivative of $g_{n}\left(\lambda_{m}, \mu_{n}\right)$ is given by:

$$
\begin{aligned}
\frac{\partial^{2} g_{n}\left(\lambda_{m}, \mu_{n}\right)}{\partial \beta_{m, n}^{2}}= & \mu_{n} \Delta_{m, n}^{\prime \prime}+\frac{\Delta_{m, n}^{\prime \prime} \sum_{m \in \mathcal{M}} \Delta_{m, n}}{\left(\sum_{m \in \mathcal{M}} \Delta_{m, n}\right)^{2}} \\
& -\frac{\left(\Delta_{m, n}^{\prime}\right)^{2}}{\left(\sum_{m \in \mathcal{M}} \Delta_{m, n}\right)^{2}} \leq 0 \\
\frac{\partial^{2} g_{n}\left(\lambda_{m}, \mu_{n}\right)}{\partial \beta_{i, n} \partial \beta_{j, n}}= & -\frac{\Delta_{i, n}^{\prime} \Delta_{i, n}^{\prime}}{\left(\sum_{m \in \mathcal{M}} \Delta_{m, n}\right)^{2}} \leq 0 .
\end{aligned}
$$

As a result, $\frac{\partial g_{n}\left(\lambda_{m}, \mu_{n}\right)}{\partial \beta_{m}, n}$ is a monotonically decreasing function with respect to $\beta_{m, n}$ for all $m$. If we have $\left[\frac{\partial g_{n}\left(\lambda_{m}, \mu_{n}\right)}{\partial \beta_{m, n}} \mid \beta_{m, n}=\right.$ $0] \leq 0$, then we may have $\beta_{m, n}^{\star}=0, \forall m$. If we have $\left[\frac{\partial g_{n}\left(\lambda_{m}, \mu_{n}\right)}{\partial \beta_{m}, n} \mid \beta_{m, n}=1\right] \geq 0$, then we may have $\beta_{m, n}^{\star}=1, \forall m$. Otherwise, $\beta_{m, n}^{\star}$ may be derived by solving the following Equation for each network $m$ :

$$
\frac{\Delta_{m, n}^{\prime}}{\sum_{m \in \mathcal{M}} \Delta_{m, n}}+\mu_{n} \Delta_{m, n}^{\prime}-\lambda_{m}=0
$$

which may be solved by the steepest descent method [24]. ${ }^{3}$

At the higher level, we have the master dual problem, which may be expressed as:

$$
\min _{\boldsymbol{\lambda}, \boldsymbol{\mu}} g(\boldsymbol{\lambda}, \boldsymbol{\mu}),
$$

where we have $g(\boldsymbol{\lambda}, \boldsymbol{\mu})=\sum_{m \in \mathcal{M}} \sum_{n \in \mathcal{N}} g_{m, n}\left(\beta_{m, n}^{\star}, \lambda_{m}, \mu_{n}+\right.$ $\sum_{m \in \mathcal{M}} \lambda_{m}-\sum_{n \in \mathcal{N}} \mu_{n} R_{n}$, and $\beta_{m, n}^{\star}$ denotes the optimal value derived from the lower level optimization problem of (35). Since the function $g(\boldsymbol{\lambda}, \boldsymbol{\mu})$ is concave and differentiable, we can use a gradient method for solving the master dual problem, as a benefit of its simplicity. Instead of minimizing the function directly with respect to $\boldsymbol{\lambda}$ and $\boldsymbol{\mu}$, it can be minimized over a single set of Lagrange multipliers first, and then over the remaining one, which may be formulated as minimum $\left.\operatorname{minimum}_{\boldsymbol{\lambda} \geq 0} g(\boldsymbol{\lambda}, \boldsymbol{\mu})\right]$.

Firstly, we solve the minimization problem for a given $\boldsymbol{\mu}$. Then the derivative of $g(\boldsymbol{\lambda}, \boldsymbol{\mu})$ with respect to $\boldsymbol{\lambda}$ is written as:

$$
\frac{\partial g(\boldsymbol{\lambda}, \boldsymbol{\mu})}{\partial \boldsymbol{\lambda}}=1-\sum_{n \in \mathcal{N}} \beta_{m, n}^{\star}
$$

As a result, the price parameter $\boldsymbol{\lambda}$ is updated according to:

$$
\lambda_{m}(t+1)=\left[\lambda_{m}(t)-\xi_{\lambda}(t)\left(1-\sum_{n \in \mathcal{N}} \beta_{m, n}^{\star}\right)\right]^{+},
$$

\footnotetext{
${ }^{3}$ Nonlinear equations can be solved by several numerical methods. However, due to the length limitation of our paper, we will not be able to present the steepest descent method here.
}

TABLE II

Decentralized Algorithm for Problem 1

\begin{tabular}{l}
\hline Algorithm $\mathbf{1}$ \\
\hline Input \\
$\theta_{n}$ : Delay requirement of each MT $n \forall n$, \\
$R_{n}$ : Rate requirement of each MT $n \forall n$ \\
Initialization \\
$t \leftarrow 1$; \\
Price value for each network: $\boldsymbol{\lambda}(t)=\left\{\lambda_{1}(t), \ldots, \lambda_{M}(t)\right\}=\mathbf{0}$ \\
Price value for each MT: $\boldsymbol{\mu}(t)=\left\{\mu_{1}(t), \ldots, \mu_{N}(t)\right\}=\mathbf{0}$ \\
step size: positive $\xi$ and epsilon \\
While $t$ does not reach its maximum \\
$\quad$ Get optimal $\boldsymbol{\beta}$ \\
$\quad$ For each MT $n \in \mathcal{N}$ \\
$\quad$ Each MT solves the problem presented in Eq. (35); \\
$\quad$ End for \\
Update price values \\
$\quad$ For $m \in \mathcal{M}$ \\
$\quad$ Price value $\lambda_{m}$ updates according to Eq. (42) \\
End for \\
$\quad$ For $n \in \mathcal{N}$ \\
$\quad$ Price value $\mu_{n}$ updates according to Eq. (43) \\
End for \\
$\quad \xi \leftarrow \xi t^{-\frac{1}{2}+\epsilon}$ \\
$\quad t \leftarrow t+1$ \\
End \\
\hline Output $\boldsymbol{\beta}^{\star}(t)$ \\
\hline
\end{tabular}

where $[.]^{+}$is a projection on the positive orthant to account for the fact that we have $\lambda_{m} \geq 0$. Furthermore, $\xi_{\lambda}(t)$ denotes the step-size taken in the direction of the negative gradient for the price parameter $\lambda$ at iteration $t$. In order to guarantee convergence, where we have to satisfy $\lim _{t \rightarrow \infty} \xi_{\lambda}(t)=0$ and $\sum_{t=0}^{\infty} \xi_{\lambda}(t)=\infty$. In this paper, we set $\xi_{\lambda}(t)=\xi t^{-\frac{1}{2}+\epsilon}, \xi$ and $\epsilon$ are positive constants.

Then, the price parameter $\boldsymbol{\mu}$ is similarly updated according to:

$$
\mu_{n}(t+1)=\left[\mu_{n}(t)-\xi_{\mu}(t)\left(\sum_{m \in \mathcal{M}} \Delta_{m, n}-R_{n}\right)\right]^{+} .
$$

Based on the above discussions, our decentralized optimal RA scheme is constituted by an iterative algorithm, which determines the optimal transmission probability in the DL of network $m$ to MT $n$ based on the update of a pair of price parameters $\lambda_{m}$ and $\mu_{n}$, over a number of iterations, until the optimal solution is found. Each of the networks $m$ is initialized to a feasible price value $\lambda_{m}$, while each MT $n$ is initialized to a feasible price value $\mu_{n}$. Each MT broadcasts its price value to all the available networks. Then each MT calculates the optimal transmission probability based on the price information $(\boldsymbol{\lambda}, \boldsymbol{\mu})$ and the optimal transmission probability is derived during the last iteration. Each of the networks $m$ updates its price value $\lambda_{m}$ based on the newly derived optimal transmission probability $\beta$ and then broadcasts the optimal transmission probability to the MTs. Similarly, each MT updates its price value $\mu_{n}$ based on the optimal transmission probability. The MTs broadcast their new price values $\boldsymbol{\mu}$ to the networks and the process continues, until the algorithm converges. The decentralized optimal RA algorithm is formally described in Table II. 


\section{B. Decentralized Solution for Multi-Mode MTs}

Problem 2 of (26)-(30) is formulated as a MINLP and may be computationally intractable. A potentially straightforward solution may be to derive firstly the optimal resource block $\boldsymbol{\beta}$ for a specific $\boldsymbol{x}$, then to exhaustive search through the entire set of all the possible $\boldsymbol{x}$ values. For a system having access to $M$ networks and $N$ MTs, there are $M^{N}$ combinations for the network selection indicator variables $\boldsymbol{x}$. Therefore, a simpler solution may be found by relaxing the binary constraint imposed on the network selection indicator variables $x_{m, n}$, so that they may assume continuous values from the interval $[0,1]$. Naturally, the original problem is not actually solved by the relaxation of the binary constraint. However, it has been shown in [39] that solving the dual of the relaxed problem provides solutions that are arbitrarily close to the original, non-relaxed problem.

Since $x_{m, n}$ assumes either the values of 0 or 1 , there is exactly one $x_{m, n}=1$ value for each MT $n$. If we denote such a specific network by the index $m^{\prime}$, we have $\varphi_{\alpha}\left(\sum_{m \in \mathcal{M}}\right.$ $\left.x_{m, n} \Delta_{m, n}\right)=\varphi_{\alpha}\left(\Delta_{m^{\prime}, n}\right)$. Thus, the objective function in (26) is equivalent to the following function:

$$
\max _{\boldsymbol{x}, \boldsymbol{\beta}} \sum_{n \in \mathcal{N}} \sum_{m \in \mathcal{M}} x_{m, n} \varphi_{\alpha}\left(\Delta_{m, n}\right) .
$$

The equivalent relaxed optimization problem of (26)-(30) is reformulated as follows:

$$
\begin{aligned}
\text { Problem 3: } & \max _{\boldsymbol{x}, \boldsymbol{\beta}} \sum_{m \in \mathcal{M}} \sum_{n \in \mathcal{N}} x_{m, n} \varphi_{\alpha}(\Delta m, n) \\
\text { subject to : } & \sum_{m \in \mathcal{M}} x_{m, n} \Delta_{m, n} \geq R_{n}, \forall n \in \mathcal{N}, \\
& \sum_{n \in \mathcal{N}} x_{m, n} \beta_{m, n} \leq 1, \forall m \in \mathcal{M}, \\
& \sum_{m \in \mathcal{M}} x_{m, n}=1, \\
& 0 \leq x_{m, n} \leq 1, \quad 0 \leq \beta_{m, n} \leq 1 .
\end{aligned}
$$

In this formulation, the variables to be optimized are $x_{m, n}$ and $\beta_{m, n}, \forall m, n$.

Lemma 2: The RA problem described by (45)-(49) is a concave optimization problem with respect to the variables $x_{m, n}$ and $\beta_{m, n}$.

Proof: See Appendix B.

Since Problem 3 is also based on a concave function, the optimal solution may be found from the Lagrangian formulation, which may be written as:

$$
\begin{aligned}
& L(\boldsymbol{x}, \boldsymbol{\beta}, \boldsymbol{\lambda}, \boldsymbol{\mu})=\sum_{m \in \mathcal{M}} \sum_{n \in \mathcal{N}}\left[x_{m, n} \varphi_{\alpha}\left(\Delta_{m, n}\right)\right. \\
& \left.-\lambda_{m} \beta_{m, n}+\mu_{n} x_{m, n} \Delta_{m, n}\right]+\sum_{m \in \mathcal{M}} \lambda_{m}-\sum_{n \in \mathcal{N}} \mu_{n} R_{n},
\end{aligned}
$$

where we have $\mathbf{0} \leq \boldsymbol{x} \leq \mathbf{1}, \mathbf{0} \leq \boldsymbol{\beta} \leq \mathbf{1}$ and $\sum_{m \in \mathcal{M}} x_{m, n}=1$. Furthermore, $\mu_{n}$ and $\lambda_{m}$ are the Lagrange multipliers or prices associated with the $n$th inequality constraint of (46) and with the $m$ th inequality constraint of (47), respectively. The optimal RA variables $\boldsymbol{x}, \boldsymbol{\beta}$ may be obtained by solving:

$$
\min _{\boldsymbol{\lambda}, \boldsymbol{\mu}} \max _{\boldsymbol{x}, \boldsymbol{\beta}} L(\boldsymbol{x}, \boldsymbol{\beta}, \boldsymbol{\lambda}, \boldsymbol{\mu}) .
$$

Similarly to Section IV-A, a decentralized optimal RA algorithm can be proposed for solving the problem using full dual decomposition.

We define a dual objective function $g_{n}\left(x_{m, n}, \beta_{m, n}\right)$ for MT $n$, which may be written as:

$$
\begin{aligned}
g_{n}\left(x_{m, n}, \beta_{m, n}\right) & =\sum_{m \in \mathcal{M}} x_{m, n} \varphi_{\alpha}\left(\Delta_{m, n}\right) \\
& -\sum_{m \in \mathcal{M}} \lambda_{m} \beta_{m, n}+\sum_{m \in \mathcal{M}} \mu_{n} x_{m, n} \Delta_{m, n} .
\end{aligned}
$$

At the lower level, we have the subproblems, one for each $n$, which may be written as:

$$
\left\{x_{m, n}^{\star}, \beta_{m, n}^{\star}\right\}=\arg \max _{0 \leq x_{m, n} \leq 1,0 \leq \beta_{m, n} \leq 1} g_{n}\left(x_{m, n}, \beta_{m, n}\right) .
$$

Similarly, $g_{n}\left(x_{m, n}, \beta_{m, n}\right)$ is concave with respect to the variable $x_{m, n}$ and $\beta_{m, n}$. Hence the maximization of $g_{n}\left(x_{m, n}, \beta_{m, n}\right)$ may be derived by finding the partial derivative of $g_{n}\left(x_{m, n}\right.$, $\left.\beta_{m, n}\right)$, which is given by:

$$
\begin{aligned}
\frac{\partial g_{n}}{\partial x_{m, n}} & =\varphi_{\alpha}\left(\Delta_{m, n}\right)+\mu_{n} \Delta_{m, n}-\lambda_{m} \beta_{m, n} \\
\frac{\partial g_{n}}{\partial \beta_{m, n}} & =x_{m, n}\left(\frac{\Delta^{\prime}}{\Delta_{m, n}}+\mu_{n} \Delta_{m, n}^{\prime}-\lambda_{m}\right) .
\end{aligned}
$$

We will firstly derive the optimal RA probability $\beta_{m, n}^{\star}$ according to (55). Since the second partial derivative of $g_{n}\left(x_{m, n}\right.$, $\left.\beta_{m, n}\right)$ with respect to $\beta_{m, n}$ is non-positive, $\beta_{m, n}^{\star}$ is derived according to the following criterion:

1) If $\frac{\Delta_{m, n}^{\prime}(1)}{\Delta_{m, m}(1)}+\mu_{n} \Delta_{m, n}^{\prime}(1)-\lambda_{m} \geq 0$, then $\beta_{m, n}^{\star}=1$;

2) If $\frac{\Delta_{m, n}^{\prime}(0)}{\Delta_{m, m}(0)}+\mu_{n} \Delta_{m, n}^{\prime}(0)-\lambda_{m} \leq 0$, then $\beta_{m, n}^{\star}=0$;

3) Else, the optimal RA probability $\beta_{m, n}^{\star}$ from network $m$ and MT $n$ is derived by solving the equation $\frac{\Delta_{m, n}^{\prime}\left(\beta_{m, n}^{\star}\right)}{\Delta_{m, m}\left(\beta_{m, n}^{\star}\right)}+\mu_{n} \Delta_{m, n}^{\prime}\left(\beta_{m, n}^{\star}\right)-\lambda_{m}=0$.

The optimal network selection index $x_{m, n}^{\star}$ is then determined according to the $\beta_{m, n}^{\star}$ derived. Each MT $n$ calculates the partial derivative of $g_{n}\left(x_{m, n}, \beta_{m, n}^{\star}\right)$ in the direction of $x_{m, n}$ for all the networks. Then MT $n$ chooses the specific network $m^{\prime}$ associated with the highest value, which may be written as:

$$
\begin{aligned}
& x_{m^{\prime}, n}^{\star}=1, \text { if } m^{\prime}=\arg \max _{\forall m} \frac{\partial g_{n}\left(x_{m, n}, \beta_{m, n}^{\star}\right)}{\partial x_{m, n}} ; \\
& x_{m, n}^{\star}=0, \text { if } m \neq m^{\prime} .
\end{aligned}
$$

At the higher level, we have the master dual problem, which may be expressed as:

$$
\min _{\boldsymbol{\lambda}, \boldsymbol{\mu}} g(\boldsymbol{\lambda}, \boldsymbol{\mu})
$$


TABLE III

Decentralized Algorithm for Problem 2

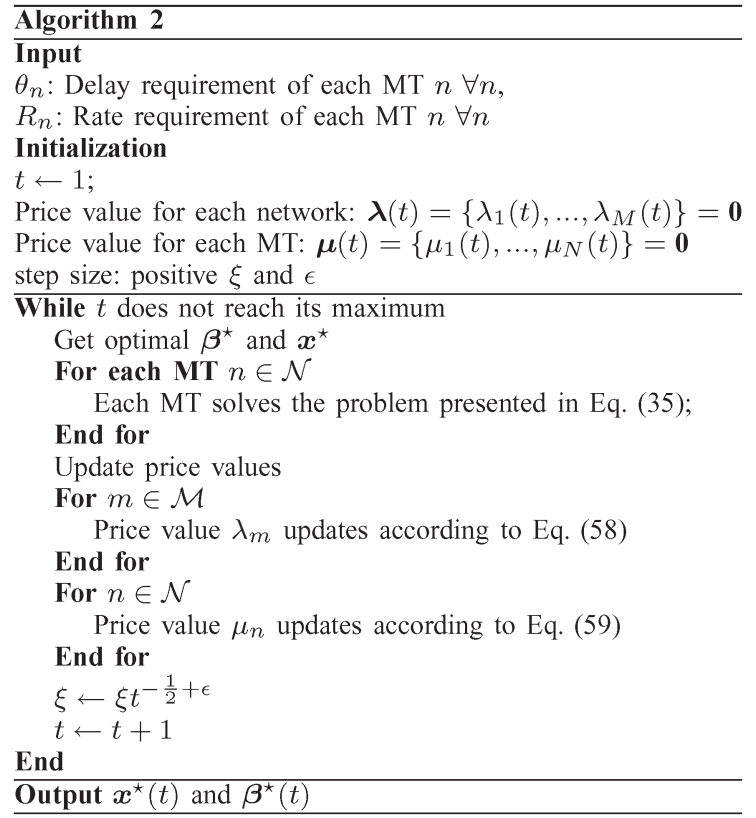

where we have $g(\boldsymbol{\lambda}, \boldsymbol{\mu})=\sum_{n \in \mathcal{N}} g_{n}\left(x_{m, n}^{\star}, \beta_{m, n}^{\star}\right)+\sum_{m \in \mathcal{M}} \lambda_{m}-$ $\sum_{n \in \mathcal{N}} \mu_{n} R_{n}$, and $x_{m, n}^{\star}, \beta_{m, n}^{\star}$ denotes the optimal value derived from the lower level optimization problem (53). Similar to the solution provided in Section IV-A, the price parameters $\lambda$ and $\boldsymbol{\mu}$ are updated according to:

$$
\begin{aligned}
\lambda_{m}(t+1) & =\left[\lambda_{m}(t)-\xi_{\lambda}(t)\left(1-\sum_{n \in \mathcal{N}} \beta_{m, n}^{\star}\right)\right]^{+} \\
\mu_{n}(t+1) & =\left[\mu_{n}(t)-\xi_{\mu}(t)\left(x_{m, n}^{\star} \sum_{n \in \mathcal{N}} \Delta_{m, n}\left(\beta_{m, n}^{\star}\right)-R_{n}\right)\right]^{+} .
\end{aligned}
$$

Similarly, our decentralized optimal RA algorithm is an iterative algorithm, which performs an optimal network selection and finds the corresponding resource block probability from network $m$ to MT $n$ based on the update of the price parameters $\lambda_{m}$ and $\mu_{n}$, over a number of iterations, until the optimal solution is reached. Each of the networks $m$ starts with an feasible initial price value $\lambda_{m}$, while each MT $n$ starts with an feasible initial price value $\mu_{n}$. Each MT broadcasts its price value to all networks. Then each network calculates the optimal network selection $x_{m, n}^{\star}$ and transmission resource block probability $\beta_{m, n}^{\star}$ based on the price information $(\boldsymbol{\lambda}, \boldsymbol{\mu})$. Each network $m$ updates its price value $\lambda_{m}$ and then broadcasts the optimal transmission resource block to the MTs. Similarly, each MT updates its price values $\mu_{n}$. The MTs broadcast their new price values $\boldsymbol{\mu}$ to the networks and the process continues, until the algorithm converges. The decentralized optimal RA algorithm is formally described in Table III.
TABLE IV

\begin{tabular}{|c|c|}
\hline \multicolumn{2}{|l|}{ 2D indoor scenario } \\
\hline Length of room & $20 \mathrm{~m}$ \\
\hline Height of room & $3 \mathrm{~m}$ \\
\hline Number of indoor MT & 10 \\
\hline Height of MT & $0.85 \mathrm{~m}$ \\
\hline Number of rate guaranteed MT & 4 \\
\hline BER target of MTs & $10^{-5}$ \\
\hline Guaranteed effective capacity & 10/20 (Mbit/s) \\
\hline Number of iteration & 100 \\
\hline \multicolumn{2}{|l|}{ VLC System } \\
\hline Height of LED & $2.5[\mathrm{~m}]$ \\
\hline Power of LED & $20[\mathrm{w}]$ \\
\hline Semi-angle at half power & 70 [deg.] \\
\hline Width of the field of view & 60 [deg.] \\
\hline Detector physical area of a PD & $1.0\left[\mathrm{~cm}^{2}\right]$ \\
\hline Refractive index of a lens at a PD & 1.5 \\
\hline $\mathrm{O} / \mathrm{E}$ conversion efficiency & $0.53[\mathrm{~A} / \mathrm{W}]$ \\
\hline Available bandwidth for VLC system & $10[\mathrm{MHz}]$ \\
\hline Reflectance factor & 0.8 \\
\hline Electronic charge & $1.6 \times 10^{-19}[\mathrm{C}]$ \\
\hline Background current & $5.1 \times 10^{-3}[\mathrm{~A}]$ \\
\hline Open-loop voltage gain & 10 \\
\hline Fixed capacitance of the PD per unit area & $1.12 \times 10^{-6}$ \\
\hline FET channel noise factor & 1.5 \\
\hline FET transconductance & $3 \times 10^{-2}$ \\
\hline \multicolumn{2}{|l|}{ Femtocell System } \\
\hline Position of the indoor femtocell & $(0,0)$ \\
\hline Transmission power of the femtocell BS & $0.02[\mathrm{w}]$ \\
\hline Indoor path-loss exponent & $3>$ \\
\hline Carrier frequency & $2[\mathrm{GHz}]$ \\
\hline Indoor path-loss constant & $37[\mathrm{~dB}]$ \\
\hline Available bandwidth for femtocell system & $5[\mathrm{MHz}]$ \\
\hline
\end{tabular}

Notations AND SySTEM PARAMETERS

\section{RESUlTS AND Discussions}

In this section, we present numerical performance results for characterizing the proposed RA algorithms in the context of the indoor HetNet of Fig. 1. Again, the "two-rate" transmission channel model is used for the VLC system, while the transmission channel is subject to uncorrelated Rayleigh fading with a unity average power and to propagation loss for the RF femtocell system. We assume that 10 MTs are uniformly distributed in the room and all experience the same delay exponents. Furthermore, the MTs are divided into two groups, where four guaranteed-rate MTs are in one of the groups, while no guaranteed rate is maintained for the MTs in the other group. Our main system parameters are summarized in Table IV.

\section{A. Convergence of the Distributed Iterative Algorithm}

Fig. 2 illustrates the convergence behavior of our distributed RA algorithm for both sophisticated multi-homing MTs and for the less advanced multi-mode MTs at a zero VLC blocking probability of $p=0$, in conjunction with $\theta=0.01$ and $\alpha=1$. For comparison, we use the BONMIN solver [37] in order to derive the optimal solution of our RA problem. As shown in Fig. 2, our distributed RA algorithm converges to the optimal value within 100 iterations for both the multi-homing MTs and for the multi-mode MTs. This result demonstrates that the distributed RA algorithm indeed finds the optimal RA probability for multi-homing MTs as well as the optimal RA probability and network selection for the multi-mode MTs, respectively. Observe furthermore from Fig. 2 that the objective function value for multi-homing MTs is higher than that for the 


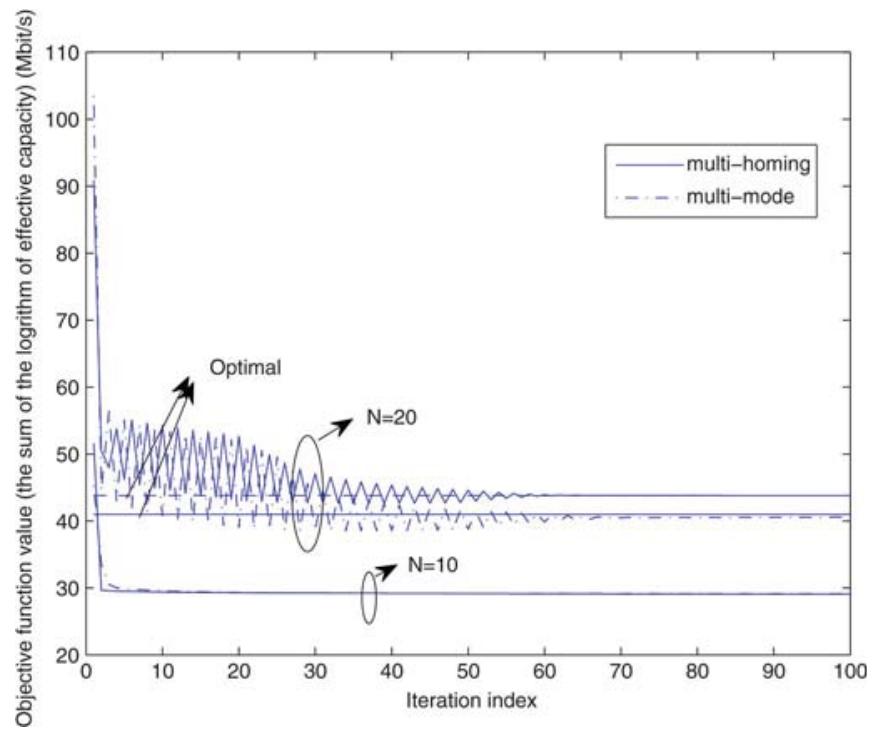

Fig. 2. Objective function (OF) values versus the total number of iterations for the proposed decentralized algorithms for both multi-homing MTs and multimode MTs, in conjunction with $\alpha=1, \theta=0.01$, and $p=0$.

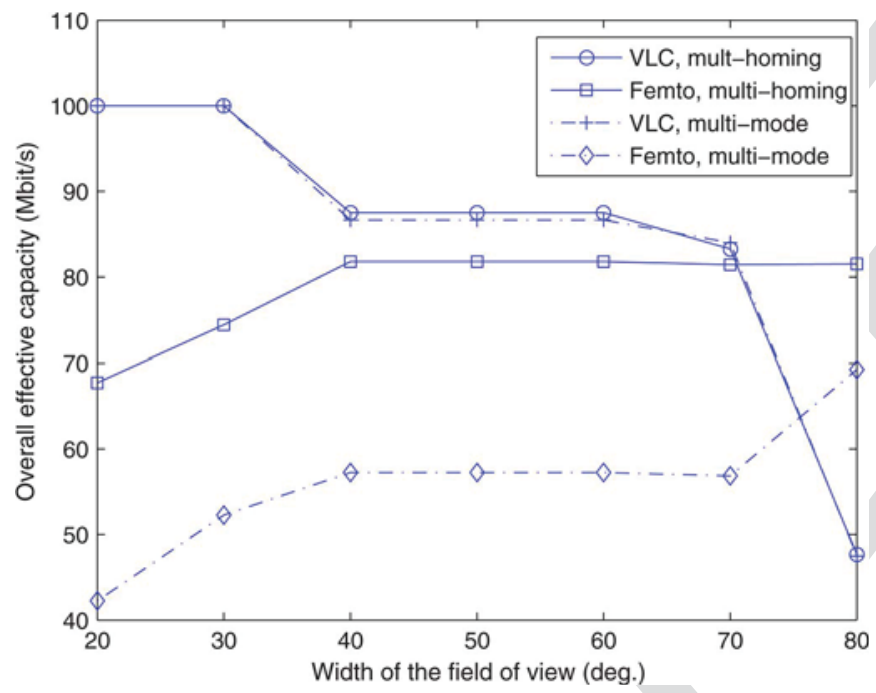

Fig. 3. The overall effective capacity of MTs versus the field of view of MTs, for $\alpha=1, \theta=1$, and $p=0$.

multi-mode MTs. This is because the RA problem of the multimode MTs might be viewed as a specific scenario of the multihoming MTs. We may also note that the speed of convergence is slower, when we increase the number of MTs.

\section{B. Effect of the Width Field of View Angle and of the Semi-Angle at Half Power}

Fig. 3 demonstrates the relationship between the FOV and the overall effective capacity of the MTs for the delay exponent of 1 and for the VLC LOS blocking probability of $p=0$. Observe from the figure that the VLC system's effective capacity is non-increasing, when we increase the angle of the FOV. This is because the gain of the optical concentrator decreases upon the increasing the FOV. We also note that the overall effective capacity of the VLC system is substantially reduced, when the FOV is larger than $70^{\circ}$. Hence in our simulation scenario the FOV of MTs should be no larger than $70^{\circ}$. Furthermore,

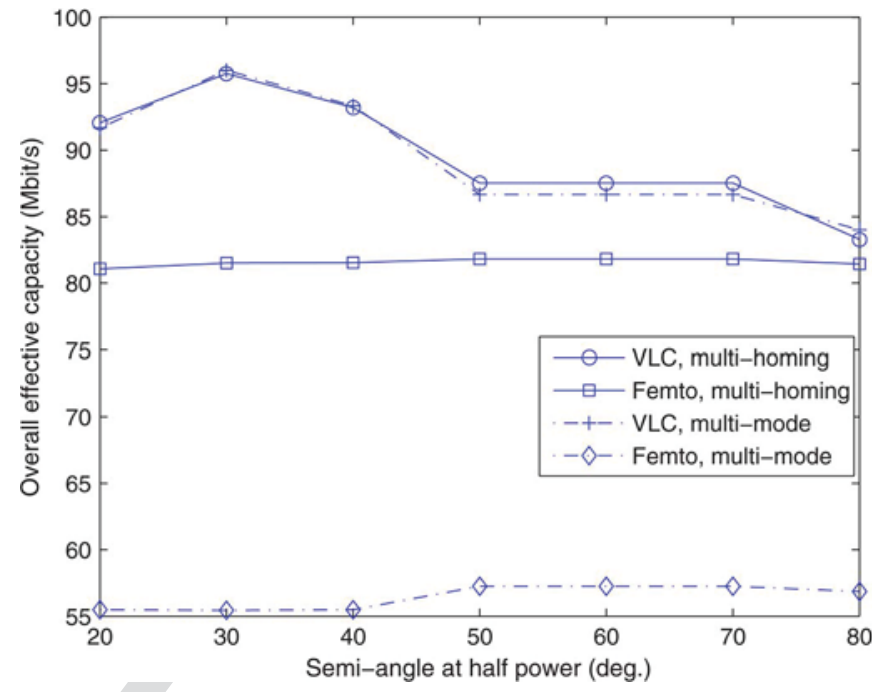

Fig. 4. The overall effective capacity of MTs versus the semi-angle at half power of the lights, for $\alpha=1, \theta=1$, and $p=0$.

it is also shown that the overall effective capacity of the RF femtocell system increases slightly. The reason might be that the specific MTs that suffer from the performance degradation of the VLC system may be compensated by having a good channel quality for the RF femtocell system.

Fig. 4 shows the overall effective capacity of the MTs as a function of the semi-angle $\phi_{1 / 2}$ at half power of the lights, for the delay exponent of 1 and VLC LOS blocking probability of $p=0$. It is observed that the VLC system's effective capacity increases, when $\phi_{1 / 2}$ increases from $20^{\circ}$ to $30^{\circ}$. Then the effective capacity decreases, when $\phi_{1 / 2}$ increases from $30^{\circ}$ to $80^{\circ}$. This is because the value of the semi-angle at half power affects the order of Lambertian emission. As a result, the direct LOS ray and the first reflected DC gain of (1) and (5) may change according to the value of $\phi_{1 / 2}$.

\section{Effective Capacity of MTs: Multi-Homing Versus Multi-Mode MTs}

Let us now compare the performance of multi-homing MTs and multi-mode MTs for different values of $\alpha$. It is observed that the performance of the multi-homing MTs is better than that of the multi-mode MTs. Again, this is because the multimode transmission may be viewed as a specific scenario of multi-homing. Hence, the performance of multi-mode MTs may be interpreted as the lower bound of the maximum achievable capacity of multi-homing MTs. When we set $\alpha=0$, as shown in Fig. 5, the RA problem is reformulated as the maximization of the total effective capacity. Since user 5 and user 6 are located in the middle of the room and are capable of achieving a better performance for the RF femtocell, the femtocell system is willing to allocate more resources to these two MTs in the multi-homing scenario.

Furthermore, owing to the rate constraint, the guaranteedrate MTs always satisfy the minimum rate requirement. For the multi-homing scenario, the VLC system and the RF femtocell system may simultaneously transmit to MT 4 in order to satisfy the rate requirement. However, for the multi-mode scenario, the femtocell system may allocate the resource to MT 4 in order 

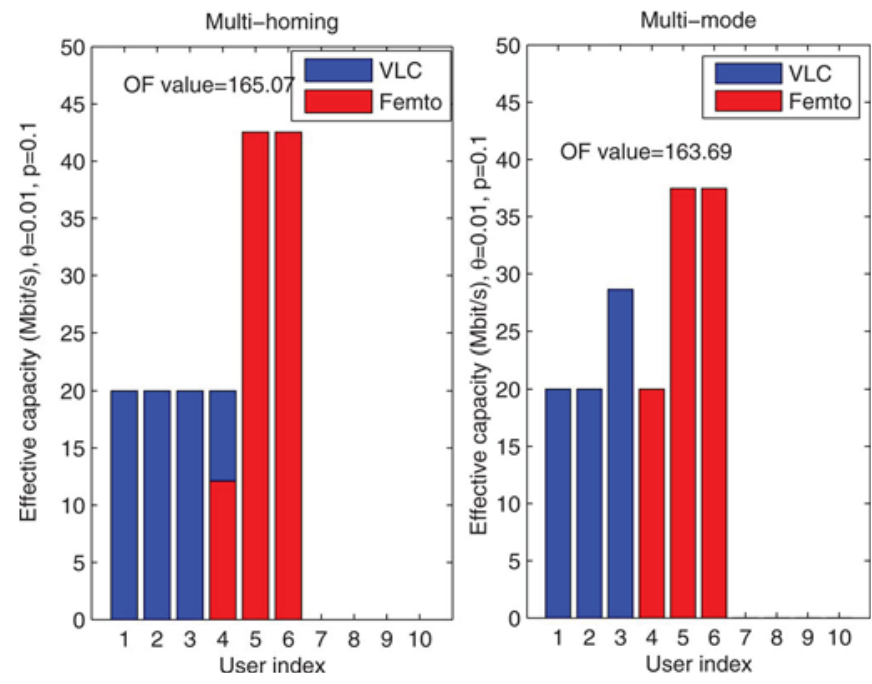

Fig. 5. The effective capacity of both multi-homing MTs and multi-mode MTs, along with $\alpha=0, \theta=0.01$, and $p=0.1$.
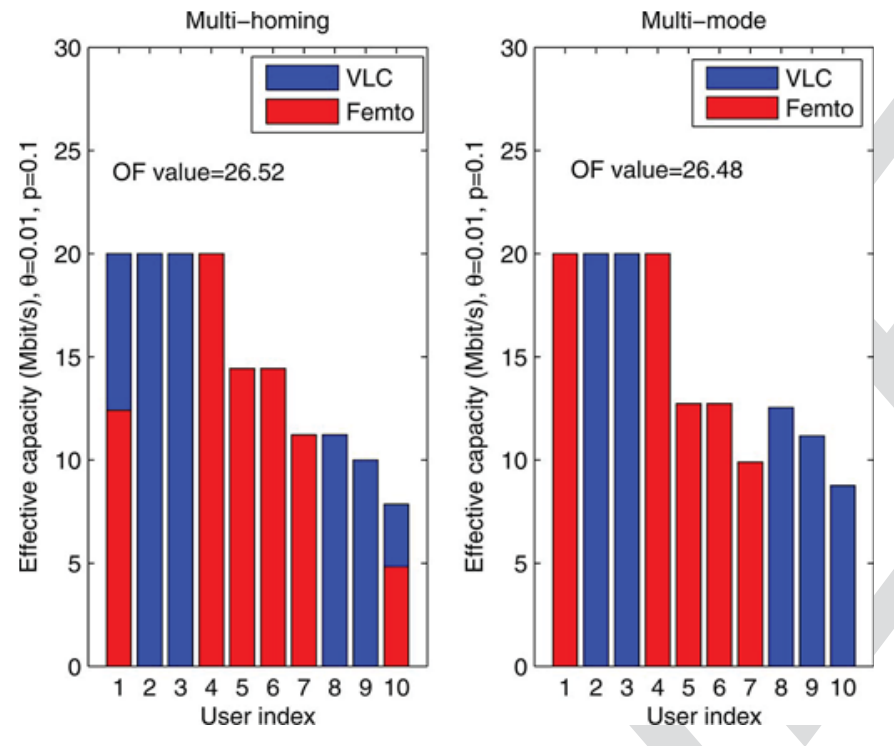

Fig. 6. The effective capacity of both multi-homing MTs and multi-mode MTs, with $\alpha=1, \theta=0.01$, and $p=0.1$.

to satisfy its rate requirement This is because the VLC system may not be able to simultaneously satisfy all of the four MTs' rate requirement. For the non-rate-guaranteed MTs, no resource will be allocated, when $\alpha=0$. This is because the VLC system prefers to allocate resources to the guaranteed-rate MTs, and the femtocell system may allocate resources to the centrally located MTs, in order to achieve the maximum total effective capacity.

When we set $\alpha=1$, observe in Fig. 6 that the RA problems are formulated under the proportional fairness constraint. It is illustrated that the objective function value for MT-homing MTs is higher than that for MT-mode MTs. The rate guaranteed MTs have higher priority and their rate requirements are satisfied for both the multi-homing and the multi-mode scenarios. It may be observed in Fig. 6 that the MTs located in the middle of the room may achieve a higher effective capacity for multihoming MTs. This is because the MTs in the middle of the room are capable of achieving a better performance, when communicating with the RF femtocell system. In contrast to
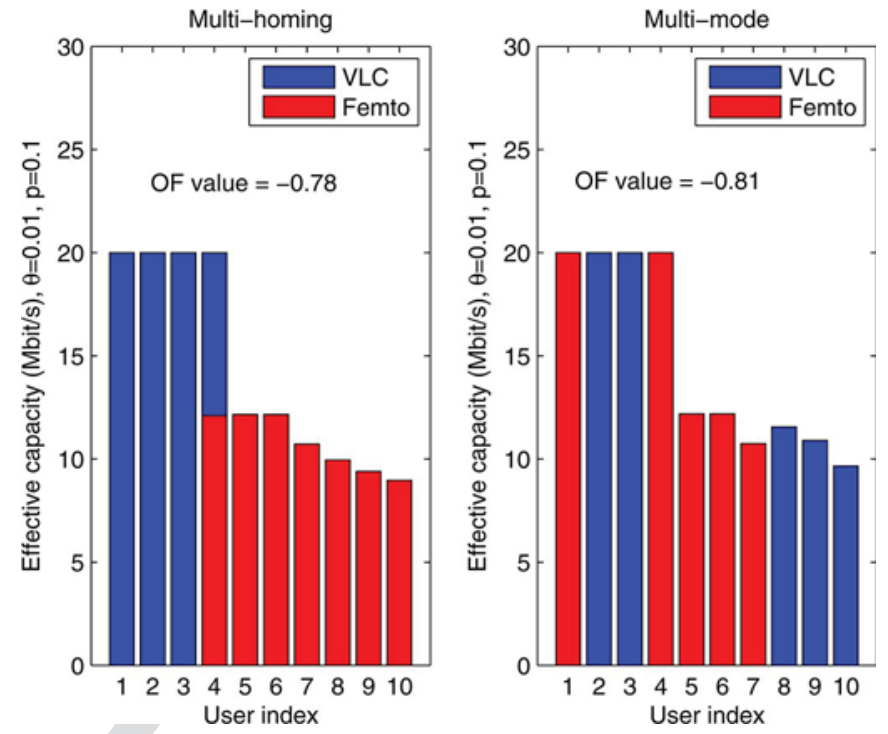

Fig. 7. The effective capacity of both multi-homing MTs and multi-mode MTs, with $\alpha=2, \theta=0.01$, and $p=0.1$.

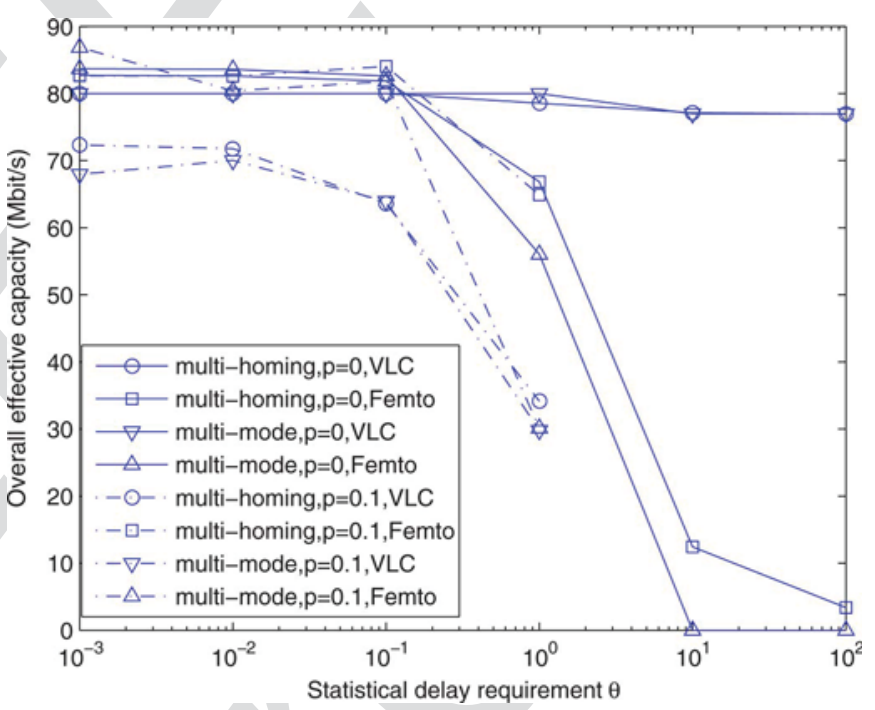

Fig. 8. The overall effective capacity of MTs versus the statistical delay parameter $\theta$, for $\alpha=1$.

the scenario of $\alpha=0$, the system may allocate resources to the non-guaranteed-rate MTs under the consideration of fairness to all MTs. Hence MT 7, 8, 9, 10 also receive their signals from the VLC system or from the femtocell system. When the MTs are of the multi-mode type, the center MTs may choose the RF femtocell system for their transmission, while the VLC system may transmit to the edge MTs. Furthermore, observe in Fig. 7 that when we increase the value of $\alpha$, the differences of effective capacity upon receiving from a specific network between the different MTs are smaller than that when we set $\alpha=1$. As a result, we believe that having a higher $\alpha$ results in a higher grade of fairness.

\section{Effect of the Delay Statistics}

Fig. 8 illustrates the effect of the delay exponent $\theta$ on the overall effective capacity of the MTs for the blocking 
probabilities of $p=0$ and $p=0.1$, respectively. Observe from the figure that the overall effective capacity is reduced upon increasing the delay exponent $\theta$. However, the overall effective capacity of both the VLC and of the RF femtocell system is fairly insensitive to the delay exponent, when the exponent is relatively small. This is because when the delay exponent is low, the resultant delay requirement is loose and the overall effective capacity is close to the Shannon capacity, which depends on the wireless channel, but is independent of the delay of the packetarrival process. However, the overall effective capacity of the RF femtocell decreases substantially, when the delay exponent is increased. Furthermore, when the delay exponent is relatively high, the overall effective capacity tends to zero. However, it is shown in Fig. 8 that the overall effective capacity of the VLC remains almost unchanged for the entire delay exponent region considered for $p=0$. The DL effective capacity between the VLC LEDs and MT $n$ is expressed as: $\Delta_{1, n}\left(\beta_{1, n}\right)=\beta_{1, n} \mathcal{R}_{1, n}$, which is independent of the delay exponent $\theta$. Observe from Fig. 8 that the effective capacity gleaned from the VLC system decreases only slightly, while the effective capacity of the RF femtocell decreases rapidly. This is because the VLC system increases the amount of resources allocated to the guaranteedthroughput MTs, at the cost of decreasing the overall effective capacity. Hence, the VLC system benefiting from a zero blocking probability is more reliable than the RF femtocell link, when the MTs have to satisfy a certain delay constraint.

When we assume that the blocking probability of the VLC system equals to 0.1 , its performance degrades rapidly for $\theta \geq 0.1$. Hence naturally, the VLC system is more sensitive to the delay constraints in the presence of a non-zero blocking probability. As a result, the RF femtocell has to increase its RA probability, especially to the MTs operating under a strict bit rate guarantee. When we have $\theta>1$, it is observed in Fig. 8 that our system fails to fulfil the bit rate requirement. In this case, no optimal solution can be found. It is also observed in Fig. 8 that the attainable performance is similar for multi-homing MTs and multi-mode MTs, when the delay requirement is loose.

\section{E. Effect of the VLC System's Blocking Probability}

Let us now quantify the effect of the VLC system's blocking probability on the overall effective capacity of the MTs, when using a delay exponent of 1. It is illustrated in Fig. 9 that the VLC system's effective capacity decreases, when the blocking probability is increased. However, as seen in Fig. 9, the effective capacity of the RF femtocell system is slightly reduced for the multi-homing MTs. The RF femtocell system should increase the amount of resources allocated to the MTs, if they have to satisfy a certain guaranteed throughput, which is achieved at the price of decreasing the overall effective capacity. When the MTs are multi-mode terminals, we observe that the overall effective capacity of the VLC system and RF femtocell system substantially decreases at $p=0.1$. This is because when the VLC LOS reception is blocked with a certain probability, the effective capacity is substantially reduced according to (18) and the first reflected ray may only contribute to the MTs located near the wall. As a result, the RF femtocell system may have to allocate resources to the guaranteed-rate MTs,

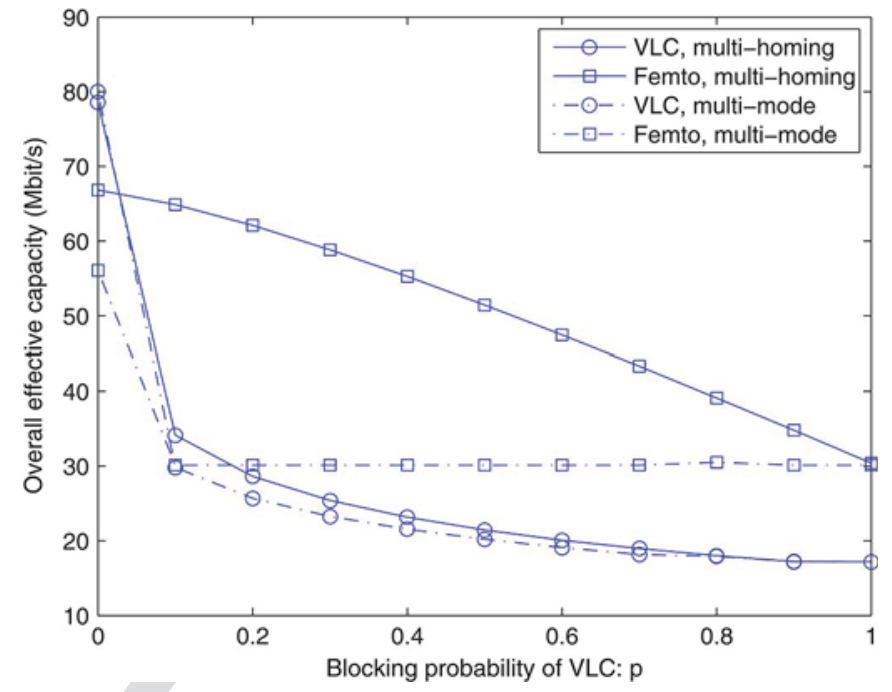

Fig. 9. The overall effective capacity of MTs versus the blocking probability of the VLC system $p$, for $\alpha=1$ and $\theta=1$.

even if their RF channel quality is not particularly good. When the LOS reception blocking probability increases from $p=0.1$ to $p=1$, the overall effective capacity of the RF femtocell system remains constant. This is because the femtocell system may allocate most of its resources to the guaranteed-rate MTs. Increasing $p$ results in decreasing the performance of the VLC system. As a result, the overall effective capacity of the VLC system keeps on decreasing. When $p=1$, the LOS ray is blocked, hence the MTs may only receive a reduced optical power due to the first reflected ray. Hence, it is plausible that when the blocking probability of the VLC system is high, the $\mathrm{RF}$ femtocell system becomes more reliable.

\section{F. Effect of the User Distribution}

In the above simulations, we assume that the MTs are uniformly located in the room. Fig. 10 compares the overall effective capacity, when all the MTs are located in the center (center cluster) or at the edge (edge cluster). Observe that the performance of the RF femtocell system of center-cluster MTs is better than that of the edge-cluster MTs. This is because we assume that the RF femtocell BS is positioned in the center of the room. As a result, the center-cluster MTs benefit from a shorter transmission distance and a lower pathloss. We also observe that the overall effective capacity of the VLC system decreases upon increasing the VLC LOS reception blocking probability $p$. When the MTs are center-clustered, the effective capacity of the VLC system is substantially reduced, as $p$ increases from 0 to 0.1 . Then it starts to decay toward 0 for larger $p$ values. The performance of the VLC system is sensitive to the LOS reception blocking, especially when the MTs are located in the center of the room. However, when the MTs are edge-clustered, the VLC system may still be capable of supporting an approximately $30 \mathrm{Mbit} / \mathrm{s}$ transmission rate, even if the VLC LOS blocking probability becomes 1 , because the edge MTs may still benefit from the reflected optical power. 

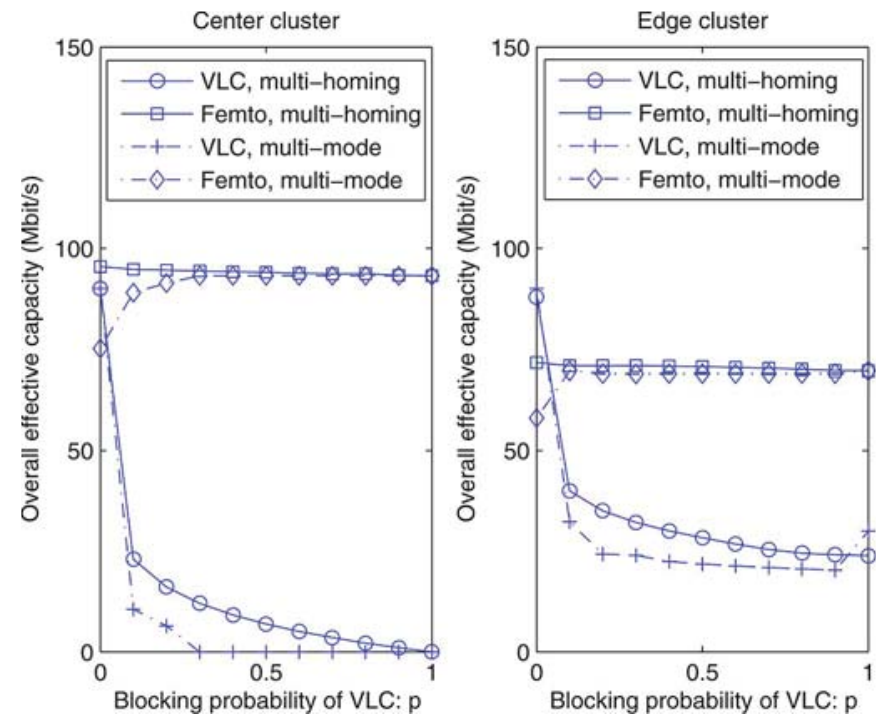

Fig. 10. The overall effective capacity of MTs versus the blocking probability of $p$, when the MTs are located at the room-center and room-edge, for $\alpha=1$ and $\theta=1$.

\section{CONCLUSION}

In this paper, we studied the resource allocation problems of mobile terminals (MTs) in a HetNet under diverse QoS requirements in terms of the data rate, fairness to MTs and the statistical delay requirements, where a hybrid VLC and RF femtocell system was considered. The objective functions relied upon were proven to be concave. Then we proposed decentralized algorithms for solving the associated RA problem. The optimal RA for each iteration of the dual decomposition algorithm were presented and simulations were performed for validating the algorithm.

Our simulation results compared the performance of the multi-homing MTs and of the multi-mode MTs in conjunction with different values of $\alpha$. Multi-homing MTs are capable of achieving a better performance than multi-mode MTs. Furthermore, our simulation results illustrated that when the VLC LOS blocking probability is zero, the VLC system is more reliable than the RF femtocell link, even if the statistical delay constraints are tight. However, it is plausible that when the VLC LOS blocking probability is high, the RF femtocell system becomes more reliable. Additionally, a comprehensive study of the effects of various system parameters was carried out.

\section{APPENDiX A}

\section{PROOF OF LEMMA 1}

Firstly, we prove that the effective capacity $\Delta_{m, n}\left(\beta_{m, n}\right)$ of the link spanning from network $m$ to MT $n$ is a concave function of $\beta_{m, n}$. The first derivative of the effective capacity $\Delta_{1, n}\left(\beta_{1, n}\right)$ from the VLC link to MT $n$ is given by:

$$
\frac{d \Delta_{1, n}}{d \beta_{1, n}}=\frac{\mathcal{R}_{2, n} p e^{-\theta_{n} \mathcal{R}_{2, n} \beta_{1, n}}+\mathcal{R}_{1, n}(1-p) e^{-\theta_{n} \mathcal{R}_{1, n} \beta_{1, n}}}{p e^{-\theta_{n} \mathcal{R}_{2, n} \beta_{1, n}}+(1-p) e^{-\theta_{n} \mathcal{R}_{n} \beta_{1, n}}} .
$$

Then the second derivative of the effective capacity $\Delta_{1, n}$ from the VLC link to MT $n$ is given by:

$$
\begin{aligned}
\frac{d^{2} \Delta_{1, n}}{d \beta_{1, n}^{2}} & =-\frac{\left(\mathcal{R}_{1, n}-\mathcal{R}_{2, n}\right)^{2} \theta_{n} p(1-p) e^{-\theta_{n} \beta_{1, n}\left(\mathcal{R}_{1, n}+\mathcal{R}_{2, n}\right)}}{\left[p e^{-\theta_{n} \mathcal{R}_{2, n} \beta_{1, n}}+(1-p) e^{-\theta_{n} \mathcal{R}_{1, n} \beta_{1, n}}\right]^{2}} \\
& \leq 0, \text { for } \theta_{n} \geq 0,0 \leq p \leq 1 .
\end{aligned}
$$

The second derivative of the effective capacity $\Delta_{2, n}$ from the RF femtocell link to MT $n$ is given by (62), shown at the bottom of the page, where inequality $a$ holds, according to the Cauchy-Schwarz inequality property presented in [40].

Assuming that we have $f_{1}\left(\beta_{m, n}\right)=\varphi_{\alpha}\left(\sum_{m \in \mathcal{M}} \Delta_{m, n}\right)$, the second partial derivative of $f_{1}\left(\beta_{m, n}\right)$ may be written as:

$$
\begin{aligned}
\frac{\partial^{2} f_{1}\left(\beta_{m, n}\right)}{\partial \beta_{m, n}^{2}} & =\frac{\Delta_{m, n}^{\prime \prime} \sum_{m \in \mathcal{M}} \Delta_{m, n}\left(\beta_{m, n}\right)-\left(\Delta_{m, n}\right)^{2}}{\left(\sum_{m \in \mathcal{M}} \Delta_{m, n}\right)^{2}} \\
& \leq 0 .
\end{aligned}
$$

As a result, the objective function (22) of Problem 1 is a concave function with respect to $\beta_{m, n}$. The linear transformations of a concave function still constitute a function, hence the problem described by (22)-(25) is a concave problem.

\section{APPENDIX B}

\section{PROOF OF LEMMA 2}

Firstly, we assume that $f_{2}\left(x_{m, n}, \beta_{m, n}\right)=x_{m, n} \varphi_{\alpha}\left(\Delta_{m, n}\right)$. Let us now use the Hessian matrix for examining the concavity of the function $f_{2}\left(x_{m, n}, \beta_{m, n}\right)$, which is given by:

$$
\mathcal{H}\left(f_{2}\right)=\left[\begin{array}{cc}
\frac{\partial^{2} f_{2}}{\partial \beta_{m, n}^{2}} & \frac{\partial^{2} f_{2}}{\partial \beta_{m, n} \partial x_{m}, n} \\
\frac{\partial^{2} f_{2}}{\partial x_{m}, n \partial \beta_{m, n}} & \frac{\partial^{2} f_{2}}{\partial x_{m, n}^{2}},
\end{array}\right],
$$

$$
\begin{aligned}
\frac{d^{2} \Delta_{2, n}\left(\beta_{2, n}\right)}{d \beta_{2, n}^{2}}=\frac{\theta_{n} B_{f}^{2}}{\ln ^{2} 2}\left\{\frac{\left[\int_{0}^{\infty}(1+x)^{\frac{-\theta_{n} B_{f} \beta_{2, n}}{\ln 2}} e^{\frac{-x}{\Gamma}} \ln (1+x) d x\right]^{2}}{\left[\int_{0}^{\infty}(1+x)^{\frac{-\theta_{n} B_{f} \beta_{2, n}}{\ln 2}} e^{\frac{-x}{\Gamma}} d x\right]^{2}}\right. & \\
& \left.-\frac{\int_{0}^{\infty}(1+x)^{\frac{-\theta_{n} B_{f} \beta_{2, n}}{\ln 2}} e^{\frac{-x}{\Gamma}} \ln ^{2}(1+x) d x \int_{0}^{\infty}(1+x)^{\frac{-\theta_{n} B_{f} \beta_{2, n}}{\ln 2}} e^{\frac{-x}{\Gamma}} d x}{\left[\int_{0}^{\infty}(1+x)^{\frac{-\theta_{n} B_{f} \beta_{2, n}}{\ln 2}} e^{\frac{-x}{\Gamma}} d x\right]^{2}}\right\} \\
\leq 0 & \left.\quad \begin{array}{l}
a \\
\leq
\end{array}\right\}
\end{aligned}
$$


where $\frac{\partial^{2} f_{1}}{\partial \beta_{m, n}^{2}}$ and $\frac{\partial^{2} f_{1}}{\partial x_{m, n}^{2}}$ are the principle minors of the Hessian matrix. Recalling the proof of Lemma 1 in Appendix A, it may be readily seen that the effective capacity of each MT $\Delta_{m, n}$ is concave functions with respect to $\beta_{m, n}$. As a result, $\frac{\partial^{2} f_{2}}{\partial \beta_{m, n}^{2}}=$ $\frac{x_{m, n} \Delta_{m, n}^{\prime \prime}-x_{m, n}\left(\Delta_{m, n}^{\prime}\right)^{2}}{\left(\Delta_{m, n}\right)^{2}}$ is non-positive. Furthermore, we have $\frac{\partial^{2} f_{1}}{\partial x_{m, n}^{2}}=0$. Hence all the principle minors of the Hessian matrix are non-positive, and therefore the function $f_{2}\left(x_{m, n}, \beta_{m, n}\right)$ is concave with respect to the variables $x_{m, n}$ and $\beta_{m, n}$.

Similarly, we can readily show that the constraint described by (46) is concave with respect to the variables $x_{m, n}$ and $\beta_{m, n}$. The constraints described by (47)-(49) are linear. As a result, Problem 3 is a concave optimization problem.

\section{REFERENCES}

[1] R. Zhang and L. Hanzo, "Wireless cellular networks," IEEE Veh. Technol. Mag., vol. 5, no. 4, pp. 31-39, Dec. 2010.

[2] L. Hanzo et al., "Wireless myths, realities, futures: From 3G/4G to optical and quantum wireless," Proc. IEEE, vol. 100, pp. 1853-1888, May 2012.

[3] D. Tsonev, S. Videv, and H. Haas, "Light fidelity (Li-Fi): Towards alloptical networking," in Proc. SPIE, Feb. 2013, pp. 900 702-1-900 702-10.

[4] S. Brueck, "Heterogeneous networks in LTE-Advanced," in Proc. 8th Int. Symp. Wireless Commun. Syst., Aachen, Germany, Nov. 2011, pp. 171-175.

[5] F. Jin, R. Zhang, and L. Hanzo, "Frequency-swapping aided femtocells in twin-layer cellular networks relying on fractional frequency reuse," in Proc. IEEE WNCN, Paris, France, Apr. 2012, pp. 3097-3101.

[6] F. Jin, R. Zhang, and L. Hanzo, "Fractional frequency reuse aided twin-layer femtocell networks: Analysis, design and optimization," IEEE Trans. Commun., vol. 61, no. 5, pp. 2074-2085, May 2013.

[7] C. L. Tsai and Z. Xu, "Line-of-sight visible light communications with InGaN-based resonant cavity LEDs," IEEE Photon. Technol. Lett., vol. 25, no. 18, pp. 1793-1796, Sep. 2013.

[8] I. Stefan, H. Burchardt, and H. Haas, "Area spectral efficiency performance comparison between VLC and RF femtocell networks," in Proc. IEEE Int. Conf. Commun., Budapest, Hungary, Jun. 2013, pp. 3825-3829.

[9] C. Luo, H. Ji, and Y. Li, "Utility based multi-service bandwidth allocation in the 4G heterogeneous wireless access networks," in Proc. IEEE WNCN, Budapest, Hungary, Apr. 2009, pp. 1-5.

[10] M. Ismail and W. Zhuang, "A distributed multi-service resource allocation algorithm in heterogeneous wireless access medium," IEEE J. Sel. Areas Commun., vol. 30, no. 2, pp. 425-432, Feb. 2012.

[11] M. Ismail and W. Zhuang, "Decentralized radio resource allocation for single-network and multi-homing services in cooperative heterogeneous wireless access medium," IEEE Trans. Wireless Commun., vol. 11, no. 11, pp. 4085-4095, Nov. 2012.

[12] M. Ismail, A. Abdrabou, and W. Zhuang, "Cooperative decentralized resource allocation in heterogeneous wireless access medium," IEEE Trans. Wireless Commun., vol. 12, no. 2, pp. 714-724, Feb. 2013.

[13] D. Wu and R. Neigi, "Effective capacity: A wireless link model for support of quality of service," IEEE Trans. Wireless Commun., vol. 2, no. 4, pp. 630-643, Jul. 2003.

[14] M. Tao, Y. Liang, and F. Zhang, "Resource allocation for delay differentiated traffic in multiuser OFDM systems," IEEE Trans. Wireless Commun., vol. 7, no. 6, pp. 2190-2201, Jun. 2008.

[15] D. S. W. Hui, V. K. N. Lau, and H. L. Wong, "Cross-layer design for OFDMA wireless systems with heterogeneous delay requirements," IEEE Trans. Wireless Commun., no. 8, pp. 2872-2880, Aug. 2007.

[16] J. Tang and X. Zhang, "Cross-layer resource allocation over wireless relay networks for quality of service provisioning," IEEE J. Sel. Areas Commun., vol. 25, no. 4, pp. 2318-2328, May 2007.

[17] Y. Cui, V. K. N. Lau, R. Wang, H. Huang, and S. Zhang, "A survey on delay-aware resource control for wireless systems-Large deviation theory, stochastic Lyapunov, distributed stochastic learning," IEEE Trans. Inf. Theory, vol. 58, no. 3, pp. 1677-1701, Mar. 2012.

[18] N. McKeown, A. Mekkittikul, V. Anantharam, and J. Walrand, "Achieving 100 percent throughput in an input-queued switch," IEEE Trans. Commun., vol. 47, no. 8, pp. 1260-1267, Aug. 1999.
[19] L. Tassiulasa and A. Ephremides, "Dynamic sever allocation to parallel queues with randomly varying connectivity," IEEE Trans. Inf. Theory, vol. 39, no. 2, pp. 466-478, Mar. 1993.

[20] M. Uchida and J. Kurose, "An information-theoretic characterization of weighted $\alpha$-proportional fairness," in Proc. IEEE INFOCOM, Rio de Janeiro, Brazil, Apr. 2009, pp. 1053-1061.

[21] J. Mo and J. Walrand, "Fair end-to-end window-based congestion control," IEEE/ACM Trans. Netw., vol. 8, no. 5, pp. 556-567, Oct. 2000.

[22] Y. Yi and M. Chiang, "Stochastic network utility maximization: A tribute to Kelly's paper published in this journal a decade ago," Eur. Trans. Telecommun., vol. 19, no. 4, pp. 421-442, Jun. 2008.

[23] A. Sang, X. Wang, M. Madihian, and R. D. Gitlin, "A flexible downlink scheduling scheme in cellular packet data systems," IEEE Trans. Wireless Commun., vol. 5, no. 3, pp. 568-577, Mar. 2006.

[24] S. Boyd and L. Vandenberghe, Convex Optimization. Cambridge, U.K.: Cambridge Univ. Press, 2004.

[25] M. Chiang, S. H. Low, A. R. Calderbank, and J. Doyle, "Layering as optimization decomposition: A mathematical theory of network architectures," Proc. IEEE, vol. 95, no. 1, pp. 255-312, Jan. 2007.

[26] F. R. Gfeller and U. Bapst, "Wireless in-house data communication via diffuse infrared radiation," Proc. IEEE, vol. 67, no. 11, pp. 1474-1486, Nov. 1979.

[27] T. Komine and M. Nakagawa, "Fundamental analysis for visiblelight communication system using LED lights," IEEE Trans. Consum. Electron., vol. 50, no. 1, pp. 100-107, Feb. 2004.

[28] J. M. Kahn and J. R. Barry, "Wireless infrared communications," Proc. IEEE, vol. 85, no. 2, pp. 265-298, Feb. 1997.

[29] X. Qiu and K. Chawla, "On the performance of adaptive modulation in cellular systems," IEEE Trans. Commun., vol. 47, no. 6, pp. 884-895, Jun. 1999.

[30] C.-S. Chang, "Stability, queue length, delay of deterministic and stochastic queueing networks," IEEE Trans. Autom. Control, vol. 39, no. 5, pp. 913-931, May 1994.

[31] C.-S. Chang and J. A. Thomas, "Effective bandwidth in high-speed digital networks," IEEE J. Sel. Areas Commun., vol. 13, no. 6, pp. 1091-1100, Aug. 1995.

[32] J. G. Kim and M. Krunz, "Bandwidh allocation in wireless networks with guaranteed packet-loss performance," IEEE/ACM Trans. Netw., vol. 8, no. 3, pp. 337-349, Jun. 2000.

[33] J. Tang and X. Zhang, "Quality-of-service driven power and rate adaptation for multichannel communications over wireless links," IEEE Trans. Wireless Commun., vol. 6, no. 12, pp. 4349-4360, Dec. 2007.

[34] I. S. Gradshteyn and I. M. Ryzhik, Table of Integrals, Series, Products. Amsterdam, The Netherlands: Elsevier, 2007.

[35] D. Bertsekas and R. Gallager, Data Networks. Upper Saddle River, NJ, USA: Prentice-Hall, 1987.

[36] F. P. Kelly, "Charging and rate control for elastic traffic," Eur. Trans. Telecommun., vol. 8, no. 1, pp. 33-37, Jan. 1997.

[37] P. Bonami et al., "An algorithmic framework for convex mixed integer nonlinear programs," Discret Optim., vol. 5, no. 2, pp. 186-204, May 2008.

[38] D. P. Palomar and M. Chiang, "A tutorial on decomposition methods for network utility maximization," IEEE J. Sel. Areas Commun., vol. 24, no. 8, pp. 1439-1451, Aug. 2006.

[39] W. Yu and R. Lui, "Dual methods for nonconvex spectrum optimization of multicarrier systems," IEEE Trans. Commun., vol. 54, no. 7, pp. 13101322, Jul. 2006.

[40] J. M. Steele, The Cauchy-Schwarz Master Class. Washington, DC, USA: Math. Assoc. Amer., 2004.

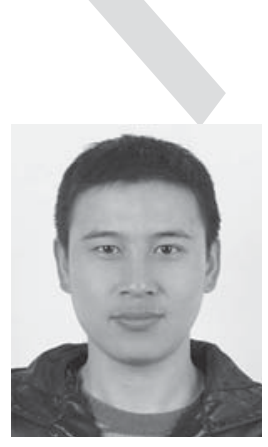

Fan Jin received the B.Eng. and M.Sc. degrees in electronics and information engineering from Huazhong University of Science and Technology, Wuhan, China, in 2008 and 2010, respectively. He is currently working toward the Ph.D. degree with the Communications Group, School of Electronics and Computer Science, University of Southampton, Southampton, U.K.

His research interests include multi-user communications, visible light communication systems, radio resource allocation, spectrum sensing, and interference management in femtocells and heterogeneous networks. He was a recipient of a scholarship under the U.K.-China Scholarships for Excellence Programme. 


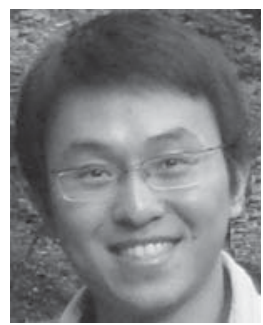

Rong Zhang (M'09) received the B.Sc. degree from Southeast University, Nanjing, China, in 2003 and the $\mathrm{Ph} . \mathrm{D}$. degree from the University of Southampton, Southampton, U.K., in 2009. Before obtaining the doctorate, he was an Engineer (August 2003-July 2004) at China Telecom and a Research Assistant (January 2006-May 2009) at Mobile Virtual Center of Excellence (MVCE), U.K. After being a Postdoctoral Researcher (August 2009-July 2012) at the University of Southampton, he took an industrial consulting leave (August 2012-January 2013) for Huawei Sweden R\&D as a System Algorithms Specialist. Since February 2013, he is a Lecturer with the CSPC Group, School of Electronics and Computer Science, University of Southampton. He has more than 40 journals in prestigious publication avenues (e.g., IEEE and OSA) and many more in major conference proceedings. He regularly serves as a Reviewer for IEEE transactions and journals and has served several times as a TPC Member/Invited Session Chair of major conferences. He was the recipient of joint funding from MVCE and EPSRC and is also a Visiting Researcher under the Worldwide University Network (WUN). More details can be found at http://www.ecs. soton.ac.uk/people/rz.

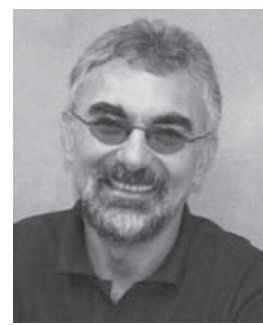

Lajos Hanzo(M'91-SM'92-F'04) received the M.S. degree in electronics and the Ph.D. degree from the Technical University of Budapest, Budapest, Hungary, in 1976 and 1983, respectively; the D.Sc. degree from the University of Southampton, Southampton, U.K., in 2004; and the "Doctor Honoris Causa" degree from the Technical University of Budapest in 2009. During his 35-year career in telecommunications, he has held various research and academic posts in Hungary, Germany, and the U.K. Since 1986, he has been with the School of Electronics and Computer Science, University of Southampton, where he holds the Chair in Telecommunications. He is currently directing a 100-strong academic research team, working on a range of research projects in the field of wireless multimedia communications sponsored by industry, the Engineering and Physical Sciences Research Council U.K., the European IST Programme, and the Mobile Virtual Centre of Excellence, U.K. During 2008-2012, he was a Chaired Professor with Tsinghua University, Beijing China. He is an enthusiastic supporter of industrial and academic liaison and offers a range of industrial courses. He has successfully supervised more than $80 \mathrm{Ph} . D$. students, coauthored 20 John Wiley/IEEE Press books on mobile radio communications totaling in excess of 10000 pages, and published 1300 research entries at IEEE Xplore. His research is funded by the European Research Council's Senior Research Fellow Grant. Dr. Hanzo is a Fellow of the Royal Academy of Engineering, the Institution of Engineering and Technology, and the European Association for Signal Processing. He is also a Governor of the IEEE Vehicular Technology Society. During 2008-2012, he was the Editor-in-Chief of the IEEE Press. He has served as the Technical Program Committee Chair and the General Chair of IEEE conferences, has presented keynote lectures, and has been awarded a number of distinctions. For further information on research in progress and associated publications, please refer to http://www-mobile. ecs.soton.ac.uk. 


\title{
Resource Allocation Under Delay-Guarantee Constraints for Heterogeneous Visible-Light and RF Femtocell
}

\author{
Fan Jin, Rong Zhang, Member, IEEE, and Lajos Hanzo, Fellow, IEEE
}

\begin{abstract}
The resource-allocation (RA) problems of mobile terminals (MTs) are investigated in a heterogeneous wireless network, where both a visible light communication system and an RF femtocell system are deployed in a room. We consider diverse quality-of-service requirements in terms of the data rate, fairness, and statistical delay requirements. Inspired by the concept of effective capacity, we formulate our optimization problems applying $\alpha$-proportional fairness while satisfying specific statistical delay constraints. Two types of MTs, namely, multihoming MTs and multimode MTs, are considered, where multihoming MTs have the capability of aggregating resources from different networks, whereas the multimode MTs always select a single network for their connection. Our optimization procedure solves the RA probability problem for multihoming MTs with the aid of a decentralized algorithm. By contrast, our optimization problem involves both network selection and RA probability optimization for multimode MTs, which may be regarded as a mixed-integer nonlinear problem. Since this problem is computationally intractable, a suboptimal decentralized method is proposed for solving it. Simulation results are also presented for clarifying the performance of the proposed algorithm. It is shown that the multimode MTs are capable of achieving similar performance to that of the multihoming MTs when the statistical delay requirements are loose. However, as expected, the multihoming MTs attain a better performance when we tighten the delay requirements.
\end{abstract}

Index Terms-Resource allocation, heterogeneous networks, VLC system, femtocell system, effective capacity.

\section{INTRODUCTION}

$\mathbf{N}$ EXT-GENERATION wireless networks are expected to be heterogeneous, integrating diverse radio access technologies (RAT) such as UMTS Terrestrial Radio Access Networks (UTRAN), GSM/EDGE Radio Access and Network (GERAN) [1], Wireless Local Area Network (WLAN) as well as millimeter-wave and possibly Visible Light Communications (VLC) networks [2], [3]. All the above networks have a coverage area overlapping with one another, hence forming a

Manuscript received February 12, 2014; revised July 17, 2014 and September 24, 2014; accepted October 10, 2014. This work was supported in part by the European Research Council under the Advanced Fellow Grant, by the RC-U.K. under the auspices of the India-U.K. Advanced Technology Centre, and by the EU's Concerto Project. The associate editor coordinating the review of this paper and approving it for publication was S. Valaee.

The authors are with the School of Electronics and Computer Science, University of Southampton, Southampton SO17 1BJ, U.K. (e-mail: fj1g10@ecs. soton.ac.uk; rz@ecs.soton.ac.uk; lh@ecs.soton.ac.uk).

Color versions of one or more of the figures in this paper are available online at http://ieeexplore.ieee.org.

Digital Object Identifier 10.1109/TWC.2014.2363451 hybrid network for wireless access, which is typically termed as a heterogeneous network (HetNet). A heterogeneous cellular system may consist of regular macro-cell base stations (BS) that typically transmit at a high power level and overlaid pico-cells, femtocells as well as relays, which transmit at substantially lower power levels [4].

Indoor cells may also form part of a HetNet. Femtocells have the potential of providing high-quality network access for indoor users at a low cost [5], [6]. As another recent addition to the wireless landscape, optical wireless systems using visible light exhibit several advantages, including license-free operation, immunity to electro-magnetic interference, network security and a high bandwidth potential [7]. The optical access point is referred to as an attocell in [3] However, the performance of VLC systems is degraded in the absence of line-ofsight (LOS) propagation. Hence we may combine VLC with a complementary network for providing a seamless data service. An optical attocell not only improves indoor coverage, it is able to enhance the capacity of the RF wireless networks, since it does not generate any additional interference. In this paper, we consider an indoor scenario, where a VLC system is combined with a classic RF femtocell system [8] and investigate the associated resource allocation (RA) problems.

For a HetNet, an important component of the integrated architecture is its radio resource management (RRM) mechanism. To access the Internet through a HetNet, the terminals such as laptops and cellphones are usually equipped with multiple wireless access network interfaces. There are two basic types of terminals. The conventional multi-mode mobile terminals are unable to support IP mobility or multi-homing. Hence, these multi-mode terminals typically opt for the specific network providing the best radio-coverage. By contrast, the family of more sophisticated terminals is equipped with IP-based mobility and multi-homing functionalities, which have the capability of aggregating radio resources from different networks. Numerous contributions have studied the RA problem in a HetNet scenario [9]-[12]. Multi-homing RA mechanisms are studied in [9], which allocate the radio bandwidth to different tele-traffic types based on the specific utility of the service supported. However, this required a central resource manager for controlling the RA process. On the other hand, the authors of [10]-[12] propose a distributed algorithm for solving the RA problem. However, these contributions only focus on maximizing the data rate, while the quality-of-service (QoS) requirements formulated in terms of the delay requirements of mobile terminals were not 
considered in these contributions. Furthermore, these contributions considered a pure RF HetNet model, while our paper considered the RA problem of a combined VLC and RF Femtocell HetNet.

Future broadband wireless networks are expected to support a wide variety of communication services having diverse QoS requirements. Applications such as voice transmission as well as real-time lip-synchronized video streaming are delaysensitive and they require a minimum guaranteed throughput. On the other hand, applications such as file transfer and email services are relatively delay-tolerant. As a result, it is important to consider the delay as a performance metrics in addition to the classic Physical (PHY) layer performance metric in crosslayer optimization. Diverse approaches may be conceived for delay-aware resource control in wireless networks [13]-[19]. For example, the average delay-constraint may be converted into an equivalent average rate-constraint using the large deviation theory approach of [13]-[17]. In order to satisfy the QoS requirements, we apply the effective capacity [13] based approach for deriving the optimal RA algorithm, which guarantees meeting the statistical delay target of HetNets.

\section{A. Main Contributions}

In this paper, we address the optimal RA in the DL of a HetNet, while meeting both the bit rate and statistical delay targets of delay-sensitive traffic. The contributions of this paper are summarized as follows:

- We consider an indoor scenario, where a combined VLC attocell and RF femtocell system is employed for providing indoor coverage. For a given position of the mobile terminal (MT), the received optical power of a MT is constituted by the sum of the direct LOS optical power and of the first reflected optical power. Hence the resultant VLC channel has two different rates, a higher one for the non-blocked LOS channel-scenario, and a reduced rate in the blocked LOS channel-scenario, when only the reflected ray is received. The RF channel is modeled as a classic Rayleigh fading channel. We investigate the VLC vs. RF activation and RA problem in this HetNet scenario.

- A limited-delay RA problem is formulated for the indoor HetNet considered. In contrast to the RA solutions of [11], [12], [15], [16], we apply the effective capacity approach of [13] for converting the statistical delay constraints into equivalent average rate constraints. Furthermore, we formulate our fairness-problem as an $\alpha$-proportional fairness utility function, as defined in [20]-[23].

- Our RA problem is formulated as a non-linear programming (NLP) problem for the multi-homing MTs. We show mathematically that this NLP problem is concave with respect to the RA probability matrix $\boldsymbol{\beta}$, which hence can be solved by convex optimization techniques, such as the barrier method of [24]. A distributed algorithm using the dual decomposition approach of [25] is proposed for RA in HetNets. It is demonstrated that this distributed algorithm approaches the optimal solution within a low number of iterations, where the optimal solution is that found by a centralized controller.
TABLE I

The Main Symbol Notations

\begin{tabular}{|c|c|}
\hline \multicolumn{2}{|r|}{ Symbol definition } \\
\hline $\mathcal{M}$ & The network set \\
\hline $\mathcal{N}$ & The mobile terminal (MT) set \\
\hline$x_{m, n}$ & The network assignment index from network $m$ to MT $n$ \\
\hline$\beta_{m, n}$ & The instantaneous probability that network $m$ transmits to MT $n$ \\
\hline$r_{m, n}$ & The instantaneous transmission bit rate \\
\hline$\psi_{i r}$ & Angle of irradiation of LED lights \\
\hline$\psi_{\text {in }}$ & Angle of incidence \\
\hline$D_{d}$ & Distance between a transmitter and a receiver \\
\hline$l$ & The order of Lambertian emission \\
\hline$\phi_{1 / 2}$ & The semi-angle at half illuminant of an LED \\
\hline$A$ & The physical area of the detector in a PD \\
\hline$T_{s}$ & The gain of an optical filter \\
\hline$g$ & The gain of an optical concentrator \\
\hline$\Psi_{c}$ & The width of the field of vision at a receiver \\
\hline$\kappa$ & A random variable with the Bernoulli distribution \\
\hline$P_{t}$ & The transmitted optical power \\
\hline$P_{r}$ & The received optical power \\
\hline$D_{1}$ & The distance between an LED light and a reflecting surface \\
\hline$D_{2}$ & The distance between a reflective point and a receiver \\
\hline$\rho$ & The reflectance factor \\
\hline$d A_{\text {wall }}$ & Reflective area \\
\hline$\omega_{1}$ & The angle of irradiance to a reflective point \\
\hline & The angle of irradiance to a MT's receiver \\
\hline$\sigma_{\text {shot }}^{2}$ & The variance of the short noise \\
\hline$\sigma_{\text {thermal }}^{2}$ & The variance of the thermal noise \\
\hline$q$ & The electronic charge \\
\hline & The detector's responsivity \\
\hline$B$ & The equivalent noise bandwidth \\
\hline$I_{b g}$ & The background current \\
\hline$\varrho$ & The Boltzmann's constant \\
\hline$T_{K}$ & The absolute temperature \\
\hline$G$ & The open-loop voltage gain \\
\hline$g_{m}$ & The FET transconductance \\
\hline$Z$ & The number of constellation points \\
\hline$\iota$ & The path-loss exponent \\
\hline$P_{f}$ & The transmitted RF power \\
\hline$\Pi$ & The recived SNR discrepancy \\
\hline$B_{f}$ & The bandwidth of the RF femtocell \\
\hline$f_{c}$ & RF carrier frequency \\
\hline$h$ & RF Rayleigh-distributed channel gain \\
\hline$\sigma^{2}$ & The variance of the AWGN \\
\hline$\theta$ & Delay-rated QoS statistical paramenter \\
\hline $\mathcal{R}_{1, n}$ & The achievable rate with VLC LOS reception \\
\hline $\mathcal{R}_{2, n}$ & The achievable rate without VLC LOS reception \\
\hline$\lambda$ & The Lagrange multiplier \\
\hline$\mu$ & The Lagrange multiplier \\
\hline$\xi$ & The step-size in the iteration algorithm \\
\hline
\end{tabular}

- We also demonstrate that the RA problem is formulated as a mixed-integer non-linear programming (MINLP) problem for multi-mode MTs, which is mathematically intractable. In order to make the problem more tractable, a relaxation of the integer variables is introduced. Then, we formally prove that the relaxed problem is concave with respect to both the relaxed network selection matrix $\boldsymbol{x}$ and to the RA probability matrix $\boldsymbol{\beta}$. Finally, a distributed algorithm is conceived using the dual decomposition approach for RA in our HetNet for the specific family of MTs having a multi-mode capability, but no multi-homing facility.

The paper is organized as follows. In Section II, we describe our system model, including both the VLC and the RF femtocell system. The formulation of our RA problems for both multi-homing MTs and multi-mode MTs is described in Section III. The proposed distributed algorithms are outlined in Section IV. Finally, our results are provided in Section V and our conclusions are offered in Section VI. The notations used in the paper are summarized in Table I. 


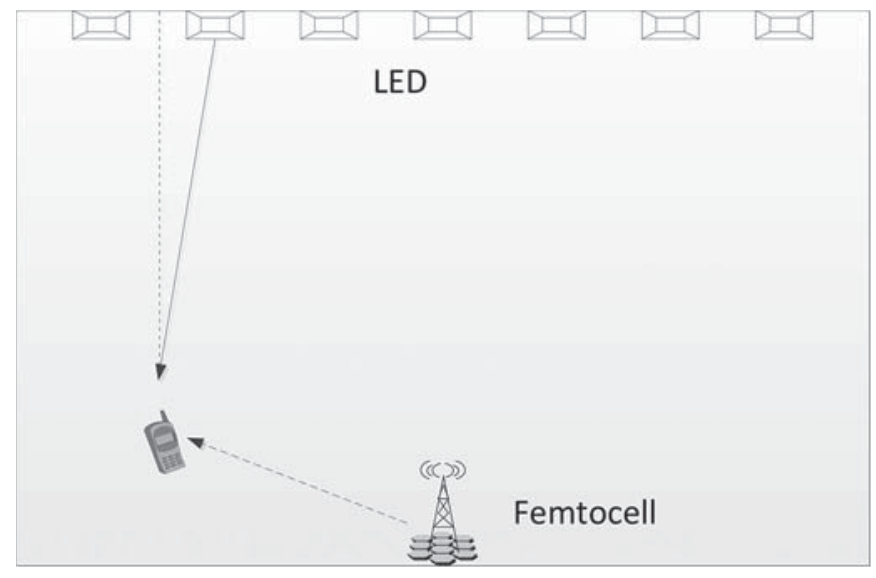

Fig. 1. The 2D model of the room using both the VLC system and the RF femtocell system.

\section{SySTEM MOdEL}

We consider a heterogeneous indoor network, where a combined VLC and a RF femtocell network are employed in a room. We denote the network set as: $\mathcal{M}=\{1,2\}$, where $m=1$ and $m=2$ represent the VLC and the RF femtocell network, respectively. It is assumed that both the VLC and the RF femtocell system are supported either by an optical fiber or powerline backbone for exchanging their roaming-related signaling information, which is unproblematic in a indoor scenario.

The set of MTs located or roaming in the indoor region is denoted by $\mathcal{N}=\{1, \cdots, n, \cdots, N\}$. We assume that the $N$ MTs are uniformly distributed in the room. Each MT for $n \in \mathcal{N}$, is capable of accessing both the VLC and the femtocell network with the same priority. Again, we consider two types of MTs, namely multi-mode MTs and multi-homing MTs. Again, the multi-mode MTs are assigned to a single network at a time, hence each multi-mode MT has to appropriately set its network selection index $x_{m, n}$, where $x_{m, n}=\{1,0\}$ indicates whether the MT $n$ is assigned to the network $m$. By contrast, for the multi-homing MTs, both the VLC and the femtocell provide resource blocks simultaneously. We denote the instantaneous transmission probability of the network $m$ transmitting to MT $n$ as $\beta_{m, n} \cdot{ }^{1}$ Furthermore, we use $\boldsymbol{x}$ and $\boldsymbol{\beta}$ to represent the network assignment matrix and RA probability matrix, respectively. The achievable throughput of MT $n$ supported by network $m$ is expressed as: $\beta_{m, n} r_{m, n}$, where $r_{m, n}$ denotes the instantaneous transmission bit rate in the DL of network $m$ to MT $n$. The VLC system has a rather different transceiver model and channel model than the traditional RF communication networks. We will now briefly introduce the achievable instantaneous transmission bit rate of both the VLC and of the RF femtocell network.

\section{A. VLC Using LED Lights}

A typical 2D-indoor VLC system using LED lights is illustrated in Fig. 1, where the LED lights are uniformly distributed on the ceiling of the room. Let us assume that $\psi_{i r}$ is the angle of

\footnotetext{
${ }^{1}$ Here $\beta_{m, n}$ may also be interpreted as the specific fraction of the normalized serving duration, during which network $m$ supports MT $n$.
}

irradiation of the LED lights, $\psi_{i n}$ is the angle of incidence, and $D_{d}$ is the distance between a transmitter and a receiver. In an optical link, the channel's direct current (DC) gain on directed path is given by [26]:

$$
H(0)= \begin{cases}\frac{(l+1) A T_{s} \kappa}{2 \pi D_{d}^{2}} \mathcal{G}, & \text { if } \psi_{\text {in }} \leq \Psi_{c}, \\ 0, & \text { if } \psi_{\text {in }}>\Psi_{c},\end{cases}
$$

where $l$ is the order of Lambertian emission, which is given by the semi-angle $\phi_{1 / 2}$ at half illumination of an LED as $l=$ $\frac{\ln 2}{\ln \left(\cos \phi_{1 / 2}\right)}$. Furthermore, $A$ is the physical area of the photodetector (PD), $T_{s}$ is the gain of the optical filter used, and $\Psi_{c}$ denotes the width of the field of view (FOV) at the receiver. Owing to the obstructions, the LOS propagation of the VLC system might be blocked. Here, the random variable $\kappa$ denotes the VLC LOS blocking event. This event is random and unpredictable. Here we assume that the event obeys the Bernoulli distribution, which indicates whether the VLC LOS reception is blocked or not. Its probability mass function may be expressed as:

$$
f(\kappa)= \begin{cases}1-p, & \text { if } \kappa=1 \\ p, & \text { if } \kappa=0\end{cases}
$$

where $p$ denotes the VLC LOS blocking probability. Equation (1) indicates that once the angle of irradiation of a receiver is higher than the Field of View (FOV), the receiver's LOS would be blocked. We assume that $\mathcal{G}=\cos ^{l}\left(\psi_{i r}\right) g\left(\psi_{\text {in }}\right) \cos \left(\psi_{\text {in }}\right)$, where $g\left(\psi_{i n}\right)$ is the gain of an optical concentrator [27] and can be characterized as [28]:

$$
g\left(\psi_{i n}\right)= \begin{cases}\frac{l^{2}}{\sin ^{2} \Psi_{c}}, & \text { if } \psi_{i n} \leq \Psi_{c} \\ 0, & \text { if } \psi_{i n}>\Psi_{c} .\end{cases}
$$

Apart from the VLC LOS reception, the MTs may also receive the VLC reflected path owing to the wall. The received optical power is given by the channel's DC gain on the direct LOS path $H_{d}(0)$ and reflected path $H_{r e f}(0)$, which may be written as:

$$
P_{r}=\sum^{\text {LEDs }}\left[P_{t} H_{d}(0)+\int_{\text {walls }} P_{t} d H_{r e f}(0)\right],
$$

where $P_{t}$ and $P_{r}$ denotes the transmitted and received optical power, respectively. The channel DC gain on the first reflection $d H_{\text {ref }}$ is given by [27]:

$$
d H_{r e f}(0)= \begin{cases}\frac{(l+1) A T_{s} \rho}{2 \pi^{2} D_{1}^{2} D_{2}^{2}} \mathcal{J} \mathcal{G} d A_{\text {wall }}, & \text { if } \psi_{\text {in }} \leq \Psi_{c}, \\ 0, & \text { if } \psi_{\text {in }}>\Psi_{c}\end{cases}
$$

where $D_{1}$ is the distance between an LED light and a reflecting surface, $D_{2}$ denotes the distance between a reflective point and a MT receiver, $\rho$ is the reflectance factor, whilst $d A_{\text {wall }}$ is a small reflective area. Furthermore, we assume that $\mathcal{J}=\cos \left(\omega_{1}\right) \cos \left(\omega_{2}\right)$, where $\omega_{1}$ and $\omega_{2}$ denote the angle of irradiance to a reflective point and the angle of irradiance to a MT's receiver, respectively.

The dominant noise contribution is assumed to be the shot noise due to ambient light from windows. We also take thermal noise into account. Hence, the receiver filter's output contains Gaussian noise having a total variance of $\sigma_{\mathrm{VLC}}^{2}$ given by the 
sum of contributions from both the shot noise and the thermal noise, which is expressed as:

$$
\sigma_{\mathrm{VLC}}^{2}=\sigma_{\text {shot }}^{2}+\sigma_{\text {thermal }}^{2}
$$

According to [27], the variance of shot noise $\sigma_{\text {shot }}^{2}$ is given by:

$$
\sigma_{\text {shot }}^{2}=2 q \varsigma P_{r} B+2 q I_{b g} I_{1} B,
$$

where $q$ is the electronic charge, $\varsigma$ is the detector's responsivity. $B$ is the equivalent noise bandwidth and $I_{b g}$ is the background current caused by the background light. Furthermore, the variance of thermal noise is given by [27]:

$$
\sigma_{\text {thermal }}^{2}=\frac{8 \pi \varrho T_{K}}{G} \eta A I_{1} B^{2}+\frac{16 \pi^{2} \varrho T_{k} \Gamma}{g_{m}} \eta^{2} A^{2} I_{2} B^{3},
$$

where $\varrho$ is the Boltzmann's constant, $T_{K}$ is the absolute temperature, $G$ is the open-loop voltage gain, $\eta$ is the fixed capacitance of the PD per unit area, $\Gamma$ is the FET channel noise factor, and finally, $g_{m}$ is the FET transconductance. The noise bandwidth factors $I_{1}$ and $I_{2}$ are constant experimental values. Here, we opt for the typical values used in the literature [27], [28], namely for $I_{1}=0.562$ and $I_{2}=0.0868$.

Let us define the receiver's SNR in the VLC system's MT $n$ as $\gamma_{1, n}$, which is given by:

$$
\gamma_{1, n}=\frac{P_{r}}{\sigma_{\mathrm{VLC}}^{2}} .
$$

We assume that the VLC system relies on M-PAM based transmission. According to [7], the relationship between the bit error ratio (BER) and SNR for M-PAM signals is approximated as:

$$
\mathrm{BER}_{\mathrm{M}-\mathrm{PAM}} \approx \frac{Z-1}{Z} \frac{2}{\log _{2} Z} Q\left(\frac{\sqrt{\gamma_{1, n} / 2}}{Z-1}\right),
$$

which $Z$ is the number of constellation points. As a result, the maximum affordable $Z$ value may be found for a given BER target. Then, the achievable transmission rate of the VLC system is given by:

$$
R=B \log _{2} Z
$$

\section{B. Channel Model of the RF Femtocell}

In this paper, we assume that the DL channel of the femtocell to a MT is subject to uncorrelated Rayleigh fading ${ }^{2}$ obeying a unity average power constraint and inverse- $\iota$ th power pathloss. Hence the instantaneous transmission bit rate of the RF femtocell is given by the classical Shannon capacity of:

$$
R=B_{f} \log _{2}\left(1+\frac{P_{f}}{\sigma^{2}} \Pi A_{f} d^{-\iota} h\right),
$$

where $B_{f}$ denotes the bandwidth of the RF femtocell, $P_{f}$ denotes the DL transmission power of the femtocell's radio port. Let us now employ continuous rate adaptation and let $\Pi$

\footnotetext{
${ }^{2}$ Since we may not be able to guarantee line of sight transmission for the $\mathrm{RF}$ femtocell system, here we consider the worst-case scenario, where the DL channel of the RF femtocell system is modeled as the widely used Rayleigh channel.
}

be the received SNR discrepancy from the continuous input memoryless channel's capacity at the target BER, which is given by: $\Pi=-\frac{1.5}{\log (5 \mathrm{BER})}$ [29]. Furthermore, $A_{f}$ is a constant that depends on the carrier frequency $f_{c}, d$ denotes the distance of this link, while $\iota$ is the pathloss exponent and finally, $h$ is the Rayleigh-distributed channel gain. Let us define the receiver's $S N R$ in the DL of the femtocell to MT $n$ as $\gamma_{2, n}$, which is given by:

$$
\gamma_{2, n}=\frac{P_{f}}{\sigma^{2}} A_{f} d^{-\iota} h,
$$

where $\sigma^{2}$ denotes the variance of the AWGN. The probability density of function (PDF) of the SNR $\gamma_{2, n}$ of a Rayleighchannel obeys the exponential-distribution of:

$$
f_{\gamma_{2, n}}(x)=\frac{1}{\Gamma} e^{-\frac{1}{\Gamma} x},
$$

where we have $\Gamma=\frac{P_{f} \Pi A_{f} d^{-\iota}}{\sigma^{2}}$.

\section{Effective Capacity}

In next-generation networks, it is essential to consider a range of QoS metrics, such as the achievable data rate, the tolerable delay and the delay-violation probability. During the early 90's, statistical QoS guarantees have been extensively studied in the context of effective bandwidth theory of [30]-[32]. The theory states that for a dynamic queuing system having stationary ergodic arrival and service processes, the probability of exceeding a certain queue length decays exponentially. Inspired by the effective bandwidth theory, the authors of [13] proposed a link-layer metric termed as the effective capacity, which characterizes the effect of delay on the system. Owing to its advantages, the effective capacity has been widely adopted [13]-[17] for studying the steady-state delay-target violation probability. According to [13], for a dynamic queuing system where the arrival and service processes are stationary and ergodic, the probability that the queue length $D(t)$ at an instant $t$ of a service exceeds the maximum tolerable delay bound $D_{\max }$ is given by:

$$
\operatorname{Pr}\left\{D(t) \geq D_{\max }\right\} \approx \gamma(t) e^{-\theta D_{\max }},
$$

where $\gamma(t)=\operatorname{Pr}\{D(t) \geq 0\}$ is the probability that the transmission queuing buffer is non-empty at a randomly selected instant $t$. Note from (15) that $\theta \geq 0$ is a crucial parameter, directly characterizing the exponential decaying rate of the probability that the delay exceeds $D_{\max }$. As a result, $\theta$ may be referred to as delay-related QoS exponent of a connection.

The effective capacity may also be interpreted as the maximum constant packet-arrival rate that the system is capable of supporting, without violating a given delay-related QoS requirement indicated by the $\mathrm{QoS}$ exponent $\theta$. For uncorrelated block fading channels wherein the service process is uncorrelated, the effective capacity is defined as [13], [33]:

$$
\Delta(\theta)=-\frac{1}{\theta} \ln \mathbb{E}[\exp (-\theta r)],
$$

where $\mathbb{E}(\cdot)$ is the expectation operator and $r$ denotes the throughput. 
1) Effective Capacity of VLC: Given the position of a MT $n$, if the angle of irradiation of a receiver is higher than the FOV, the effective capacity of VLC $\Delta_{1, n}$ is equal to 0 . However, if the angle of irradiation of a receiver is lower than the FOV, the instantaneous transmission rate $r_{1, n}$ obeys a Bernoulli distribution, with the probability mass function of:

$$
f\left(r_{1, n}\right)= \begin{cases}1-p, & \text { if } r_{1, n}=\mathcal{R}_{1, n} \\ p, & \text { if } r_{1, n}=\mathcal{R}_{2, n},\end{cases}
$$

where $\mathcal{R}_{1, n}$ and $\mathcal{R}_{2, n}$ denote the achievable transmission rate from VLC system to MT $n$ with and without VLC LOS reception, respectively. As a result, the VLC transmission channel is modeled as a two-rates channel.

Based on the above discussions, the VLC system's effective capacity, which is again the maximum constant packet-arrival rate that the service is capable of supporting under the statistical delay limit of the MT $n$ specified by $\theta_{n}$, may be expressed as:

$$
\Delta_{1, n}= \begin{cases}-\frac{1}{\theta_{n}} \ln \mathcal{Q}, & \text { if } \psi_{i n} \leq \Psi_{c}, \\ 0, & \text { if } \psi_{i n}>\Psi_{c}\end{cases}
$$

where we have $\mathcal{Q}=p e^{-\theta_{n} \beta_{1, n} \mathcal{R}_{2, n}}+(1-p) e^{-\theta_{n} \beta_{1, n} \mathcal{R}_{1, n}}$

2) Effective Capacity of the RF Femtocell: According to (12), (14), and (16), the effective capacity of the RF femtocell system to MT $n$ is given by:

$$
\begin{aligned}
\Delta_{2, n} & =-\frac{1}{\theta_{n}} \ln \int_{0}^{\infty} e^{-\theta_{n} \beta_{2, n} B_{f} \log _{2}(1+x)} f_{\gamma_{2, n}}(x) d x \\
& =-\frac{1}{\theta_{n}} \ln \left[\frac{1}{\Gamma} \int_{0}^{\infty}(1+x)^{-\frac{\theta_{n} \beta_{2, n} B_{f}}{\ln 2}} e^{-\frac{x}{\Gamma}} d x\right] \\
& =-\frac{1}{\theta_{n}} \ln \left(\Gamma^{-\frac{\theta_{n} \beta_{2, n} B_{f}}{2 \ln 2}} e^{\left.\frac{1}{2 \Gamma} \mathcal{T}\right)}\right. \\
& =\frac{\beta_{2, n} B_{f}}{2 \ln 2} \ln \Gamma-\frac{1}{2 \theta_{n} \Gamma}-\frac{1}{\theta_{n}} \ln \mathcal{T},
\end{aligned}
$$

where $\mathcal{T}=\mathcal{W}_{-} \frac{\theta_{n} \beta_{2, n} B_{f}}{2 \ln 2}, \frac{\ln 2-\theta_{n} \beta_{2, n} B_{f}}{2 \ln 2}\left(\frac{1}{\Gamma}\right)$, and $\mathcal{W}$.,. (.) represents the Whittaker functions [34].

Note from the (18) and (19), the effective capacity of the network $m$ to MT $n$ is the function of $\beta_{m, n}$. In the following sections, we will use $\Delta_{m, n}$ to represent $\Delta_{m, n}\left(\beta_{m, n}\right)$.

\section{PROBLEM FORMULATION}

In this section, the RA problem formulation is presented for both multi-homing MTs and multi-mode MTs in the indoor HetNet considered. Let us now adopt a utility function based perspective, assuming that the MT $n$ obtains utility $U_{m, n}\left(\beta_{m, n}\right)$ from network $m$.

\section{A. Utility Function: $\alpha$-Proportional Fairness Approach}

In this paper, we consider the RA problem under the consideration of the concept fairness. The mathematical concept of fairness is formulated as an optimization problems. The fairness optimization problem may be interpreted as a throughput maximization problem [35], as a max-min fairness problem [35] and as a proportional fairness problem [36]. Here a generic fairness notion referred to $\alpha$-proportional fairness [20]-[23] is introduced, which embodies a number of fairness concepts, including the above-mentioned three problems by appropriately adjusting the values of the parameter $\alpha$. We define the utility $U_{m, n}\left(\beta_{m, n}\right)$ as:

$$
U_{m, n}\left(\beta_{m, n}\right)=\varphi_{\alpha}\left(\Delta_{m, n}\right),
$$

where $\varphi_{\alpha}(\cdot)$ denotes the $\alpha$-proportional fairness defined in [21], where $\varphi_{\alpha}(\cdot)$ is a monotonically increasing, strictly concave and continuously differentiable function, which may be expressed as:

$$
\varphi_{\alpha}(x)= \begin{cases}\log (x), & \text { if } \alpha=1 \\ \frac{x^{1-\alpha}}{1-\alpha}, & \text { if } \alpha \geq 0, \alpha \neq 1 .\end{cases}
$$

We note that for different values of $\alpha$, maximizing our utility function $U_{m, n}\left(\beta_{m, n}\right)$ reduces to several well-known fairness concepts. For example, the maximum effective capacity is achieved for $\alpha=0$ [35], proportional fairness is achieved for $\alpha=1$ [36] and max-min fairness [35] is obtained, when we have $\alpha \rightarrow \infty$.

\section{B. Problem Formulation for Multi-Homing MTs}

When the MTs are capable of multi-homing, both the VLC and the RF femtocell networks may allocate resource blocks for simultaneously supporting the MTs. We set out to maximize the overall effective capacity of all indoor MTs. As a result, the corresponding RA problem may be formulated as:

$$
\begin{aligned}
\text { Problem 1: } & \underset{\boldsymbol{\beta}}{\operatorname{maximize}} \sum_{n \in \mathcal{N}} \varphi_{\alpha}\left(\sum_{m \in \mathcal{M}} \Delta_{m, n}\right) \\
\text { subject to : } & \sum_{m \in \mathcal{M}} \Delta_{m, n} \geq R_{n}, \forall n \in \mathcal{N}, \\
& \sum_{n \in \mathcal{N}} \beta_{m, n} \leq 1, \forall m \in \mathcal{M}, \\
& 0 \leq \beta_{m, n} \leq 1 .
\end{aligned}
$$

The effective capacity $\Delta_{1, n}$ of the VLC and $\Delta_{2, n}$ of the femtocell is given by (18) and (19), respectively. Physically, the constraint (23) ensures that the HetNet is capable of satisfying the bit-rate $R_{n}$ of the MT $n$, while the constraint (24) guarantees that the total transmission probability for each of the networks should always be less than 1. Finally, the constraint (25) describes the feasible region of the optimization variables.

Lemma 1: The RA problem described by (22)-(25) is a concave optimization problem.

Proof: See Appendix A.

While the Problem 1 of (22)-(25) may be solved in a centralized manner with the aid of a central resource manager, this is not a viable practical solution, when the available networks are operated by different service providers.

\section{Problem Formulation for Multi-Mode MTs}

When multi-mode MTs are considered, only a single network supports the MT at a time. Then, the RA problem may be formulated as that of maximizing the total utility $U_{m, n}\left(\beta_{m, n}\right)$ 
under the constraint of the QoS requirements expressed in terms of each MT's overall effective capacity, fairness and delay as follows:

$$
\begin{aligned}
\text { Problem 2: } & \max _{\boldsymbol{x}, \boldsymbol{\beta}} \sum_{n \in \mathcal{N}} \varphi_{\alpha}\left(\sum_{m \in \mathcal{M}} x_{m, n} \Delta_{m, n}\right) \\
\text { subject to : } & \sum_{m \in \mathcal{M}} x_{m, n} \Delta_{m, n} \geq R_{n}, \forall n \in \mathcal{N}, \\
& \sum_{n \in \mathcal{N}} x_{m, n} \beta_{m, n} \leq 1, \forall m \in \mathcal{M}, \\
& \sum_{m \in \mathcal{M}} x_{m, n}=1, \\
& x_{m, n}=\{1,0\}, \quad 0 \leq \beta_{m, n} \leq 1 .
\end{aligned}
$$

The effective capacity of VLC $\Delta_{1, n}$ and femtocell $\Delta_{2, n}$ is given by (18) and (19), respectively.

Under this formulation, the variables to be optimized are $x_{m, n}$ and $\beta_{m, n}, \forall m, n$. Physically, the constraint (27) ensures that the HetNet is capable of satisfying the bit-rate $R_{n}$ of the MT $n$, while the constraint (28) guarantees that the total transmission probability for each of the networks should always be less than 1. Furthermore, the constraint (29) guarantees that each MT should always select only one of the networks for its transmissions. Finally, the constraint (30) describes the feasible region of the optimization variables.

We note that Problem 2 is a mixed integer non-linear programming (MINLP) problem that involves both binary variables $x_{m, n}$ and real-valued positive variables $\beta_{m, n}$ during optimization. In general, MINLP problems are mathematically intractable. Nonetheless, recently several optimization tools have been developed for solving MINLP problems. The BONMIN solver [37] is for example capable of solving smooth, twice differentiable, mixed integer nonlinear programs, which was deployed for providing the upper-bound benchmark solution.

\section{Decentralized Sub-Optimal Resource ALLOCATION SCHEMES}

Problem 1 and Problem 2 may be solved with the aid of centralized optimization tools. However, in order to reduce the computational complexity and to make the problem tractable, in this section we propose decentralized sub-optimal RA schemes.

\section{A. Decentralized Solution for Multi-Homing MTs}

Since the problem is a concave one, convex duality implies that the optimal solution to this problem may be found from the Lagrangian formulation [24]. The Lagrangian function for (22) under the constraints of (23)-(25) can be expressed as:

$$
\begin{aligned}
L(\boldsymbol{\beta}, \boldsymbol{\lambda}, \boldsymbol{\mu})=\sum_{n \in \mathcal{N}}[ & \varphi_{\alpha}\left(\sum_{m \in \mathcal{M}} \Delta_{m, n}\right)-\sum_{m \in \mathcal{M}} \lambda_{m} \beta_{m, n} \\
& \left.+\sum_{m \in \mathcal{M}} \mu_{n} \Delta_{m, n}\right]+\sum_{m \in \mathcal{M}} \lambda_{m}-\sum_{n \in \mathcal{N}} \mu_{n} R_{n},
\end{aligned}
$$

where we have $\mathbf{0} \leq \boldsymbol{\beta} \leq \mathbf{1}$, while $\mu_{n}$ and $\lambda_{m}$ are the Lagrange multipliers or prices associated with the $n$th inequality constraint (23) and with the $n$th inequality constraint (24), respectively. The dual objective function $g(\boldsymbol{\lambda}, \boldsymbol{\mu})$ is defined as the maximum value of the Lagrangian over $\boldsymbol{\beta}$, which is expressed as:

$$
g(\boldsymbol{\lambda}, \boldsymbol{\mu})=\sup _{\boldsymbol{\beta}} L(\boldsymbol{\beta}, \boldsymbol{\lambda}, \boldsymbol{\mu}) .
$$

The dual variables $(\boldsymbol{\lambda}, \boldsymbol{\mu})$ are dual feasible if we have $\boldsymbol{\lambda} \geq$ $0, \boldsymbol{\mu} \geq 0$. The dual function can then be maximized for finding an upper bound on the optimal value of the original problem (22):

$$
\begin{array}{cc} 
& \min _{\boldsymbol{\lambda}, \boldsymbol{\mu}} g(\boldsymbol{\lambda}, \boldsymbol{\mu}) \\
\text { sub } \quad \boldsymbol{\lambda} \geq 0, \boldsymbol{\mu} \geq 0,
\end{array}
$$

which is always a convex optimization problem. The difference between the optimal primal objective and the optimal dual objective is referred to as the duality gap, which is always nonnegative. A central result in convex analysis showed that when the problem is convex, the duality gap reduces to zero at the optimum [24], [38]. Hence, the primal problem of (22) can be equivalently solved by solving the dual problem of (33). In principle, the dual problem may be readily solved using standard routines, such as the Newton method and the barrier method [24]. However, these algorithms generally involve centralized computation and require global knowledge of all parameters. Hence, we propose an optimal decentralized RA algorithm for solving the problem using full dual decomposition [25].

Recall that in (32) we defined a dual objective function $g_{n}\left(\lambda_{m}, \mu_{n}\right)$ for MT $n$, which may be written as:

$$
\begin{aligned}
g_{n}\left(\lambda_{m}, \mu_{n}\right)= & \varphi_{\alpha}\left(\sum_{m \in \mathcal{M}} \Delta_{m, n}\right)-\sum_{m \in \mathcal{M}} \lambda_{m} \beta_{m, n} \\
& +\sum_{m \in \mathcal{M}} \mu_{n} \Delta_{m, n} .
\end{aligned}
$$

Our primal problem described by (22)-(25) may be separated into two levels of optimization. At the lower level, we decouple the problem of (32) into $N$ subproblems, where the $n t h$ subproblem may be written as:

$$
\beta_{m, n}^{\star}=\arg \max _{0 \leq \beta_{m, n} \leq 1} g_{n}\left(\lambda_{m}, \mu_{n}\right), \forall m \in \mathcal{M} .
$$

It may be shown that $g_{n}\left(\lambda_{m}, \mu_{n}\right)$ is concave with respect to the variable $\beta_{m, n}$. Hence the maximization of $g_{n}\left(\lambda_{m}, \mu_{n}\right)$ may be achieved by finding the partial derivative of $g_{n}\left(\lambda_{m}, \mu_{n}\right)$ with respect to $\beta_{m, n}$, which is given by:

$$
\frac{\partial g_{n}\left(\lambda_{m}, \mu_{n}\right)}{\partial \beta_{m, n}}=\frac{\Delta_{m, n}^{\prime}}{\sum_{m \in \mathcal{M}} \Delta_{m, n}}+\mu_{n} \Delta_{m, n}^{\prime}-\lambda_{m},
$$


where $\Delta_{m, n}^{\prime}=\frac{d \Delta_{m, n}}{d \beta_{m, n}}$. Furthermore, the second partial derivative of $g_{n}\left(\lambda_{m}, \mu_{n}\right)$ is given by:

$$
\begin{aligned}
\frac{\partial^{2} g_{n}\left(\lambda_{m}, \mu_{n}\right)}{\partial \beta_{m, n}^{2}}= & \mu_{n} \Delta_{m, n}^{\prime \prime}+\frac{\Delta_{m, n}^{\prime \prime} \sum_{m \in \mathcal{M}} \Delta_{m, n}}{\left(\sum_{m \in \mathcal{M}} \Delta_{m, n}\right)^{2}} \\
& -\frac{\left(\Delta_{m, n}^{\prime}\right)^{2}}{\left(\sum_{m \in \mathcal{M}} \Delta_{m, n}\right)^{2}} \leq 0 \\
\frac{\partial^{2} g_{n}\left(\lambda_{m}, \mu_{n}\right)}{\partial \beta_{i, n} \partial \beta_{j, n}}= & -\frac{\Delta_{i, n}^{\prime} \Delta_{i, n}^{\prime}}{\left(\sum_{m \in \mathcal{M}} \Delta_{m, n}\right)^{2}} \leq 0 .
\end{aligned}
$$

As a result, $\frac{\partial g_{n}\left(\lambda_{m}, \mu_{n}\right)}{\partial \beta_{m}, n}$ is a monotonically decreasing function with respect to $\beta_{m, n}$ for all $m$. If we have $\left[\frac{\partial g_{n}\left(\lambda_{m}, \mu_{n}\right)}{\partial \beta_{m, n}} \mid \beta_{m, n}=\right.$ $0] \leq 0$, then we may have $\beta_{m, n}^{\star}=0, \forall m$. If we have $\left[\frac{\partial g_{n}\left(\lambda_{m}, \mu_{n}\right)}{\partial \beta_{m}, n} \mid \beta_{m, n}=1\right] \geq 0$, then we may have $\beta_{m, n}^{\star}=1, \forall m$. Otherwise, $\beta_{m, n}^{\star}$ may be derived by solving the following Equation for each network $m$ :

$$
\frac{\Delta_{m, n}^{\prime}}{\sum_{m \in \mathcal{M}} \Delta_{m, n}}+\mu_{n} \Delta_{m, n}^{\prime}-\lambda_{m}=0
$$

which may be solved by the steepest descent method [24]. ${ }^{3}$

At the higher level, we have the master dual problem, which may be expressed as:

$$
\min _{\boldsymbol{\lambda}, \boldsymbol{\mu}} g(\boldsymbol{\lambda}, \boldsymbol{\mu}),
$$

where we have $g(\boldsymbol{\lambda}, \boldsymbol{\mu})=\sum_{m \in \mathcal{M}} \sum_{n \in \mathcal{N}} g_{m, n}\left(\beta_{m, n}^{\star}, \lambda_{m}, \mu_{n}+\right.$ $\sum_{m \in \mathcal{M}} \lambda_{m}-\sum_{n \in \mathcal{N}} \mu_{n} R_{n}$, and $\beta_{m, n}^{\star}$ denotes the optimal value derived from the lower level optimization problem of (35). Since the function $g(\boldsymbol{\lambda}, \boldsymbol{\mu})$ is concave and differentiable, we can use a gradient method for solving the master dual problem, as a benefit of its simplicity. Instead of minimizing the function directly with respect to $\boldsymbol{\lambda}$ and $\boldsymbol{\mu}$, it can be minimized over a single set of Lagrange multipliers first, and then over the remaining one, which may be formulated as minimum $\left.\operatorname{minimum}_{\boldsymbol{\lambda} \geq 0} g(\boldsymbol{\lambda}, \boldsymbol{\mu})\right]$.

Firstly, we solve the minimization problem for a given $\boldsymbol{\mu}$. Then the derivative of $g(\boldsymbol{\lambda}, \boldsymbol{\mu})$ with respect to $\boldsymbol{\lambda}$ is written as:

$$
\frac{\partial g(\boldsymbol{\lambda}, \boldsymbol{\mu})}{\partial \boldsymbol{\lambda}}=1-\sum_{n \in \mathcal{N}} \beta_{m, n}^{\star}
$$

As a result, the price parameter $\boldsymbol{\lambda}$ is updated according to:

$$
\lambda_{m}(t+1)=\left[\lambda_{m}(t)-\xi_{\lambda}(t)\left(1-\sum_{n \in \mathcal{N}} \beta_{m, n}^{\star}\right)\right]^{+},
$$

\footnotetext{
${ }^{3}$ Nonlinear equations can be solved by several numerical methods. However, due to the length limitation of our paper, we will not be able to present the steepest descent method here.
}

TABLE II

Decentralized Algorithm for Problem 1

\begin{tabular}{l}
\hline Algorithm $\mathbf{1}$ \\
\hline Input \\
$\theta_{n}$ : Delay requirement of each MT $n \forall n$, \\
$R_{n}$ : Rate requirement of each MT $n \forall n$ \\
Initialization \\
$t \leftarrow 1$; \\
Price value for each network: $\boldsymbol{\lambda}(t)=\left\{\lambda_{1}(t), \ldots, \lambda_{M}(t)\right\}=\mathbf{0}$ \\
Price value for each MT: $\boldsymbol{\mu}(t)=\left\{\mu_{1}(t), \ldots, \mu_{N}(t)\right\}=\mathbf{0}$ \\
step size: positive $\xi$ and epsilon \\
While $t$ does not reach its maximum \\
$\quad$ Get optimal $\boldsymbol{\beta}$ \\
$\quad$ For each MT $n \in \mathcal{N}$ \\
$\quad$ Each MT solves the problem presented in Eq. (35); \\
$\quad$ End for \\
Update price values \\
$\quad$ For $m \in \mathcal{M}$ \\
$\quad$ Price value $\lambda_{m}$ updates according to Eq. (42) \\
End for \\
$\quad$ For $n \in \mathcal{N}$ \\
$\quad$ Price value $\mu_{n}$ updates according to Eq. (43) \\
End for \\
$\quad \xi \leftarrow \xi t^{-\frac{1}{2}+\epsilon}$ \\
$\quad t \leftarrow t+1$ \\
End \\
\hline Output $\boldsymbol{\beta}^{\star}(t)$ \\
\hline
\end{tabular}

where $[.]^{+}$is a projection on the positive orthant to account for the fact that we have $\lambda_{m} \geq 0$. Furthermore, $\xi_{\lambda}(t)$ denotes the step-size taken in the direction of the negative gradient for the price parameter $\lambda$ at iteration $t$. In order to guarantee convergence, where we have to satisfy $\lim _{t \rightarrow \infty} \xi_{\lambda}(t)=0$ and $\sum_{t=0}^{\infty} \xi_{\lambda}(t)=\infty$. In this paper, we set $\xi_{\lambda}(t)=\xi t^{-\frac{1}{2}+\epsilon}, \xi$ and $\epsilon$ are positive constants.

Then, the price parameter $\boldsymbol{\mu}$ is similarly updated according to:

$$
\mu_{n}(t+1)=\left[\mu_{n}(t)-\xi_{\mu}(t)\left(\sum_{m \in \mathcal{M}} \Delta_{m, n}-R_{n}\right)\right]^{+} .
$$

Based on the above discussions, our decentralized optimal RA scheme is constituted by an iterative algorithm, which determines the optimal transmission probability in the DL of network $m$ to MT $n$ based on the update of a pair of price parameters $\lambda_{m}$ and $\mu_{n}$, over a number of iterations, until the optimal solution is found. Each of the networks $m$ is initialized to a feasible price value $\lambda_{m}$, while each MT $n$ is initialized to a feasible price value $\mu_{n}$. Each MT broadcasts its price value to all the available networks. Then each MT calculates the optimal transmission probability based on the price information $(\boldsymbol{\lambda}, \boldsymbol{\mu})$ and the optimal transmission probability is derived during the last iteration. Each of the networks $m$ updates its price value $\lambda_{m}$ based on the newly derived optimal transmission probability $\beta$ and then broadcasts the optimal transmission probability to the MTs. Similarly, each MT updates its price value $\mu_{n}$ based on the optimal transmission probability. The MTs broadcast their new price values $\boldsymbol{\mu}$ to the networks and the process continues, until the algorithm converges. The decentralized optimal RA algorithm is formally described in Table II. 


\section{B. Decentralized Solution for Multi-Mode MTs}

Problem 2 of (26)-(30) is formulated as a MINLP and may be computationally intractable. A potentially straightforward solution may be to derive firstly the optimal resource block $\boldsymbol{\beta}$ for a specific $\boldsymbol{x}$, then to exhaustive search through the entire set of all the possible $\boldsymbol{x}$ values. For a system having access to $M$ networks and $N$ MTs, there are $M^{N}$ combinations for the network selection indicator variables $\boldsymbol{x}$. Therefore, a simpler solution may be found by relaxing the binary constraint imposed on the network selection indicator variables $x_{m, n}$, so that they may assume continuous values from the interval $[0,1]$. Naturally, the original problem is not actually solved by the relaxation of the binary constraint. However, it has been shown in [39] that solving the dual of the relaxed problem provides solutions that are arbitrarily close to the original, non-relaxed problem.

Since $x_{m, n}$ assumes either the values of 0 or 1 , there is exactly one $x_{m, n}=1$ value for each MT $n$. If we denote such a specific network by the index $m^{\prime}$, we have $\varphi_{\alpha}\left(\sum_{m \in \mathcal{M}}\right.$ $\left.x_{m, n} \Delta_{m, n}\right)=\varphi_{\alpha}\left(\Delta_{m^{\prime}, n}\right)$. Thus, the objective function in (26) is equivalent to the following function:

$$
\max _{\boldsymbol{x}, \boldsymbol{\beta}} \sum_{n \in \mathcal{N}} \sum_{m \in \mathcal{M}} x_{m, n} \varphi_{\alpha}\left(\Delta_{m, n}\right) .
$$

The equivalent relaxed optimization problem of (26)-(30) is reformulated as follows:

$$
\begin{aligned}
\text { Problem 3: } & \max _{\boldsymbol{x}, \boldsymbol{\beta}} \sum_{m \in \mathcal{M}} \sum_{n \in \mathcal{N}} x_{m, n} \varphi_{\alpha}(\Delta m, n) \\
\text { subject to : } & \sum_{m \in \mathcal{M}} x_{m, n} \Delta_{m, n} \geq R_{n}, \forall n \in \mathcal{N}, \\
& \sum_{n \in \mathcal{N}} x_{m, n} \beta_{m, n} \leq 1, \forall m \in \mathcal{M}, \\
& \sum_{m \in \mathcal{M}} x_{m, n}=1, \\
& 0 \leq x_{m, n} \leq 1, \quad 0 \leq \beta_{m, n} \leq 1 .
\end{aligned}
$$

In this formulation, the variables to be optimized are $x_{m, n}$ and $\beta_{m, n}, \forall m, n$.

Lemma 2: The RA problem described by (45)-(49) is a concave optimization problem with respect to the variables $x_{m, n}$ and $\beta_{m, n}$.

Proof: See Appendix B.

Since Problem 3 is also based on a concave function, the optimal solution may be found from the Lagrangian formulation, which may be written as:

$$
\begin{aligned}
& L(\boldsymbol{x}, \boldsymbol{\beta}, \boldsymbol{\lambda}, \boldsymbol{\mu})=\sum_{m \in \mathcal{M}} \sum_{n \in \mathcal{N}}\left[x_{m, n} \varphi_{\alpha}\left(\Delta_{m, n}\right)\right. \\
& \left.-\lambda_{m} \beta_{m, n}+\mu_{n} x_{m, n} \Delta_{m, n}\right]+\sum_{m \in \mathcal{M}} \lambda_{m}-\sum_{n \in \mathcal{N}} \mu_{n} R_{n},
\end{aligned}
$$

where we have $\mathbf{0} \leq \boldsymbol{x} \leq \mathbf{1}, \mathbf{0} \leq \boldsymbol{\beta} \leq \mathbf{1}$ and $\sum_{m \in \mathcal{M}} x_{m, n}=1$. Furthermore, $\mu_{n}$ and $\lambda_{m}$ are the Lagrange multipliers or prices associated with the $n$th inequality constraint of (46) and with the $m$ th inequality constraint of (47), respectively. The optimal RA variables $\boldsymbol{x}, \boldsymbol{\beta}$ may be obtained by solving:

$$
\min _{\boldsymbol{\lambda}, \boldsymbol{\mu}} \max _{\boldsymbol{x}, \boldsymbol{\beta}} L(\boldsymbol{x}, \boldsymbol{\beta}, \boldsymbol{\lambda}, \boldsymbol{\mu}) .
$$

Similarly to Section IV-A, a decentralized optimal RA algorithm can be proposed for solving the problem using full dual decomposition.

We define a dual objective function $g_{n}\left(x_{m, n}, \beta_{m, n}\right)$ for MT $n$, which may be written as:

$$
\begin{aligned}
g_{n}\left(x_{m, n}, \beta_{m, n}\right) & =\sum_{m \in \mathcal{M}} x_{m, n} \varphi_{\alpha}\left(\Delta_{m, n}\right) \\
& -\sum_{m \in \mathcal{M}} \lambda_{m} \beta_{m, n}+\sum_{m \in \mathcal{M}} \mu_{n} x_{m, n} \Delta_{m, n} .
\end{aligned}
$$

At the lower level, we have the subproblems, one for each $n$, which may be written as:

$$
\left\{x_{m, n}^{\star}, \beta_{m, n}^{\star}\right\}=\arg \max _{0 \leq x_{m, n} \leq 1,0 \leq \beta_{m, n} \leq 1} g_{n}\left(x_{m, n}, \beta_{m, n}\right) .
$$

Similarly, $g_{n}\left(x_{m, n}, \beta_{m, n}\right)$ is concave with respect to the variable $x_{m, n}$ and $\beta_{m, n}$. Hence the maximization of $g_{n}\left(x_{m, n}, \beta_{m, n}\right)$ may be derived by finding the partial derivative of $g_{n}\left(x_{m, n}\right.$, $\left.\beta_{m, n}\right)$, which is given by:

$$
\begin{aligned}
\frac{\partial g_{n}}{\partial x_{m, n}} & =\varphi_{\alpha}\left(\Delta_{m, n}\right)+\mu_{n} \Delta_{m, n}-\lambda_{m} \beta_{m, n} \\
\frac{\partial g_{n}}{\partial \beta_{m, n}} & =x_{m, n}\left(\frac{\Delta^{\prime}}{\Delta_{m, n}}+\mu_{n} \Delta_{m, n}^{\prime}-\lambda_{m}\right) .
\end{aligned}
$$

We will firstly derive the optimal RA probability $\beta_{m, n}^{\star}$ according to (55). Since the second partial derivative of $g_{n}\left(x_{m, n}\right.$, $\left.\beta_{m, n}\right)$ with respect to $\beta_{m, n}$ is non-positive, $\beta_{m, n}^{\star}$ is derived according to the following criterion:

1) If $\frac{\Delta_{m, n}^{\prime}(1)}{\Delta_{m, m}(1)}+\mu_{n} \Delta_{m, n}^{\prime}(1)-\lambda_{m} \geq 0$, then $\beta_{m, n}^{\star}=1$;

2) If $\frac{\Delta_{m, n}^{\prime}(0)}{\Delta_{m, m}(0)}+\mu_{n} \Delta_{m, n}^{\prime}(0)-\lambda_{m} \leq 0$, then $\beta_{m, n}^{\star}=0$;

3) Else, the optimal RA probability $\beta_{m, n}^{\star}$ from network $m$ and MT $n$ is derived by solving the equation $\frac{\Delta_{m, n}^{\prime}\left(\beta_{m, n}^{\star}\right)}{\Delta_{m, m}\left(\beta_{m, n}^{\star}\right)}+\mu_{n} \Delta_{m, n}^{\prime}\left(\beta_{m, n}^{\star}\right)-\lambda_{m}=0$.

The optimal network selection index $x_{m, n}^{\star}$ is then determined according to the $\beta_{m, n}^{\star}$ derived. Each MT $n$ calculates the partial derivative of $g_{n}\left(x_{m, n}, \beta_{m, n}^{\star}\right)$ in the direction of $x_{m, n}$ for all the networks. Then MT $n$ chooses the specific network $m^{\prime}$ associated with the highest value, which may be written as:

$$
\begin{aligned}
& x_{m^{\prime}, n}^{\star}=1, \text { if } m^{\prime}=\arg \max _{\forall m} \frac{\partial g_{n}\left(x_{m, n}, \beta_{m, n}^{\star}\right)}{\partial x_{m, n}} ; \\
& x_{m, n}^{\star}=0, \text { if } m \neq m^{\prime} .
\end{aligned}
$$

At the higher level, we have the master dual problem, which may be expressed as:

$$
\min _{\boldsymbol{\lambda}, \boldsymbol{\mu}} g(\boldsymbol{\lambda}, \boldsymbol{\mu})
$$


TABLE III

Decentralized Algorithm for Problem 2

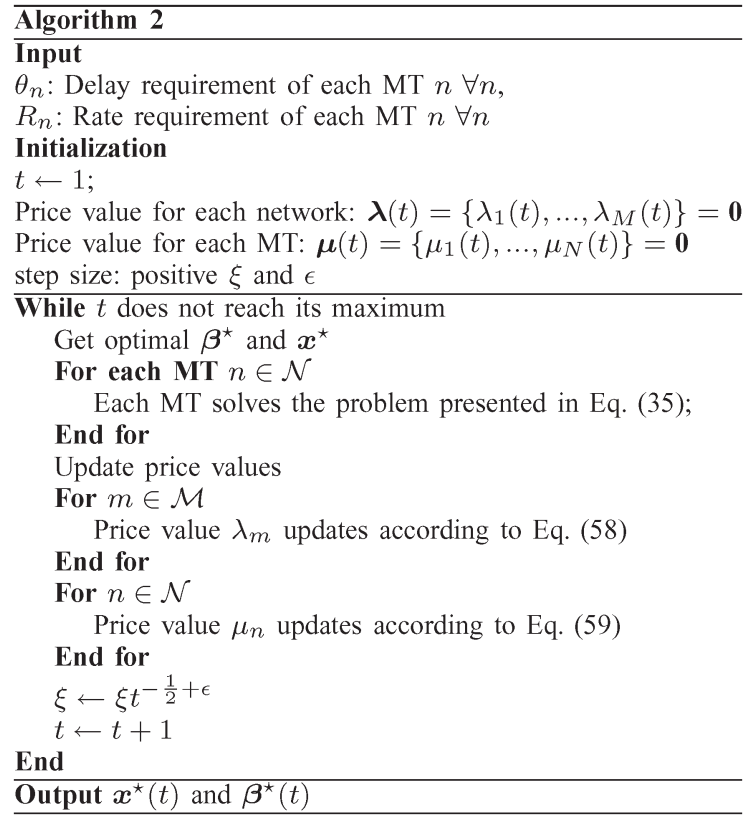

where we have $g(\boldsymbol{\lambda}, \boldsymbol{\mu})=\sum_{n \in \mathcal{N}} g_{n}\left(x_{m, n}^{\star}, \beta_{m, n}^{\star}\right)+\sum_{m \in \mathcal{M}} \lambda_{m}-$ $\sum_{n \in \mathcal{N}} \mu_{n} R_{n}$, and $x_{m, n}^{\star}, \beta_{m, n}^{\star}$ denotes the optimal value derived from the lower level optimization problem (53). Similar to the solution provided in Section IV-A, the price parameters $\lambda$ and $\boldsymbol{\mu}$ are updated according to:

$$
\begin{aligned}
\lambda_{m}(t+1) & =\left[\lambda_{m}(t)-\xi_{\lambda}(t)\left(1-\sum_{n \in \mathcal{N}} \beta_{m, n}^{\star}\right)\right]^{+} \\
\mu_{n}(t+1) & =\left[\mu_{n}(t)-\xi_{\mu}(t)\left(x_{m, n}^{\star} \sum_{n \in \mathcal{N}} \Delta_{m, n}\left(\beta_{m, n}^{\star}\right)-R_{n}\right)\right]^{+} .
\end{aligned}
$$

Similarly, our decentralized optimal RA algorithm is an iterative algorithm, which performs an optimal network selection and finds the corresponding resource block probability from network $m$ to MT $n$ based on the update of the price parameters $\lambda_{m}$ and $\mu_{n}$, over a number of iterations, until the optimal solution is reached. Each of the networks $m$ starts with an feasible initial price value $\lambda_{m}$, while each MT $n$ starts with an feasible initial price value $\mu_{n}$. Each MT broadcasts its price value to all networks. Then each network calculates the optimal network selection $x_{m, n}^{\star}$ and transmission resource block probability $\beta_{m, n}^{\star}$ based on the price information $(\boldsymbol{\lambda}, \boldsymbol{\mu})$. Each network $m$ updates its price value $\lambda_{m}$ and then broadcasts the optimal transmission resource block to the MTs. Similarly, each MT updates its price values $\mu_{n}$. The MTs broadcast their new price values $\boldsymbol{\mu}$ to the networks and the process continues, until the algorithm converges. The decentralized optimal RA algorithm is formally described in Table III.
TABLE IV

\begin{tabular}{|c|c|}
\hline \multicolumn{2}{|l|}{ 2D indoor scenario } \\
\hline Length of room & $20 \mathrm{~m}$ \\
\hline Height of room & $3 \mathrm{~m}$ \\
\hline Number of indoor MT & 10 \\
\hline Height of MT & $0.85 \mathrm{~m}$ \\
\hline Number of rate guaranteed MT & 4 \\
\hline BER target of MTs & $10^{-5}$ \\
\hline Guaranteed effective capacity & 10/20 (Mbit/s) \\
\hline Number of iteration & 100 \\
\hline \multicolumn{2}{|l|}{ VLC System } \\
\hline Height of LED & $2.5[\mathrm{~m}]$ \\
\hline Power of LED & $20[\mathrm{w}]$ \\
\hline Semi-angle at half power & 70 [deg.] \\
\hline Width of the field of view & 60 [deg.] \\
\hline Detector physical area of a PD & $1.0\left[\mathrm{~cm}^{2}\right]$ \\
\hline Refractive index of a lens at a PD & 1.5 \\
\hline $\mathrm{O} / \mathrm{E}$ conversion efficiency & $0.53[\mathrm{~A} / \mathrm{W}]$ \\
\hline Available bandwidth for VLC system & $10[\mathrm{MHz}]$ \\
\hline Reflectance factor & 0.8 \\
\hline Electronic charge & $1.6 \times 10^{-19}[\mathrm{C}]$ \\
\hline Background current & $5.1 \times 10^{-3}[\mathrm{~A}]$ \\
\hline Open-loop voltage gain & 10 \\
\hline Fixed capacitance of the PD per unit area & $1.12 \times 10^{-6}$ \\
\hline FET channel noise factor & 1.5 \\
\hline FET transconductance & $3 \times 10^{-2}$ \\
\hline \multicolumn{2}{|l|}{ Femtocell System } \\
\hline Position of the indoor femtocell & $(0,0)$ \\
\hline Transmission power of the femtocell BS & $0.02[\mathrm{w}]$ \\
\hline Indoor path-loss exponent & $3>$ \\
\hline Carrier frequency & $2[\mathrm{GHz}]$ \\
\hline Indoor path-loss constant & $37[\mathrm{~dB}]$ \\
\hline Available bandwidth for femtocell system & $5[\mathrm{MHz}]$ \\
\hline
\end{tabular}

Notations AND SySTEM PARAMETERS

\section{RESUlTS AND Discussions}

In this section, we present numerical performance results for characterizing the proposed RA algorithms in the context of the indoor HetNet of Fig. 1. Again, the "two-rate" transmission channel model is used for the VLC system, while the transmission channel is subject to uncorrelated Rayleigh fading with a unity average power and to propagation loss for the RF femtocell system. We assume that 10 MTs are uniformly distributed in the room and all experience the same delay exponents. Furthermore, the MTs are divided into two groups, where four guaranteed-rate MTs are in one of the groups, while no guaranteed rate is maintained for the MTs in the other group. Our main system parameters are summarized in Table IV.

\section{A. Convergence of the Distributed Iterative Algorithm}

Fig. 2 illustrates the convergence behavior of our distributed RA algorithm for both sophisticated multi-homing MTs and for the less advanced multi-mode MTs at a zero VLC blocking probability of $p=0$, in conjunction with $\theta=0.01$ and $\alpha=1$. For comparison, we use the BONMIN solver [37] in order to derive the optimal solution of our RA problem. As shown in Fig. 2, our distributed RA algorithm converges to the optimal value within 100 iterations for both the multi-homing MTs and for the multi-mode MTs. This result demonstrates that the distributed RA algorithm indeed finds the optimal RA probability for multi-homing MTs as well as the optimal RA probability and network selection for the multi-mode MTs, respectively. Observe furthermore from Fig. 2 that the objective function value for multi-homing MTs is higher than that for the 


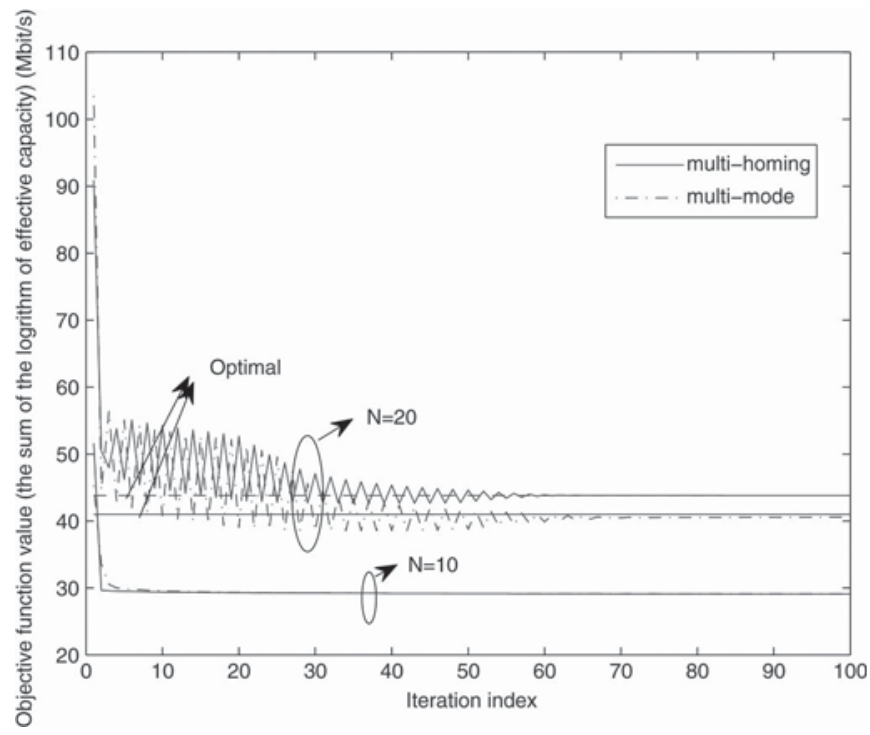

Fig. 2. Objective function (OF) values versus the total number of iterations for the proposed decentralized algorithms for both multi-homing MTs and multimode MTs, in conjunction with $\alpha=1, \theta=0.01$, and $p=0$.

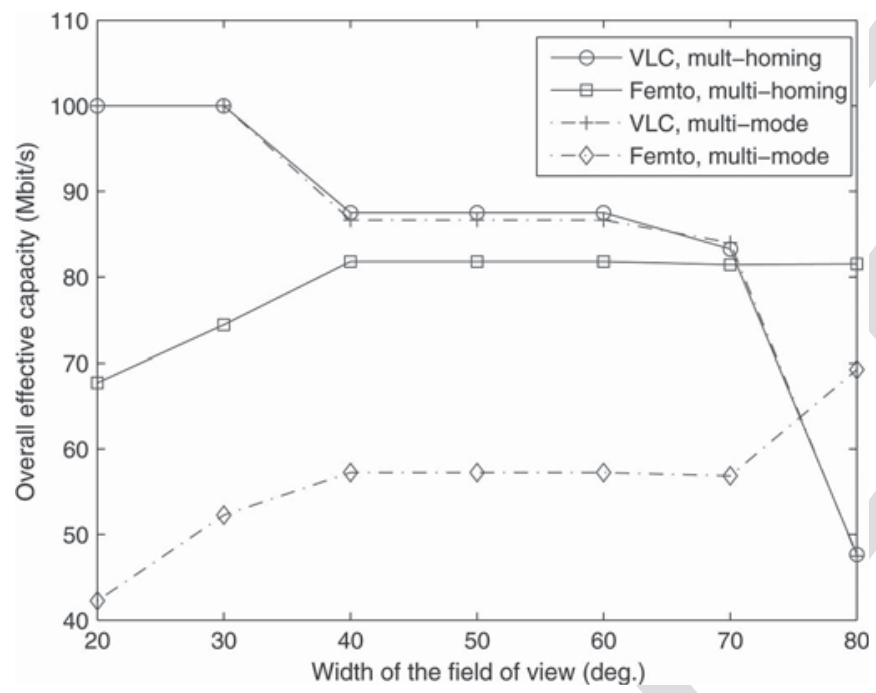

Fig. 3. The overall effective capacity of MTs versus the field of view of MTs, for $\alpha=1, \theta=1$, and $p=0$.

multi-mode MTs. This is because the RA problem of the multimode MTs might be viewed as a specific scenario of the multihoming MTs. We may also note that the speed of convergence is slower, when we increase the number of MTs.

\section{B. Effect of the Width Field of View Angle and of the Semi-Angle at Half Power}

Fig. 3 demonstrates the relationship between the FOV and the overall effective capacity of the MTs for the delay exponent of 1 and for the VLC LOS blocking probability of $p=0$. Observe from the figure that the VLC system's effective capacity is non-increasing, when we increase the angle of the FOV. This is because the gain of the optical concentrator decreases upon the increasing the FOV. We also note that the overall effective capacity of the VLC system is substantially reduced, when the FOV is larger than $70^{\circ}$. Hence in our simulation scenario the FOV of MTs should be no larger than $70^{\circ}$. Furthermore,

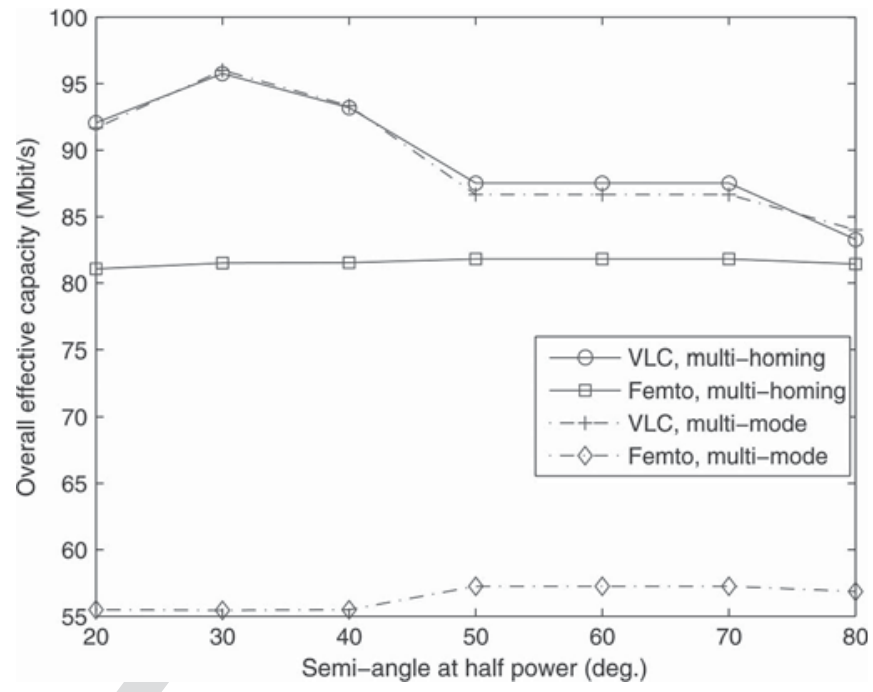

Fig. 4. The overall effective capacity of MTs versus the semi-angle at half power of the lights, for $\alpha=1, \theta=1$, and $p=0$.

it is also shown that the overall effective capacity of the RF femtocell system increases slightly. The reason might be that the specific MTs that suffer from the performance degradation of the VLC system may be compensated by having a good channel quality for the RF femtocell system.

Fig. 4 shows the overall effective capacity of the MTs as a function of the semi-angle $\phi_{1 / 2}$ at half power of the lights, for the delay exponent of 1 and VLC LOS blocking probability of $p=0$. It is observed that the VLC system's effective capacity increases, when $\phi_{1 / 2}$ increases from $20^{\circ}$ to $30^{\circ}$. Then the effective capacity decreases, when $\phi_{1 / 2}$ increases from $30^{\circ}$ to $80^{\circ}$. This is because the value of the semi-angle at half power affects the order of Lambertian emission. As a result, the direct LOS ray and the first reflected DC gain of (1) and (5) may change according to the value of $\phi_{1 / 2}$.

\section{Effective Capacity of MTs: Multi-Homing Versus Multi-Mode MTs}

Let us now compare the performance of multi-homing MTs and multi-mode MTs for different values of $\alpha$. It is observed that the performance of the multi-homing MTs is better than that of the multi-mode MTs. Again, this is because the multimode transmission may be viewed as a specific scenario of multi-homing. Hence, the performance of multi-mode MTs may be interpreted as the lower bound of the maximum achievable capacity of multi-homing MTs. When we set $\alpha=0$, as shown in Fig. 5, the RA problem is reformulated as the maximization of the total effective capacity. Since user 5 and user 6 are located in the middle of the room and are capable of achieving a better performance for the RF femtocell, the femtocell system is willing to allocate more resources to these two MTs in the multi-homing scenario.

Furthermore, owing to the rate constraint, the guaranteedrate MTs always satisfy the minimum rate requirement. For the multi-homing scenario, the VLC system and the RF femtocell system may simultaneously transmit to MT 4 in order to satisfy the rate requirement. However, for the multi-mode scenario, the femtocell system may allocate the resource to MT 4 in order 

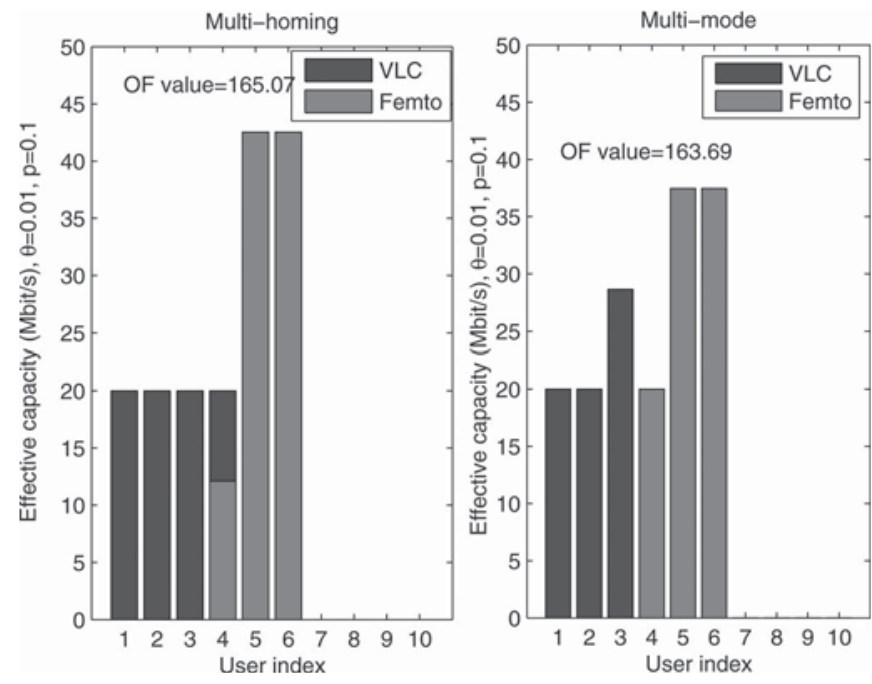

Fig. 5. The effective capacity of both multi-homing MTs and multi-mode MTs, along with $\alpha=0, \theta=0.01$, and $p=0.1$.
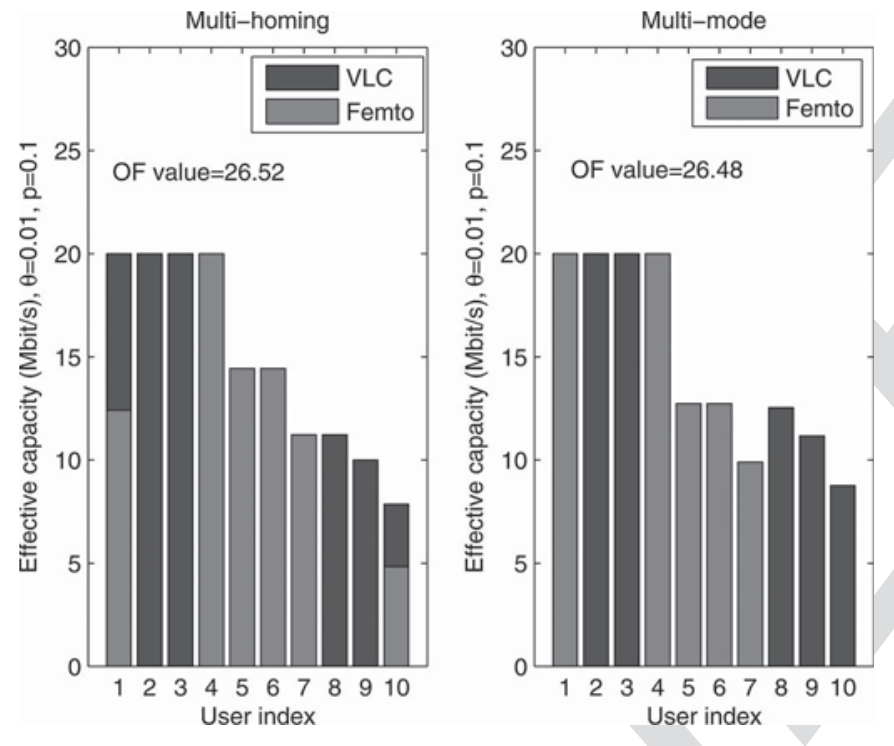

Fig. 6. The effective capacity of both multi-homing MTs and multi-mode MTs, with $\alpha=1, \theta=0.01$, and $p=0.1$.

to satisfy its rate requirement This is because the VLC system may not be able to simultaneously satisfy all of the four MTs' rate requirement. For the non-rate-guaranteed MTs, no resource will be allocated, when $\alpha=0$. This is because the VLC system prefers to allocate resources to the guaranteed-rate MTs, and the femtocell system may allocate resources to the centrally located MTs, in order to achieve the maximum total effective capacity.

When we set $\alpha=1$, observe in Fig. 6 that the RA problems are formulated under the proportional fairness constraint. It is illustrated that the objective function value for MT-homing MTs is higher than that for MT-mode MTs. The rate guaranteed MTs have higher priority and their rate requirements are satisfied for both the multi-homing and the multi-mode scenarios. It may be observed in Fig. 6 that the MTs located in the middle of the room may achieve a higher effective capacity for multihoming MTs. This is because the MTs in the middle of the room are capable of achieving a better performance, when communicating with the RF femtocell system. In contrast to
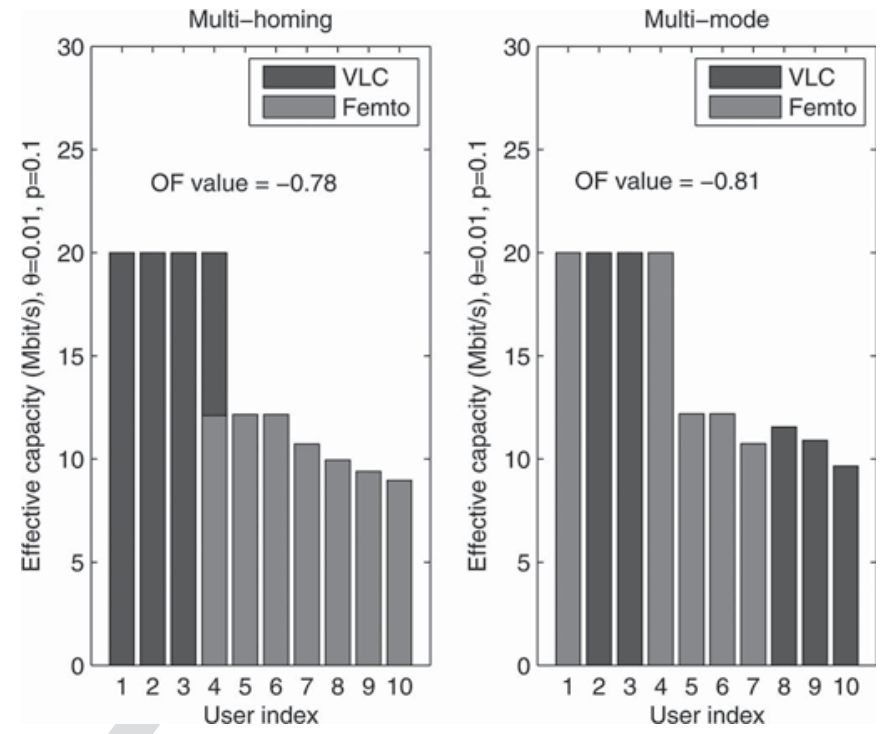

Fig. 7. The effective capacity of both multi-homing MTs and multi-mode MTs, with $\alpha=2, \theta=0.01$, and $p=0.1$.

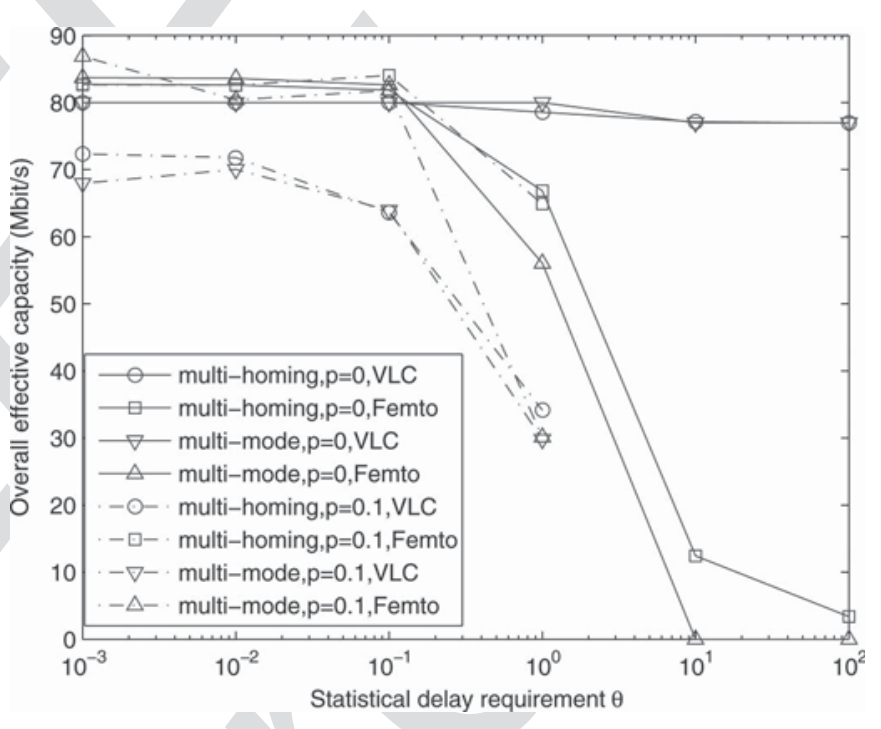

Fig. 8. The overall effective capacity of MTs versus the statistical delay parameter $\theta$, for $\alpha=1$.

the scenario of $\alpha=0$, the system may allocate resources to the non-guaranteed-rate MTs under the consideration of fairness to all MTs. Hence MT 7, 8, 9, 10 also receive their signals from the VLC system or from the femtocell system. When the MTs are of the multi-mode type, the center MTs may choose the RF femtocell system for their transmission, while the VLC system may transmit to the edge MTs. Furthermore, observe in Fig. 7 that when we increase the value of $\alpha$, the differences of effective capacity upon receiving from a specific network between the different MTs are smaller than that when we set $\alpha=1$. As a result, we believe that having a higher $\alpha$ results in a higher grade of fairness.

\section{Effect of the Delay Statistics}

Fig. 8 illustrates the effect of the delay exponent $\theta$ on the overall effective capacity of the MTs for the blocking 
probabilities of $p=0$ and $p=0.1$, respectively. Observe from the figure that the overall effective capacity is reduced upon increasing the delay exponent $\theta$. However, the overall effective capacity of both the VLC and of the RF femtocell system is fairly insensitive to the delay exponent, when the exponent is relatively small. This is because when the delay exponent is low, the resultant delay requirement is loose and the overall effective capacity is close to the Shannon capacity, which depends on the wireless channel, but is independent of the delay of the packetarrival process. However, the overall effective capacity of the RF femtocell decreases substantially, when the delay exponent is increased. Furthermore, when the delay exponent is relatively high, the overall effective capacity tends to zero. However, it is shown in Fig. 8 that the overall effective capacity of the VLC remains almost unchanged for the entire delay exponent region considered for $p=0$. The DL effective capacity between the VLC LEDs and MT $n$ is expressed as: $\Delta_{1, n}\left(\beta_{1, n}\right)=\beta_{1, n} \mathcal{R}_{1, n}$, which is independent of the delay exponent $\theta$. Observe from Fig. 8 that the effective capacity gleaned from the VLC system decreases only slightly, while the effective capacity of the RF femtocell decreases rapidly. This is because the VLC system increases the amount of resources allocated to the guaranteedthroughput MTs, at the cost of decreasing the overall effective capacity. Hence, the VLC system benefiting from a zero blocking probability is more reliable than the RF femtocell link, when the MTs have to satisfy a certain delay constraint.

When we assume that the blocking probability of the VLC system equals to 0.1 , its performance degrades rapidly for $\theta \geq 0.1$. Hence naturally, the VLC system is more sensitive to the delay constraints in the presence of a non-zero blocking probability. As a result, the RF femtocell has to increase its RA probability, especially to the MTs operating under a strict bit rate guarantee. When we have $\theta>1$, it is observed in Fig. 8 that our system fails to fulfil the bit rate requirement. In this case, no optimal solution can be found. It is also observed in Fig. 8 that the attainable performance is similar for multi-homing MTs and multi-mode MTs, when the delay requirement is loose.

\section{E. Effect of the VLC System's Blocking Probability}

Let us now quantify the effect of the VLC system's blocking probability on the overall effective capacity of the MTs, when using a delay exponent of 1. It is illustrated in Fig. 9 that the VLC system's effective capacity decreases, when the blocking probability is increased. However, as seen in Fig. 9, the effective capacity of the RF femtocell system is slightly reduced for the multi-homing MTs. The RF femtocell system should increase the amount of resources allocated to the MTs, if they have to satisfy a certain guaranteed throughput, which is achieved at the price of decreasing the overall effective capacity. When the MTs are multi-mode terminals, we observe that the overall effective capacity of the VLC system and RF femtocell system substantially decreases at $p=0.1$. This is because when the VLC LOS reception is blocked with a certain probability, the effective capacity is substantially reduced according to (18) and the first reflected ray may only contribute to the MTs located near the wall. As a result, the RF femtocell system may have to allocate resources to the guaranteed-rate MTs,

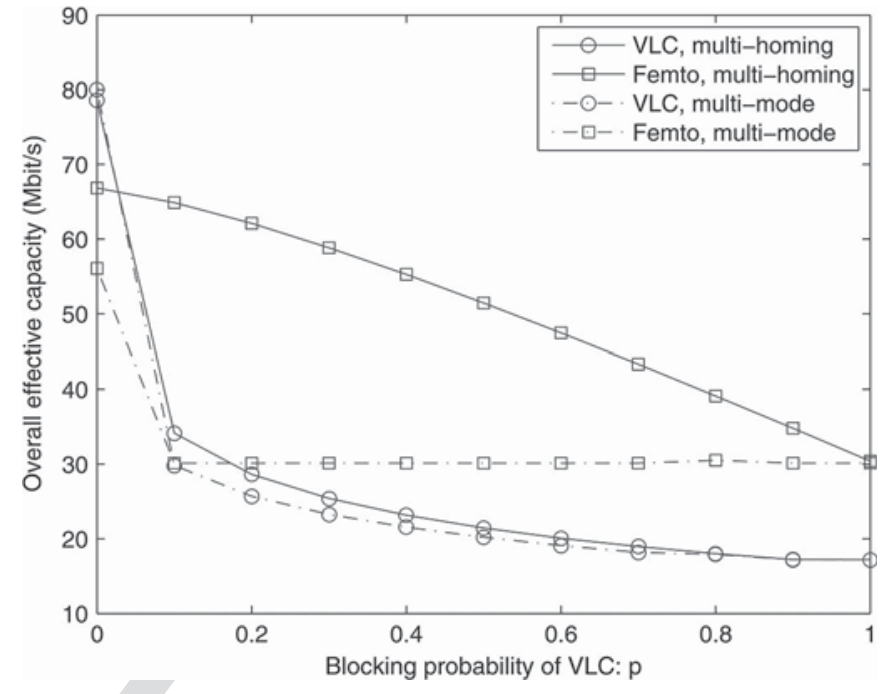

Fig. 9. The overall effective capacity of MTs versus the blocking probability of the VLC system $p$, for $\alpha=1$ and $\theta=1$.

even if their RF channel quality is not particularly good. When the LOS reception blocking probability increases from $p=0.1$ to $p=1$, the overall effective capacity of the RF femtocell system remains constant. This is because the femtocell system may allocate most of its resources to the guaranteed-rate MTs. Increasing $p$ results in decreasing the performance of the VLC system. As a result, the overall effective capacity of the VLC system keeps on decreasing. When $p=1$, the LOS ray is blocked, hence the MTs may only receive a reduced optical power due to the first reflected ray. Hence, it is plausible that when the blocking probability of the VLC system is high, the $\mathrm{RF}$ femtocell system becomes more reliable.

\section{F. Effect of the User Distribution}

In the above simulations, we assume that the MTs are uniformly located in the room. Fig. 10 compares the overall effective capacity, when all the MTs are located in the center (center cluster) or at the edge (edge cluster). Observe that the performance of the RF femtocell system of center-cluster MTs is better than that of the edge-cluster MTs. This is because we assume that the RF femtocell BS is positioned in the center of the room. As a result, the center-cluster MTs benefit from a shorter transmission distance and a lower pathloss. We also observe that the overall effective capacity of the VLC system decreases upon increasing the VLC LOS reception blocking probability $p$. When the MTs are center-clustered, the effective capacity of the VLC system is substantially reduced, as $p$ increases from 0 to 0.1 . Then it starts to decay toward 0 for larger $p$ values. The performance of the VLC system is sensitive to the LOS reception blocking, especially when the MTs are located in the center of the room. However, when the MTs are edge-clustered, the VLC system may still be capable of supporting an approximately $30 \mathrm{Mbit} / \mathrm{s}$ transmission rate, even if the VLC LOS blocking probability becomes 1 , because the edge MTs may still benefit from the reflected optical power. 

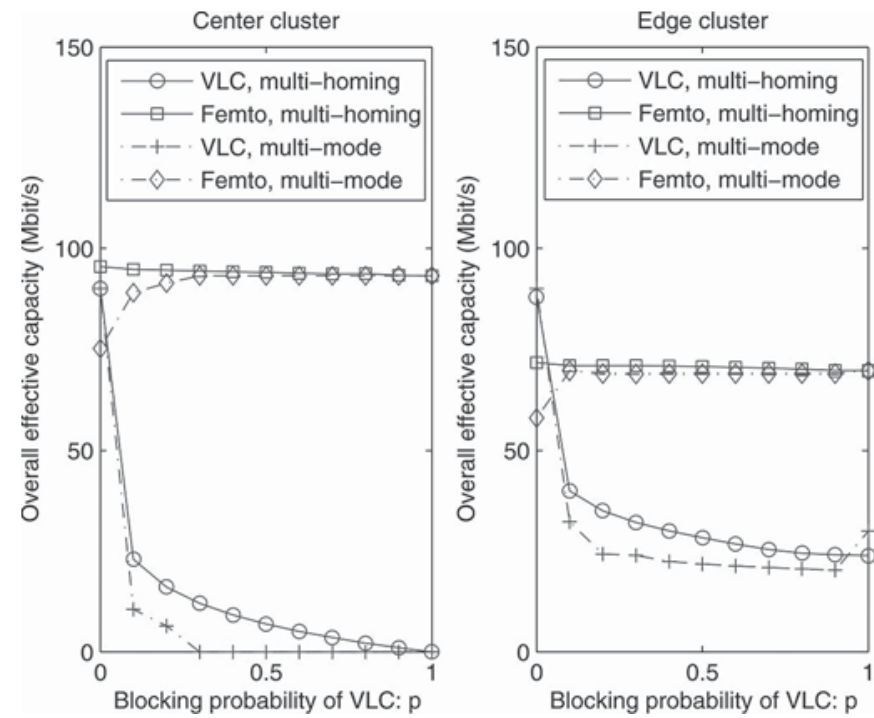

Fig. 10. The overall effective capacity of MTs versus the blocking probability of $p$, when the MTs are located at the room-center and room-edge, for $\alpha=1$ and $\theta=1$.

\section{CONCLUSION}

In this paper, we studied the resource allocation problems of mobile terminals (MTs) in a HetNet under diverse QoS requirements in terms of the data rate, fairness to MTs and the statistical delay requirements, where a hybrid VLC and RF femtocell system was considered. The objective functions relied upon were proven to be concave. Then we proposed decentralized algorithms for solving the associated RA problem. The optimal RA for each iteration of the dual decomposition algorithm were presented and simulations were performed for validating the algorithm.

Our simulation results compared the performance of the multi-homing MTs and of the multi-mode MTs in conjunction with different values of $\alpha$. Multi-homing MTs are capable of achieving a better performance than multi-mode MTs. Furthermore, our simulation results illustrated that when the VLC LOS blocking probability is zero, the VLC system is more reliable than the RF femtocell link, even if the statistical delay constraints are tight. However, it is plausible that when the VLC LOS blocking probability is high, the RF femtocell system becomes more reliable. Additionally, a comprehensive study of the effects of various system parameters was carried out.

\section{APPENDIX A}

\section{PROOF OF LEMMA 1}

Firstly, we prove that the effective capacity $\Delta_{m, n}\left(\beta_{m, n}\right)$ of the link spanning from network $m$ to MT $n$ is a concave function of $\beta_{m, n}$. The first derivative of the effective capacity $\Delta_{1, n}\left(\beta_{1, n}\right)$ from the VLC link to MT $n$ is given by:

$$
\frac{d \Delta_{1, n}}{d \beta_{1, n}}=\frac{\mathcal{R}_{2, n} p e^{-\theta_{n} \mathcal{R}_{2, n} \beta_{1, n}}+\mathcal{R}_{1, n}(1-p) e^{-\theta_{n} \mathcal{R}_{1, n} \beta_{1, n}}}{p e^{-\theta_{n} \mathcal{R}_{2, n} \beta_{1, n}}+(1-p) e^{-\theta_{n} \mathcal{R}_{n} \beta_{1, n}}} .
$$

Then the second derivative of the effective capacity $\Delta_{1, n}$ from the VLC link to MT $n$ is given by:

$$
\begin{aligned}
\frac{d^{2} \Delta_{1, n}}{d \beta_{1, n}^{2}} & =-\frac{\left(\mathcal{R}_{1, n}-\mathcal{R}_{2, n}\right)^{2} \theta_{n} p(1-p) e^{-\theta_{n} \beta_{1, n}\left(\mathcal{R}_{1, n}+\mathcal{R}_{2, n}\right)}}{\left[p e^{-\theta_{n} \mathcal{R}_{2, n} \beta_{1, n}}+(1-p) e^{-\theta_{n} \mathcal{R}_{1, n} \beta_{1, n}}\right]^{2}} \\
& \leq 0, \text { for } \theta_{n} \geq 0,0 \leq p \leq 1 .
\end{aligned}
$$

The second derivative of the effective capacity $\Delta_{2, n}$ from the RF femtocell link to MT $n$ is given by (62), shown at the bottom of the page, where inequality $a$ holds, according to the Cauchy-Schwarz inequality property presented in [40].

Assuming that we have $f_{1}\left(\beta_{m, n}\right)=\varphi_{\alpha}\left(\sum_{m \in \mathcal{M}} \Delta_{m, n}\right)$, the second partial derivative of $f_{1}\left(\beta_{m, n}\right)$ may be written as:

$$
\begin{aligned}
\frac{\partial^{2} f_{1}\left(\beta_{m, n}\right)}{\partial \beta_{m, n}^{2}} & =\frac{\Delta_{m, n}^{\prime \prime} \sum_{m \in \mathcal{M}} \Delta_{m, n}\left(\beta_{m, n}\right)-\left(\Delta_{m, n}\right)^{2}}{\left(\sum_{m \in \mathcal{M}} \Delta_{m, n}\right)^{2}} \\
& \leq 0 .
\end{aligned}
$$

As a result, the objective function (22) of Problem 1 is a concave function with respect to $\beta_{m, n}$. The linear transformations of a concave function still constitute a function, hence the problem described by (22)-(25) is a concave problem.

\section{APPENDIX B}

\section{PROOF OF LEMMA 2}

Firstly, we assume that $f_{2}\left(x_{m, n}, \beta_{m, n}\right)=x_{m, n} \varphi_{\alpha}\left(\Delta_{m, n}\right)$. Let us now use the Hessian matrix for examining the concavity of the function $f_{2}\left(x_{m, n}, \beta_{m, n}\right)$, which is given by:

$$
\mathcal{H}\left(f_{2}\right)=\left[\begin{array}{cc}
\frac{\partial^{2} f_{2}}{\partial \beta_{2}^{2}} & \frac{\partial^{2} f_{2}}{\partial \beta_{m, n} \partial x_{m}, n} \\
\frac{\partial^{2} f_{2}}{\partial x_{m}, n \partial \beta_{m, n}} & \frac{\partial^{2} f_{2}}{\partial x_{m, n}^{2}},
\end{array}\right],
$$

$$
\begin{aligned}
\frac{d^{2} \Delta_{2, n}\left(\beta_{2, n}\right)}{d \beta_{2, n}^{2}}=\frac{\theta_{n} B_{f}^{2}}{\ln ^{2} 2}\left\{\frac{\left[\int_{0}^{\infty}(1+x)^{\frac{-\theta_{n} B_{f} \beta_{2, n}}{\ln 2}} e^{\frac{-x}{\Gamma}} \ln (1+x) d x\right]^{2}}{\left[\int_{0}^{\infty}(1+x)^{\frac{-\theta_{n} B_{f} \beta_{2, n}}{\ln 2}} e^{\frac{-x}{\Gamma}} d x\right]^{2}}\right. & \\
& \left.-\frac{\int_{0}^{\infty}(1+x)^{\frac{-\theta_{n} B_{f} \beta_{2, n}}{\ln 2}} e^{\frac{-x}{\Gamma}} \ln ^{2}(1+x) d x \int_{0}^{\infty}(1+x)^{\frac{-\theta_{n} B_{f} \beta_{2, n}}{\ln 2}} e^{\frac{-x}{\Gamma}} d x}{\left[\int_{0}^{\infty}(1+x)^{\frac{-\theta_{n} B_{f} \beta_{2, n}}{\ln 2}} e^{\frac{-x}{\Gamma}} d x\right]^{2}}\right\} \\
& \leq 0
\end{aligned}
$$


where $\frac{\partial^{2} f_{1}}{\partial \beta_{m, n}^{2}}$ and $\frac{\partial^{2} f_{1}}{\partial x_{m, n}^{2}}$ are the principle minors of the Hessian matrix. Recalling the proof of Lemma 1 in Appendix A, it may be readily seen that the effective capacity of each MT $\Delta_{m, n}$ is concave functions with respect to $\beta_{m, n}$. As a result, $\frac{\partial^{2} f_{2}}{\partial \beta_{m, n}^{2}}=$ $\frac{x_{m, n} \Delta_{m, n}^{\prime \prime}-x_{m, n}\left(\Delta_{m, n}^{\prime}\right)^{2}}{\left(\Delta_{m, n}\right)^{2}}$ is non-positive. Furthermore, we have $\frac{\partial^{2} f_{1}}{\partial x_{m, n}^{2}}=0$. Hence all the principle minors of the Hessian matrix are non-positive, and therefore the function $f_{2}\left(x_{m, n}, \beta_{m, n}\right)$ is concave with respect to the variables $x_{m, n}$ and $\beta_{m, n}$.

Similarly, we can readily show that the constraint described by (46) is concave with respect to the variables $x_{m, n}$ and $\beta_{m, n}$. The constraints described by (47)-(49) are linear. As a result, Problem 3 is a concave optimization problem.

\section{REFERENCES}

[1] R. Zhang and L. Hanzo, "Wireless cellular networks," IEEE Veh. Technol. Mag., vol. 5, no. 4, pp. 31-39, Dec. 2010.

[2] L. Hanzo et al., "Wireless myths, realities, futures: From 3G/4G to optical and quantum wireless," Proc. IEEE, vol. 100, pp. 1853-1888, May 2012.

[3] D. Tsonev, S. Videv, and H. Haas, "Light fidelity (Li-Fi): Towards alloptical networking," in Proc. SPIE, Feb. 2013, pp. 900 702-1-900 702-10.

[4] S. Brueck, "Heterogeneous networks in LTE-Advanced," in Proc. 8th Int. Symp. Wireless Commun. Syst., Aachen, Germany, Nov. 2011, pp. 171-175.

[5] F. Jin, R. Zhang, and L. Hanzo, "Frequency-swapping aided femtocells in twin-layer cellular networks relying on fractional frequency reuse," in Proc. IEEE WNCN, Paris, France, Apr. 2012, pp. 3097-3101.

[6] F. Jin, R. Zhang, and L. Hanzo, "Fractional frequency reuse aided twin-layer femtocell networks: Analysis, design and optimization," IEEE Trans. Commun., vol. 61, no. 5, pp. 2074-2085, May 2013.

[7] C. L. Tsai and Z. Xu, "Line-of-sight visible light communications with InGaN-based resonant cavity LEDs," IEEE Photon. Technol. Lett., vol. 25, no. 18, pp. 1793-1796, Sep. 2013.

[8] I. Stefan, H. Burchardt, and H. Haas, "Area spectral efficiency performance comparison between VLC and RF femtocell networks," in Proc. IEEE Int. Conf. Commun., Budapest, Hungary, Jun. 2013, pp. 3825-3829.

[9] C. Luo, H. Ji, and Y. Li, "Utility based multi-service bandwidth allocation in the 4G heterogeneous wireless access networks," in Proc. IEEE WNCN, Budapest, Hungary, Apr. 2009, pp. 1-5.

[10] M. Ismail and W. Zhuang, "A distributed multi-service resource allocation algorithm in heterogeneous wireless access medium," IEEE J. Sel. Areas Commun., vol. 30, no. 2, pp. 425-432, Feb. 2012.

[11] M. Ismail and W. Zhuang, "Decentralized radio resource allocation for single-network and multi-homing services in cooperative heterogeneous wireless access medium," IEEE Trans. Wireless Commun., vol. 11, no. 11, pp. 4085-4095, Nov. 2012.

[12] M. Ismail, A. Abdrabou, and W. Zhuang, "Cooperative decentralized resource allocation in heterogeneous wireless access medium," IEEE Trans. Wireless Commun., vol. 12, no. 2, pp. 714-724, Feb. 2013.

[13] D. Wu and R. Neigi, "Effective capacity: A wireless link model for support of quality of service," IEEE Trans. Wireless Commun., vol. 2, no. 4, pp. 630-643, Jul. 2003.

[14] M. Tao, Y. Liang, and F. Zhang, "Resource allocation for delay differentiated traffic in multiuser OFDM systems," IEEE Trans. Wireless Commun., vol. 7, no. 6, pp. 2190-2201, Jun. 2008.

[15] D. S. W. Hui, V. K. N. Lau, and H. L. Wong, "Cross-layer design for OFDMA wireless systems with heterogeneous delay requirements," IEEE Trans. Wireless Commun., no. 8, pp. 2872-2880, Aug. 2007.

[16] J. Tang and X. Zhang, "Cross-layer resource allocation over wireless relay networks for quality of service provisioning," IEEE J. Sel. Areas Commun., vol. 25, no. 4, pp. 2318-2328, May 2007.

[17] Y. Cui, V. K. N. Lau, R. Wang, H. Huang, and S. Zhang, "A survey on delay-aware resource control for wireless systems-Large deviation theory, stochastic Lyapunov, distributed stochastic learning," IEEE Trans. Inf. Theory, vol. 58, no. 3, pp. 1677-1701, Mar. 2012.

[18] N. McKeown, A. Mekkittikul, V. Anantharam, and J. Walrand, "Achieving 100 percent throughput in an input-queued switch," IEEE Trans. Commun., vol. 47, no. 8, pp. 1260-1267, Aug. 1999.
[19] L. Tassiulasa and A. Ephremides, "Dynamic sever allocation to parallel queues with randomly varying connectivity," IEEE Trans. Inf. Theory, vol. 39, no. 2, pp. 466-478, Mar. 1993.

[20] M. Uchida and J. Kurose, "An information-theoretic characterization of weighted $\alpha$-proportional fairness," in Proc. IEEE INFOCOM, Rio de Janeiro, Brazil, Apr. 2009, pp. 1053-1061.

[21] J. Mo and J. Walrand, "Fair end-to-end window-based congestion control," IEEE/ACM Trans. Netw., vol. 8, no. 5, pp. 556-567, Oct. 2000.

[22] Y. Yi and M. Chiang, "Stochastic network utility maximization: A tribute to Kelly's paper published in this journal a decade ago," Eur. Trans. Telecommun., vol. 19, no. 4, pp. 421-442, Jun. 2008.

[23] A. Sang, X. Wang, M. Madihian, and R. D. Gitlin, "A flexible downlink scheduling scheme in cellular packet data systems," IEEE Trans. Wireless Commun., vol. 5, no. 3, pp. 568-577, Mar. 2006.

[24] S. Boyd and L. Vandenberghe, Convex Optimization. Cambridge, U.K.: Cambridge Univ. Press, 2004.

[25] M. Chiang, S. H. Low, A. R. Calderbank, and J. Doyle, "Layering as optimization decomposition: A mathematical theory of network architectures," Proc. IEEE, vol. 95, no. 1, pp. 255-312, Jan. 2007.

[26] F. R. Gfeller and U. Bapst, "Wireless in-house data communication via diffuse infrared radiation," Proc. IEEE, vol. 67, no. 11, pp. 1474-1486, Nov. 1979.

[27] T. Komine and M. Nakagawa, "Fundamental analysis for visiblelight communication system using LED lights," IEEE Trans. Consum. Electron., vol. 50, no. 1, pp. 100-107, Feb. 2004.

[28] J. M. Kahn and J. R. Barry, "Wireless infrared communications," Proc. IEEE, vol. 85, no. 2, pp. 265-298, Feb. 1997.

[29] X. Qiu and K. Chawla, "On the performance of adaptive modulation in cellular systems," IEEE Trans. Commun., vol. 47, no. 6, pp. 884-895, Jun. 1999.

[30] C.-S. Chang, "Stability, queue length, delay of deterministic and stochastic queueing networks," IEEE Trans. Autom. Control, vol. 39, no. 5, pp. 913-931, May 1994.

[31] C.-S. Chang and J. A. Thomas, "Effective bandwidth in high-speed digital networks," IEEE J. Sel. Areas Commun., vol. 13, no. 6, pp. 1091-1100, Aug. 1995.

[32] J. G. Kim and M. Krunz, "Bandwidh allocation in wireless networks with guaranteed packet-loss performance," IEEE/ACM Trans. Netw., vol. 8, no. 3, pp. 337-349, Jun. 2000.

[33] J. Tang and X. Zhang, "Quality-of-service driven power and rate adaptation for multichannel communications over wireless links," IEEE Trans. Wireless Commun., vol. 6, no. 12, pp. 4349-4360, Dec. 2007.

[34] I. S. Gradshteyn and I. M. Ryzhik, Table of Integrals, Series, Products. Amsterdam, The Netherlands: Elsevier, 2007.

[35] D. Bertsekas and R. Gallager, Data Networks. Upper Saddle River, NJ, USA: Prentice-Hall, 1987.

[36] F. P. Kelly, "Charging and rate control for elastic traffic," Eur. Trans. Telecommun., vol. 8, no. 1, pp. 33-37, Jan. 1997.

[37] P. Bonami et al., "An algorithmic framework for convex mixed integer nonlinear programs," Discret Optim., vol. 5, no. 2, pp. 186-204, May 2008.

[38] D. P. Palomar and M. Chiang, "A tutorial on decomposition methods for network utility maximization," IEEE J. Sel. Areas Commun., vol. 24, no. 8, pp. 1439-1451, Aug. 2006.

[39] W. Yu and R. Lui, "Dual methods for nonconvex spectrum optimization of multicarrier systems," IEEE Trans. Commun., vol. 54, no. 7, pp. 13101322, Jul. 2006.

[40] J. M. Steele, The Cauchy-Schwarz Master Class. Washington, DC, USA: Math. Assoc. Amer., 2004.

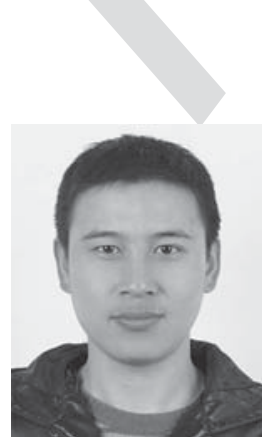

Fan Jin received the B.Eng. and M.Sc. degrees in electronics and information engineering from Huazhong University of Science and Technology, Wuhan, China, in 2008 and 2010, respectively. He is currently working toward the Ph.D. degree with the Communications Group, School of Electronics and Computer Science, University of Southampton, Southampton, U.K.

His research interests include multi-user communications, visible light communication systems, radio resource allocation, spectrum sensing, and interference management in femtocells and heterogeneous networks. He was a recipient of a scholarship under the U.K.-China Scholarships for Excellence Programme. 


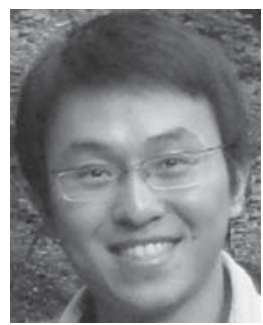

Rong Zhang (M'09) received the B.Sc. degree from Southeast University, Nanjing, China, in 2003 and the $\mathrm{Ph} . \mathrm{D}$. degree from the University of Southampton, Southampton, U.K., in 2009. Before obtaining the doctorate, he was an Engineer (August 2003-July 2004) at China Telecom and a Research Assistant (January 2006-May 2009) at Mobile Virtual Center of Excellence (MVCE), U.K. After being a Postdoctoral Researcher (August 2009-July 2012) at the University of Southampton, he took an industrial consulting leave (August 2012-January 2013) for Huawei Sweden R\&D as a System Algorithms Specialist. Since February 2013, he is a Lecturer with the CSPC Group, School of Electronics and Computer Science, University of Southampton. He has more than 40 journals in prestigious publication avenues (e.g., IEEE and OSA) and many more in major conference proceedings. He regularly serves as a Reviewer for IEEE transactions and journals and has served several times as a TPC Member/Invited Session Chair of major conferences. He was the recipient of joint funding from MVCE and EPSRC and is also a Visiting Researcher under the Worldwide University Network (WUN). More details can be found at http://www.ecs. soton.ac.uk/people/rz.

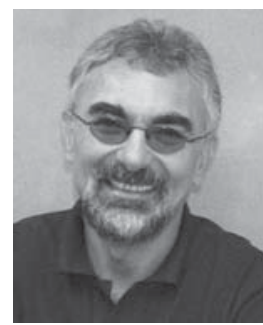

Lajos Hanzo(M'91-SM'92-F'04) received the M.S. degree in electronics and the Ph.D. degree from the Technical University of Budapest, Budapest, Hungary, in 1976 and 1983, respectively; the D.Sc. degree from the University of Southampton, Southampton, U.K., in 2004; and the "Doctor Honoris Causa" degree from the Technical University of Budapest in 2009. During his 35-year career in telecommunications, he has held various research and academic posts in Hungary, Germany, and the U.K. Since 1986, he has been with the School of Electronics and Computer Science, University of Southampton, where he holds the Chair in Telecommunications. He is currently directing a 100-strong academic research team, working on a range of research projects in the field of wireless multimedia communications sponsored by industry, the Engineering and Physical Sciences Research Council U.K., the European IST Programme, and the Mobile Virtual Centre of Excellence, U.K. During 2008-2012, he was a Chaired Professor with Tsinghua University, Beijing China. He is an enthusiastic supporter of industrial and academic liaison and offers a range of industrial courses. He has successfully supervised more than $80 \mathrm{Ph} . D$. students, coauthored 20 John Wiley/IEEE Press books on mobile radio communications totaling in excess of 10000 pages, and published 1300 research entries at IEEE Xplore. His research is funded by the European Research Council's Senior Research Fellow Grant. Dr. Hanzo is a Fellow of the Royal Academy of Engineering, the Institution of Engineering and Technology, and the European Association for Signal Processing. He is also a Governor of the IEEE Vehicular Technology Society. During 2008-2012, he was the Editor-in-Chief of the IEEE Press. He has served as the Technical Program Committee Chair and the General Chair of IEEE conferences, has presented keynote lectures, and has been awarded a number of distinctions. For further information on research in progress and associated publications, please refer to http://www-mobile. ecs.soton.ac.uk. 